\title{
Hepatic mTORC1 signaling activates ATF4 as part of its metabolic response to feeding and insulin
}

Vanessa Byles ${ }^{1}$, Yann Cormerais ${ }^{1}$, Krystle Kalafut ${ }^{1}$, Victor Barrera ${ }^{2}$, James E. Hughes Hallett ${ }^{1}$, Shannan Ho Sui ${ }^{2}$, John M. Asara ${ }^{3}$, Christopher M. Adams ${ }^{4}$, Gerta Hoxhaj ${ }^{1,5}$, Issam Ben-Sahra ${ }^{6}$, and Brendan D. Manning ${ }^{1,7}$

${ }^{1}$ Department of Molecular Metabolism, Harvard T.H. Chan School of Public Health, Boston, MA, USA

${ }^{2}$ Department of Biostatistics, Harvard T.H. Chan School of Public Health, Boston, MA, USA

${ }^{3}$ Division of Signal Transduction, Beth Israel Deaconess Medical Center and Department of Medicine, Harvard Medical School, Boston, MA, USA

${ }^{4}$ Division of Endocrinology, Metabolism and Nutrition, Mayo Clinic, Rochester, MN, USA

${ }^{5}$ Children's Medical Center Research Institute, University of Texas Southwestern Medical Center, Dallas, TX, USA

${ }^{6}$ Department of Biochemistry and Molecular Genetics, Northwestern University Feinberg School of Medicine and Robert H. Lurie Comprehensive Cancer Center, Northwestern University, Chicago, IL, USA

${ }^{7}$ Correspondence to: bmanning@hsph.harvard.edu

Disclosure of Interest: BDM is a shareholder and scientific advisory board member of Navitor Pharmaceuticals. CMA is a shareholder, director and officer of Emmyon, Inc. All other authors declare no competing financial interests.

Keywords: mTORC1, ATF4, liver, feeding, insulin, methionine metabolism 


\begin{abstract}
Objective: The mechanistic target of rapamycin complex 1 (mTORC1) is dynamically regulated by fasting and feeding cycles in the liver to promote protein and lipid synthesis while suppressing autophagy. However, beyond these functions, the metabolic response of the liver to feeding and insulin signaling orchestrated by mTORC1 remains poorly defined. Here, we determine whether ATF4, a stress responsive transcription factor recently found to be independently regulated by $\mathrm{mTORC} 1$ signaling in proliferating cells, is responsive to hepatic mTORC1 signaling to alter hepatocyte metabolism.
\end{abstract}

Methods: ATF4 protein levels and expression of canonical gene targets were analyzed in the liver following fasting and physiological feeding in the presence or absence of the mTORC1 inhibitor rapamycin. Primary hepatocytes from wild-type or liver-specific Atf4 knockout ( LAtf4 ${ }^{K O}$ ) mice were used to characterize the effects of insulin-stimulated mTORC1-ATF4 function on hepatocyte gene expression and metabolism. Both unbiased steady-state metabolomics and stable-isotope tracing methods were employed to define mTORC1 and ATF4-dependent metabolic changes. RNA-sequencing was used to determine global changes in feeding-induced transcripts in the livers of wild-type versus $L A t f 4^{K O}$ mice.

Results: We demonstrate that ATF4 and its metabolic gene targets are stimulated by mTORC1 signaling in the liver in response to feeding and in a hepatocyte-intrinsic manner by insulin. While we demonstrate that de novo purine and pyrimidine synthesis is stimulated by insulin through mTORC1 signaling in primary hepatocytes, this regulation was independent of ATF4. Metabolomics and metabolite tracing studies revealed that insulin-mTORC1-ATF4 signaling stimulates pathways of non-essential amino acid synthesis in primary hepatocytes, including those of alanine, aspartate, methionine, and cysteine, but not serine. 
Conclusion: The results demonstrate that ATF4 is a novel metabolic effector of mTORC1 in liver, extending the molecular consequences of feeding and insulin-induced mTORC1 signaling in this key metabolic tissue to the control of amino acid metabolism. 


\section{Introduction}

The liver is a central effector of systemic metabolic flexibility with a critical role in coupling shifts in glucose, lipid and amino acid metabolism to nutrient fluctuations that occur with fasting and feeding [1]. At the molecular level, the mechanistic target of rapamycin complex 1 (mTORC1) is at the heart of a nutrient sensing network that integrates nutrient availability with hormonal signals to calibrate cellular metabolism [2]. Such signal integration by mTORC1 occurs through two convergent G-protein switches, the amino acid-sensing pathway regulating the Rag GTPases and hormonal regulation of the tuberous sclerosis complex (TSC) protein complex and Rheb GTPase [3]. In the liver, mTORC1 activity is suppressed with fasting and activated with feeding to coordinate the shift between energy-producing catabolic processes and energy-consuming anabolic processes accompanying these two states [4, 5]. Feeding-induced activation of hepatic mTORC1 stimulates protein and lipid synthesis while suppressing autophagy $[4,6-8]$. Conversely, fasting diminishes mTORC1 activity in the liver, relieving its inhibitory signals on fatty acid oxidation, ketogenesis, and autophagy [5,7]. However, our current understanding of the metabolic effectors and processes downstream of mTORC1 signaling in the liver is incomplete.

Through studies largely performed in cell culture models, the mTORC1 protein kinase complex has been established to exert acute metabolic control through phosphorylation events on a growing list of direct downstream substrates. These mTORC1 targets include the canonical targets S6K1 and 4EBP1 to promote protein synthesis [9] and ULK1 and TFEB to suppress autophagy and lysosome biogenesis [10], as well as the S6K1-specific target CAD to stimulate de novo pyrimidine synthesis [11, 12]. Additionally, mTORC1 promotes metabolic alterations by engaging a downstream transcriptional network of genes encoding key metabolic enzymes [3]. Over the last decade, mTORC1 signaling has been found to stimulate a coordinated transcriptional response through regulation of specific transcription factors, including HIF $1 \alpha$ to promote glucose uptake and glycolysis [13-16], SREBP1 and 2 to stimulate lipogenesis [4, 6, 15, 
$17,18]$, and NFE2L1/NRF1 to support proteasome synthesis [19]. The regulation of these transcription factors downstream of $\mathrm{mTORC} 1$ has largely been characterized in proliferating cells in response to growth factors or oncogenic signaling. Aside from SREBP1c activation [4, 6, 20], the transcriptional effectors contributing to the metabolic response orchestrated by mTORC1 in the liver and terminally differentiated hepatocytes in response to feeding and insulin are poorly defined.

Activating transcription factor 4 (ATF4) is the best-characterized downstream effector of the integrated stress response (ISR), which is coordinated by four stress-sensing kinases - GCN2, PERK, HRI, and PKR - that converge to phosphorylate the translation initiation factor elF2 $\alpha$ at serine 51 [21]. Phosphorylation of elF2 $\alpha$ results in global attenuation of cap-dependent translation along with selective translation of ATF4, mediated by altered regulation of translation of upstream open reading frames (UORFs) in the 5' untranslated region (UTR) of the ATF4 transcript [21]. Elevated levels of ATF4 directly stimulate the expression of genes involved in adaptation to the cellular stresses initiating the ISR [22]. Beyond its canonical role in the ISR, ATF4 has also been found to be activated by anabolic signals, including insulin, downstream of mTORC1 in cell-based studies [23-26]. Pro-growth signals that activate mTORC1 stimulate ATF4 translation independently of the ISR to induce a subset of its gene targets, thereby promoting specific mTORC1-stimulated metabolic processes, including the synthesis of protein, purine nucleotides, and glutathione [24, 26]. However, our knowledge of ATF4 function in the liver is limited, and it is unknown whether physiological activation of mTORC1 promotes ATF4 function to alter hepatocyte metabolism.

In the current study, we find that physiological activation of mTORC1 by feeding in the liver and insulin in primary hepatocytes stimulates the activation of ATF4 and increased expression of ATF4-dependent gene targets. We find that despite robust mTORC1-ATF4-mediated regulation 
of previously established anabolic targets involved in serine synthesis and one carbon metabolism in response to insulin that ATF4 is dispensable for mTORC1-stimulated de novo nucleotide synthesis in primary hepatocytes. Furthermore, our study reveals that cultured primary hepatocytes synthesize little if any serine, even under serine/glycine deprivation conditions. Unbiased metabolite profiling and subsequent stable isotope-tracing experiments revealed that insulin-mTORC1-ATF4 signaling induces the synthesis of S-adenosylmethionine (SAM) in the methionine cycle and cystathionine in the trans-sulfuration pathway in primary hepatocytes. In addition, we find that insulin signaling through mTORC1 and ATF4 induces synthesis of the non-essential amino acids aspartate and alanine, albeit without detectable changes to expression of the specific transaminases involved. RNA-seq analyses of livers from control $\left(A t f 4^{f / f f}\right)$ and liver-specific Atf4 knockout $\left(L A t f 4^{K O}\right)$ mice revealed that a subset of ATF4 target genes, largely related to amino acid metabolism, were controlled by ATF4 in response to feeding. Thus, our study demonstrates that ATF4 is a novel downstream effector of physiological mTORC1 activation in the liver that contributes to the broader anabolic cellular program downstream of insulin and mTORC1 signaling.

\section{Materials and Methods}

\subsection{Mice and Diets}

All mice were maintained at the Harvard T.H. Chan School of Public Health and procedures were performed with prior approval and in accordance with the guidelines set forth by the Harvard Institutional Animal Care and Use Committee. Wild-type C57/BL6J mice (males age 68w) were purchased from Jackson Laboratories. Atf4 $4^{f / f l}$ mice were generated as previously described by the insertion of loxP sites flanking exons 2 and 3 and backcrossed to a C57/BL6J background for 9 generations [27]. Atf4//fl mice were crossed with C57/BL6J mice expressing the Albumin-Cre transgene (Jackson Labs) to generate liver-specific ATF4 knockout mice $\left(L A t f 4^{K g}\right)$, as previously described [28]. For fasting/feeding studies, mice were fasted for $12 \mathrm{~h}$ 
during the light cycle and either euthanized or fed a high carbohydrate diet (Harlan Teklad, Basal Mix adjusted for fat, TD.88122) for 6-12h in the dark cycle, as previously described[29]. Vehicle (5\% Tween-80, 5\% PEG-400, DMSO in 1x PBS) or $10 \mathrm{mg} / \mathrm{kg}$ rapamycin (LC laboratories) was injected i.p. 30 minutes prior to feeding. For all studies, mice were anesthetized with isoflurane, and when necessary, blood was collected retro-orbitally in EDTAcoated microtubes for plasma isolation. Animals were humanely euthanized, and organs were snap-frozen in liquid nitrogen, with the left lobe of the liver used for all assays. For tunicamycin treatment, mice were either injected i.p. with vehicle (150mM dextrose in 1x PBS) or tunicamycin at $1 \mathrm{mg} / \mathrm{kg}$ for $6 \mathrm{~h}$.

For glucose tolerance tests, $A t f 4^{f / f l}$ and $L A t f 4^{K O}$ male mice were fasted for $16 \mathrm{~h}$ overnight $(7 \mathrm{p} . \mathrm{m} .-$ 11a.m) and injected with glucose at $1 \mathrm{mg} / \mathrm{kg}$. For insulin tolerance tests, mice were fasted for $6 \mathrm{~h}$ during the day (8a.m.-2p.m.) and injected with insulin 0.75U/kg (Eli Lily HumulinR) dissolved in PBS plus protease free BSA. Blood glucose was monitored over time using the OneTouch® Ultra glucometer.

\subsection{Primary Hepatocyte Isolation and Culture}

Primary wild-type hepatocytes were isolated from C57/BL6J male and female mice at 7-10 weeks of age (Jackson Labs). Portal vein perfusion of buffer A (10mM HEPES, $150 \mathrm{mM} \mathrm{NaCl}$, $5 \mathrm{mM} \mathrm{KCl}, 5 \mathrm{mM}$ glucose, and 2.5mM sodium bicarbonate, 0.5mM EDTA, pH 8.0) was performed for 5-7 minutes at a rate of $5 \mathrm{ml} /$ minute followed by buffer $B$ (buffer A minus EDTA, with $35 \mathrm{mM}$ $\mathrm{CaCl}_{2}$ and Liberase TM, Sigma-Aldrich, 5401127001) for 5-7 minutes at a rate of $5 \mathrm{ml} / \mathrm{minute}$. Livers were placed in Dulbecco's Modified Eagle Medium (DMEM 4.5g/L glucose, w/o sodium pyruvate; Corning, 15-017-CV), 2.5\% FBS, Pen/Strep, and Glutamax (ThermoFisher, 35050061) or Glutagro (Corning, 25-015-CI), and the liver capsule was disrupted to release hepatocytes. Hepatocytes were centrifuged at 1000rpm for 5 minutes and then resuspended in 
10ml DMEM, 9ml Percoll (Sigma-Aldrich, P4937), and 1ml 10x PBS. Following a spin at 1000rpm for 7 minutes, hepatocytes were washed once with media, spun at 1000rpm for 5 minutes, and then resuspended in $10 \mathrm{ml}$ media per mouse. Hepatocytes from 3-5 mice were pooled and viable hepatocytes, determined by Trypan blue exclusion and cell counts, were plated at $1.25-1.5 \times 10^{6}$ cells/well in 6-well collagen-coated dishes (BioCoat, Corning) or 2.5×10 cells/6cm dishes for metabolite profiling or tracing experiments (BioCoat, Corning). After 4-6h, media was changed to serum-free media (DMEM, 2mM L-glutamine) overnight followed by insulin stimulation (100nM human insulin, Sigma-Aldrich, 19278), with 30-minute vehicle, rapamycin, or Torin1 pre-treatment, where indicated. For siRNA delivery, hepatocytes were transfected with 25-125nM siRNA using Lipofectamine RNAiMax (5 $\mu$ l 6-well, $10 \mu \mathrm{l} 6 \mathrm{~cm}$ ) 3h after plating. After $4 \mathrm{~h}$, the media was changed to serum-free media overnight or to low serum media (DMEM, 1\% FBS, 2mM L-glutamine). For siRNA experiments extending beyond 24h (Eif4ebp1 and Eif4ebp2), hepatocytes were maintained in 1nM dexamethasone with $1 \%$ FBS until incubation in serum-free media. For adenoviral delivery, hepatocytes were infected with Ad5CMV-eGFP (U. lowa Vector Core) or mouse Ad5-CMV-ATF4-eGFP (Vector Biolabs, ADV$253208) 4 \mathrm{~h}$ after plating at an $\mathrm{MOI}$ of 10 . After overnight incubation ( $16 \mathrm{~h})$, the media was changed to low serum media (1\% FBS) and incubated for another $8 \mathrm{~h}$ (total= $\sim 24 \mathrm{~h}$ ).

Primary human hepatocytes were obtained from Lonza (Cat\#HUCPG, Lot\#HUM4252) and were thawed according to the manufacturer's instructions into William's E Medium (without phenol red, ThermoFisher), 5\% FBS, Pen/Strep, 15mM HEPES, and 100nM dexamethasone and plated in 24-well dishes for RNA $(375,000 /$ well) and 12-well dishes for protein $(750,000)$. After $6 \mathrm{~h}$ of plating, hepatocytes were serum starved in William's E Medium, 10nM dexamethasone overnight. Media was changed the following morning to serum free William's E Medium (without dexamethasone) for treatment with inhibitors and 100nM insulin for $6 \mathrm{~h}$. 


\subsection{Cell Lines}

Hepa1-6 and AML-12 murine hepatocyte cell lines were obtained from the ATCC and maintained in DMEM, 4.5g/L glucose without sodium pyruvate (Thermo Scientific) and supplemented with Pen/Strep and Glutagro (Corning) and DMEM-F12 1:1, (ThermoFisher Scientific) supplemented with Pen/Strep, Glutagro (Corning), insulin-transferrin-selenium (ThermoFisher Scientific) and dexamethasone (100nM, Sigma Aldrich), respectively.

\subsection{Reagents}

After dissolving in DMSO, tunicamycin (Sigma-Aldrich, T7765) was used at $2 \mu \mathrm{g} / \mathrm{ml}$, rapamycin (EMD Millipore, 553210) at 20nM, and Torin1 (Tocris, 4247) at 500-750nM. Control nontargeting pool (D-001810-10-50) and ONTarget Plus SMARTpool siRNAs against mouse Atf4 (L-042737-01-0020, 25nM), Mthfd2 (L-042690-01-0020, 25nM), Eif4ebp1 (L-058681-01-005, 125nM), and Eif4ebp2 (L044972-01-005, 125nM) were purchased from Horizon Discovery/Dharmacon. Lipofectamine RNAiMAX was purchased from ThermoFisher Scientific.

${ }^{15} \mathrm{~N}$-glutamine-amide (490024) and ${ }^{15} \mathrm{~N}$-glutamine-amine (486809) were purchased from SigmaAldrich. $3-{ }^{13} \mathrm{C}$-serine (CLM-1572), ${ }^{13} \mathrm{C}_{5}$-methionine (CLM-893-H), and $\mathrm{U}-{ }^{13} \mathrm{C}_{6}$-glucose (CLM1396) were purchased from Cambridge Isotope Laboratories. High glucose DMEM without cystine and methionine (21013024) was purchased from ThermoFisher Scientific. DMEM without serine and glycine (U.S. Biologicals, D-9802-01) was dissolved in water and supplemented with $4.5 \mathrm{~g} / \mathrm{L}$ glucose, sodium bicarbonate, and phenol red followed by filter sterilization.

\subsection{Immunoblotting}

Protein extracts were prepared from tissues and cells using RIPA buffer ( $50 \mathrm{mM}$ Tris- $\mathrm{Cl} \mathrm{pH} 7.4$, $150 \mathrm{mM} \mathrm{NaCl}, 1 \%$ IGEPAL, $0.5 \%$ sodium deoxycholic acid, $0.1 \%$ SDS, $1 \mathrm{mM}$ EDTA, 10mM NaF, $10 \mathrm{mM}$ sodium pyrophosphate, $1 \mathrm{mM} \beta$-glycerophosphate, and $1 \mathrm{mM}$ sodium orthovanadate, 
Sigma protease inhibitor P8340). Extracts from liver tissue were prepared by homogenizing liver pieces ( 25-50mg) in RIPA buffer with protease inhibitor, Halt Phosphatase Inhibitor Cocktail (ThermoFisher Scientific, 78420), and Phosphatase Inhibitor Cocktail I (Sigma-Aldrich) using the Red Lysis Kit (Next Advance) and Next Advance Bullet Blender (speed 8, for 3 minutes). Protein concentrations were determined using a BCA assay kit (Thermo Scientific) or a detergent compatible Bradford assay (ThermoFisher Scientific). Equal amounts of protein (1520 $\mathrm{gg}$ ) were separated by SDS-PAGE, transferred to nitrocellulose membranes, and immunoblotted with indicated primary antibodies. Primary antibodies: ATF4 (BioLegend, 693902 used in primary hepatocytes and liver tissue), MTHFD2 (Proteintech,12270-1-AP), MTHFD2 (Abcam, ab151447), PHGDH (Sigma-Aldrich, HPA021241), PSAT1 (Proteintech, 20180-1-AP), PSPH (Proteintech, 14513-1-AP), ATF4 (Proteintech, 10835-1-AP, used in liver tissue), GOT2 (Proteintech, 14800-1-AP), SREBP-1 (Santa Cruz, sc-13551), $\beta$-actin (Sigma-Aldrich A5316), $\alpha-$ Tubulin (Sigma-Aldrich, T6074), phospho (P)-S6K1 T389 (Cell Signaling Technologies (CST), 9234 used in cells), P-S6K1 T389 (CST, 97596 used in liver tissue) S6K1 (CST, 2708), ATF4 (CST, \#11815 used in cell lines), PERK (CST, 3192), P-elF2 a S51 (CST, 3597), elF2 $\alpha$ (CST, 9722), P-AKT S473 (CST, 4060), Pan-AKT (CST, 4691), P-S6 S240/44 (CST, 2215), S6 (CST, 2217), 4E-BP1 (CST, 9644), 4E-BP2 (2845), P-CAD S1859 (CST, 12662), CAD (CST, 11933), P53 (CST, 32532), CHOP (CST, 5554), GAPDH (CST, 5174) and GOT1 (CST, 34423). Secondary antibodies: anti-rabbit IgG, HRP-linked (CST, 7074), anti-mouse IgG, HRP-linked (CST 7076), anti-rat IgG, HRP-linked (CST, 7077), IRDye 800CW donkey anti-mouse lgG $(\mathrm{H}+\mathrm{L})$ (LI-COR, 926-32212) and donkey ant-rabbit lgG (H+L) (LI-COR, 925-32213). Immunoblots were developed by ECL (West Pico or Femto, Thermo Scientific) or with a LI-COR Odyssey CLx imaging system (LI-COR Biosciences). For ATF4 measurement in livers, 40-50ug of protein was separated on 4-15\% TGX Criterion gels and transferred to nitrocellulose membranes. After transfer, membranes were rinsed with 1x TBS (without Tween-20) and blocked with 5\% milk in 1x TBS (without Tween-20) for $1 \mathrm{~h}$ at room temperature. Membranes were then incubated at $4^{\circ} \mathrm{C}$ 
overnight with ATF4 antibody (BioLegend) at 1:1000. After washing with 1x TBST, anti-rat HRP secondary antibody (CST) was used at 1:2000. A list of commercially available antibodies tested in livers and cell lines is included in Supplementary Table 1.

\subsection{Gene Expression Analysis}

RNA was isolated from cells (6-well dishes) or liver pieces $(\sim 10-15 \mathrm{mg})$ using the RNeasy Plus Mini kit (Qiagen). For livers, samples were homogenized in RLT buffer and suspended in an equal volume of $50 \%$ ethanol prior to application to the RNeasy columns. RNA $(0.5-1 \mu \mathrm{g})$ was reverse transcribed using the Advanced cDNA Synthesis Kit (Bio-Rad). Skirted plates and iTaq SYBR green for qPCR were purchased from Bio-Rad. QPCR analysis was performed in biological duplicates or triplicates and with duplicate technical replicates. Analysis was performed using the CFX96 Real Time PCR Detection System. Samples were normalized to Rplp0 (36b4) for ${ }^{\Delta \triangle}$ Ct analysis using the Bio-Rad CFX96 software. Primer sequences are listed in Supplementary Table 2.

\subsection{Steady State Metabolite Profiling and Targeted Metabolic Flux Analysis}

Primary mouse hepatocytes from 3-5 mice were pooled and plated at $2.5 \times 10^{6} \mathrm{cells} / 6 \mathrm{~cm}$ dish in triplicate or quadruplicate. Hepatocytes were washed twice with media lacking the amino acid used as the tracer (Gln, Ser, or Met) and incubated in the same media containing tracer for the last 30 min $\left({ }^{15} \mathrm{~N}\right.$-glutamine-amide $\left.(2 \mathrm{mM})\right)$ or $1 \mathrm{~h}\left({ }^{15} \mathrm{~N}\right.$-glutamine-amine $(2 \mathrm{mM}), 3-{ }^{13} \mathrm{C}_{1}$-serine $(400 \mu \mathrm{M})$, or ${ }^{13} \mathrm{C}_{5}$-methionine $\left.(200 \mu \mathrm{M})\right)$ of insulin stimulation. Metabolites were extracted with $80 \%$ methanol (HPLC grade) for 15 minutes at $-80^{\circ} \mathrm{C}$, with cells subsequently scraped off of plates into $80 \%$ methanol on dry ice and placed into $15 \mathrm{ml}$ conical tubes. After 5 -minute centrifugation at maximum speed, supernatants were transferred to $50 \mathrm{ml}$ conical tubes. A second extraction was performed on the remaining pellet with $500 \mu$ of ice-cold $80 \%$ methanol, centrifuged, and pooled with the first extraction in $50 \mathrm{ml}$ conical tubes. After the final extraction, 
remaining insoluble pellets were resuspended in $500 \mu$ of $8 \mathrm{M}$ urea in $10 \mathrm{mM}$ Tris- $\mathrm{Cl} \mathrm{pH} 8.0$ and shaken at $60^{\circ} \mathrm{C}$ for $1 \mathrm{~h}$. Protein concentrations were measured for sample normalization using a BCA or Bradford assay. Metabolite extracts were dried under a stream of nitrogen gas using an N-EVAP (Organomotion Associates, Inc.).

For metabolite tracing into the nucleotides of total cellular RNA, primary hepatocytes plated in 6 well dishes were labeled with ${ }^{15} \mathrm{~N}$-glutamine-amide $(2 \mathrm{mM})$ for $6 \mathrm{~h}$. RNA was isolated using the

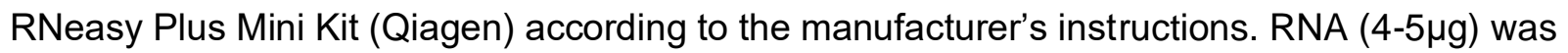
heated to $100^{\circ} \mathrm{C}$ for 3 minutes and rapidly cooled in an ice-water bath. Samples were brought up to $50 \mu \mathrm{l}$ and subsequently digested with $1 \mu \mathrm{l}$ of Nuclease $\mathrm{P} 1$ in $50 \mathrm{mM}$ sodium acetate buffer (New England Biolabs, M0660) for $2 \mathrm{~h}$ at $45^{\circ} \mathrm{C}$. Samples were then neutralized with $1 / 10$ volume of $1 \mathrm{M}$ ammonium bicarbonate (made fresh) and digested with $2 \mu \mathrm{l}$ of $0.25 \mathrm{U} / \mu \mathrm{l}$ Phosphodiesterase I from Crotalus adamanteus venom (Sigma-Aldrich, P3243) for $2 \mathrm{~h}$ at $37^{\circ} \mathrm{C}$. Samples were then extracted in $80 \%$ methanol and dried under nitrogen gas using an N-EVAP.

Dried-down metabolites were re-suspended in $30 \mu \mathrm{L}$ of HPLC-grade water and $5 \mu \mathrm{L}$ of sample was injected for liquid chromatography/mass spectrometry (LC/MS) using a 6500 QTRAP hybrid triple quadrupole mass spectrometer (AB/SCIEX) coupled to a Prominence UFLC HPLC system (Shimadzu) with Amide XBridge HILIC chromatography (Waters) via selected reaction monitoring (SRM, Supplementary Table 3) and polarity switching between positive and negative modes [30]. For steady state profiling, selected reaction monitoring of a total of 254 polar metabolites was analyzed [30]. Parameters for software analysis were as previously described [11]. Peak areas from the total ion current for each metabolite SRM transition were integrated using MultiQuant v2.0 software (AB/SCIEX). Peak areas were normalized to protein levels from insoluble pellets obtained during the metabolite extraction. As a control, an unlabeled sample 
was run in parallel to account for natural abundance for comparison to $m+1$ labeled isotopologues. For RNA tracing experiments, the unlabeled signal was subtracted from the labeled isotopologues before calculating the fractional enrichment. For steady state metabolomic profiling, KEGG enrichment analysis was performed using MetaboAnalyst software and the heatmap of significantly altered metabolites was generated using Morpheus software (Broad Institute of MIT and Harvard).

\subsection{RNA-Sequencing}

RNA was isolated from the livers of $A t f 4^{f / f l}$ and $L A t f 4^{K O}$ mice ( $\left.n=5 / g r o u p\right)$ with the Qiagen RNeasy Mini kit. RNA-sequencing was performed at the Dana-Farber Cancer Institute Sequencing Core. Strand-specific libraries were generated with 500ng of RNA using TruSeq library preparation kit (Illumina, San Diego, CA). The cDNA libraries were multiplexed and sequenced using Illumina NextSeq 500 with single-end 75bp read length parameters. Adapter sequences were trimmed off sequencing adaptors and low-quality regions by using cutadapt [31]. Trimmed reads were aligned to UCSC build mm10 of the Mus musculus genome, using STAR [32]. After the counts were collected, differential gene expression analysis was performed using DE-Seq2, which calculated fold change and adjusted p-values [33]. Lists of differentially expressed genes were examined for gene ontology (GO) and KEGG term enrichment with clusterProfiler [34]. Gene set enrichment analysis was performed using MSigDB annotated gene sets (Broad Institute of MIT and Harvard). The complete RNA-seq data can be found at GEO under the accession number GSE173578.

\subsection{Statistical Analysis}

All statistical analyses were performed using GraphPad Prism 8.0 using either Student's t-tests, One-way ANOVA (with Holm-Sidak post-hoc analysis) or Two-way ANOVA (with Holm-Sidak 
pos-hoc analysis), as indicated in each figure legend. ${ }^{*} p<0.05,{ }^{* *} p<0.01,{ }^{* *} p<0.001$, ${ }^{* * * *} p<0.0001$.

\subsection{Immunoblot quantification and schematic generation.}

Western blots were quantified using either LICOR or ImageJ software. Schematic figures were generated using BioRender software.

\section{Results}

\subsection{Physiological stimulation of mTORC1 signaling activates ATF4 and expression of established gene targets.}

To determine if mTORC1 activation in the liver stimulates an increase in ATF4 protein with physiological feeding, mice were subjected to a $12 \mathrm{~h}$ light-cycle fast followed by $6 \mathrm{~h}$ refeeding in the dark cycle with a high carbohydrate diet. As expected, refeeding potently stimulated mTORC1 activity as assessed by phosphorylation of its downstream targets S6K1 and 4E-BP1, the latter reflected in an upward electrophoretic mobility shift (Figure 1A). Additionally, we observed a feeding-induced, rapamycin-sensitive increase in phosphorylation of the S6K1 target CAD, the first enzyme of de novo pyrimidine synthesis $[11,12]$, as well as the namesake S6K1 substrate ribosomal protein S6. Importantly, feeding-induced mTORC1 signaling also stimulated increased protein expression of ATF4, which could be blunted by administration of rapamycin prior to feeding. Refeeding mice $12 \mathrm{~h}$ in the dark cycle also led to induction of liver ATF4 protein levels in an mTORC1-dependent manner with overall reductions in mouse to mouse variability (Figure S1A). Importantly, unlike ATF4, elF2 $\alpha$ phosphorylation in the liver, indicative of ISR induction, was not consistently affected by fasting, feeding, or rapamycin, supporting previous studies in cell-based models that mTORC1 can activate ATF4 occurs independent of the ISR $[24,26]$. As we had found previously that mTORC1 activation stimulates expression of genes encoding enzymes of the serine synthesis pathway (Phgdh, Psat, Psph) 
and mitochondrial one-carbon metabolism (Mthfd2) through ATF4 [24], we assessed whether these genes were influenced by feeding and mTORC1 signaling in the liver. Indeed, along with Atf4 transcripts, feeding induced expression of these genes in a rapamycin-sensitive manner (Figure 1B). However, rapamycin did not affect the ability of feeding to suppress expression of the gluconeogenic gene Pepck, indicative of a lack of global effects from this treatment on the feeding response.

Given that the liver contains both parenchymal and non-parenchymal cells, we sought to characterize the cell-intrinsic regulation of ATF4 and its transcriptional targets by mTORC1 in primary mouse hepatocytes. Over a time course of insulin stimulation, where Akt is fully activated within 5 min and mTORC1 signaling by 30 min, and increase in ATF4 protein levels was evident by $30 \mathrm{~min}$, peaking at 2 hours (Figure 1C). Insulin-stimulated mTORC1 signaling and induction of ATF4 were both blocked by rapamycin or the kinase domain inhibitor of mTOR Torin1, which has more potent suppressive effects on mTORC1 targets and global protein synthesis [35]. Additionally, while there was no observable change in elF2a phosphorylation with insulin or rapamycin treatment, we did observe that Torin1 abrogated the basal elF2 $\alpha$ phosphorylation signal with time (Figure 1C), suggesting that the stronger effects of Torin1 on ATF4 and its gene targets observed (see below) might be due to combined effects on both mTORC1 signaling and the ISR. For this reason, in all subsequent experiments, we use rapamycin instead of, or in parallel to, Torin1 treatment, to focus on mTORC1-specific effects on ATF4 regulation and function.

We next sought to determine how mTORC1 signaling stimulates hepatic ATF4 activation in response to insulin. A previous report demonstrated that mTORC1-dependent regulation of ATF4 occurs through phosphorylation and inactivation of the translational repressors 4E-BP1 and 4E-BP2 $[25,36]$. Indeed, silencing of 4E-BP1 and 4E-BP2 in primary hepatocytes with 
siRNAs resulted in elevated basal ATF4 protein levels and resistance to rapamycin and Torin1 treatment relative to hepatocytes transfected with control siRNAs (Figure 1D). Interestingly, while there was no effect of rapamycin on elF2 $\alpha$ phosphorylation under either condition, knockdown of 4E-BP1/2 rescued the effects of Torin1 treatment on basal elF2 $\alpha$ phosphorylation, indicating that these effects are likely linked to the 4EBP1/2-dependent attenuation of protein synthesis reported with Torin1 treatment in other settings [35], which could increase amino acid availability and minimize protein load on the $E R$, thus decreasing basal activation of the ISR.

To further characterize the hepatocyte-intrinsic effects of the mTORC1-ATF4 axis, we performed insulin time course experiments analyzing the transcript and protein levels of the aforementioned ATF4 targets. Based on time course experiments, the peak of ATF4 protein production upon insulin stimulation was at $2 \mathrm{~h}$, with levels steadily declining over the time course up to 8 h (Figure 1C, E). Induction of Mthfd2, Phgdh, Psat1, and Psph transcript levels was detected by $2 \mathrm{~h}$ insulin stimulation, peaking at $4 \mathrm{~h}$, while corresponding protein levels were robustly induced by 6h (Figure 1E, F, with protein quantified in Figure S1B). Importantly, both mRNA and protein levels of these gene targets were sensitive to rapamycin and Torin1, with the latter demonstrating more potent effects accompanying decreased elF2 $\alpha$ phosphorylation. In addition to primary hepatocytes, the commonly used murine hepatocyte cell lines, Hepa1-6 and AML-12, also displayed insulin-stimulated increases in ATF4 and expression of these gene targets that was blocked by mTOR inhibitors (Figure S1C-D). Finally, in primary human hepatocytes, insulin induced ATF4 protein in an mTORC1-dependent manner, without effects on elF2 $\alpha$ phosphorylation (Figure 1G), and this regulation correlated with stimulated expression of MTHFD2, PSAT1, and PSPH transcripts (Figure 1H). Together, these findings 
define ATF4 as a novel downstream target of physiological mTORC1 activation with feeding and insulin in the liver and cell-autonomously in hepatocytes.

\subsection{ATF4 is required for insulin-mTORC1 signaling to induce the expression of serine synthesis and one carbon metabolism enzymes in hepatocytes.}

Both the basal and insulin-stimulated expression of MTHFD2, PHGDH, PSAT1, and PSPH transcripts and proteins were abrogated in primary hepatocytes with siRNA-mediated knockdown of Atf4 (Figure 2A,B). As an orthogonal approach, we generated mice with liverspecific deletion of Atf4 ( $L A t f 4^{K O}$ ), using previously described Atf4 $4^{f / f l}$ mice crossed to mice expressing the Cre recombinase from the liver-specific albumin promoter $[27,28]$. In primary hepatocytes isolated from control Atf4 $4^{f / f l}$ mice, insulin induced MTHFD2, PHGDH, PSAT1, and PSPH protein and transcripts in a rapamycin-sensitive manner, but this regulation was greatly reduced in the $L A t f 4^{K O}$ hepatocytes (Figure 2C,D). Of note, the LAtf4 ${ }^{K O}$ hepatocytes, like those with siRNA knockdown of Atf4, displayed enhanced insulin-stimulated mTORC1 signaling, perhaps indicative of loss of ATF4-dependent targets that negatively regulate mTORC1 [37-40]. To confirm the specificity of the ATF4-dependent responses, primary hepatocytes from LAtf4 ${ }^{K O}$ mice were infected with adenoviruses expressing either GFP-encoding control (AdGFP) or an ATF4 cDNA (AdATF4). The markedly diminished expression of Mthfd2, Phgdh, Psat1, and Psph transcripts in the LAtf4 ${ }^{K O}$ hepatocytes was fully restored with AdATF4. Furthermore, ectopic expression of ATF4 in wild-type primary hepatocytes resulted in substantial overexpression of ATF4 that correlated with enhanced MTHFD2 and PSAT1 protein levels (Figure 2G). As the AdATF4 adenovirus lacks the endogenous 5'UTR of ATF4, which is required for its regulation by mTORC1 [24-26], the enhanced expression of ATF4, MTHFD2 and PSAT1 induced by AdATF4 was resistant to rapamycin treatment. Together, these results establish that the mTORC1-ATF4 axis regulates these gene targets in primary hepatocytes with potential 
implications for metabolic control of serine and purine nucleotide synthesis, as observed in proliferative settings [24].

\section{3. mTORC1 stimulates hepatocyte nucleotide synthesis in an ATF4-independent}

manner

In proliferating cells, mTORC1 regulates de novo purine nucleotide synthesis, in part, through ATF4-dependent regulation of MTHFD2 and the mitochondrial tetrahydrofolate cycle, which supplies one carbon formyl units derived from the carbon 3 atom of serine to the purine ring [24]

(Figure 3A). While primary hepatocytes are terminally differentiated cells with minimal DNA synthesis, they still have a high demand for nucleotides to produce rRNA needed for ribosome biogenesis in this secretory organ. Indeed, we found that insulin stimulated an mTORC1dependent increased flux through the de novo purine synthesis pathway in primary hepatocytes, as measured by ${ }^{15} \mathrm{~N}$-glutamine-amide tracing into the newly synthesized free pool of cellular purine nucleotides, including IMP $(m+2)$, ATP $(m+2)$ and GTP $(m+3)$, the latter of which acquires a third ${ }^{15} \mathrm{~N}$ atom from the glutamine amide (Figure $3 \mathrm{~B}$ ). Likewise, ${ }^{-1{ }^{13}} \mathrm{C}_{1}$-serine tracing, which specifically measures incorporation of the two formyl units into the purine ring, also revealed that insulin stimulates increased de novo purine synthesis in an mTORC1-dependent manner (Figure S2A). We next determined the effects of insulin-mTORC1 signaling on flux through de novo purine synthesis into the total RNA pool of primary mouse hepatocytes, of which approximately $80 \%$ represents rRNA [41]. The fractional enrichment for RNA-derived purines labeled with ${ }^{15} \mathrm{~N}$-glutamine-amide via de novo synthesis during an 8 h stimulation with insulin was surprisingly low (<4\%). However, an insulin-stimulated, mTORC1-dependent increase in the fractional enrichment of de novo synthesized AMP $(m+2)$ and GMP $(m+3)$ within RNA was observed (Figure 3C). Unexpectedly, siRNA-mediated knockdown of Atf4 in primary hepatocytes did not impair the insulin-stimulated increase in ${ }^{15} \mathrm{~N}$-glutamine-amide or $3-{ }^{13} \mathrm{C}_{1}$ serine flux into free pools of purine nucleotides (Figure 3D and Figure S2B). Furthermore, 
direct silencing of Mthfd2 also failed to impact the ability of insulin to stimulate de novo purine synthesis (Figure S2B). Likewise, Atf4 knockdown had only minor impact on the fractional enrichment of de novo synthesized AMP $(m+2)$ and GMP $(m+3)$ derived from total hepatocyte RNA (Figure 3E).

The effects of insulin-mTORC1 signaling on de novo synthesis of pyrimidine nucleotides was also assessed in primary hepatocytes. Consistent with the stimulated phosphorylation and activation of CAD by S6K1 downstream of mTORC1 detected in liver and primary hepatocytes

(Figure 1A,E) [11, 12], insulin stimulated ${ }^{15} \mathrm{~N}$-glutamine-amide flux into free pools of UTP $(m+1)$ and CTP $(m+2)$ and RNA-derived UMP $(m+1)$ and CMP $(m+2)$ in an mTORC1-dependent manner (Figure 3F,G and Figure S2C). Based on the role of ATF4 in regulating non-essential amino acid metabolism in other cellular settings [22, 25, 26, 42], we assessed de novo pyrimidine synthesis upon Atf4 knockdown. Rather than inhibiting insulin-stimulated pyrimidine synthesis, loss of ATF4 moderately enhanced ${ }^{15} \mathrm{~N}$-glutamine-amide flux into the free pools of pyrimidines and had no observable effects on the fractional enrichment of labeling into RNAderived pyrimidines (Figure $\mathbf{3 H}$ and Figure S2D). Thus, insulin-mTORC1 signaling induces de novo synthesis of both purine and pyrimidine nucleotides in primary hepatocytes through mechanisms independent of ATF4.

\subsection{Lack of evidence for regulated serine synthesis in primary hepatocytes}

The de novo serine synthesis pathway (SSP) is a metabolic branchpoint in glycolysis comprised of three successive steps catalyzed by the PHGDH, PSAT1, and PSPH enzymes, with the product serine contributing to multiple biosynthetic processes, in addition to protein and nucleotide synthesis (Figure 4A). To measure SSP activity in primary hepatocytes, we used

${ }^{15} \mathrm{~N}$-glutamine-amine tracing to capture the transamination of 3-phosphohydroxypyruvate to 3phosphoserine catalyzed by PSAT1 and serine generation following dephosphorylation by 
PSPH (Figure 4A). Despite robust regulation of PHGDH, PSAT1, and PSPH expression by mTORC1-ATF4 signaling, described above, insulin failed to stimulate ${ }^{15} \mathrm{~N}$-glutamine-amine flux into the free pool of labeled serine $(m+1)$ in primary hepatocytes (Figure 4B). One possibility is that the newly synthesized serine was being rapidly utilized for protein synthesis, however, treatment with the protein synthesis inhibitor cycloheximide during the $1 \mathrm{~h}$ labeling did not result in accumulation of labeled serine. Surprisingly, this $1 \mathrm{~h}$ labeling yielded a fractional enrichment of labeled serine of less than $5 \%$ in primary hepatocytes, which was unaltered by insulin, mTORC1 inhibitors, or cycloheximide (Figure 4C). This result was unchanged with labeling times from 30 min up to $6 \mathrm{~h}$, indicating that flux through the SSP is unusually low in primary hepatocytes (Figure S3A,B), even under these conditions where the expression of SSP enzymes are induced by insulin through mTORC1 and ATF4. Consistent with this disconnect between pathway enzyme regulation and SSP flux, siRNA-mediated knockdown of Atf4 had no effect on the relative abundance or fractional enrichment of labeled serine $(m+1)$ in primary hepatocytes (Figure 4D,E).

We next determined whether primary hepatocytes were capable of activating SSP flux in response to serine and glycine deprivation, as other cell types do [43-45]. 24-h serine starvation of primary hepatocytes resulted in a marked reduction in intracellular serine levels that was further reduced with combined serine and glycine starvation (Figure 4F). However, this serine depletion had little effect on the fractional enrichment of newly synthesized serine $(m+1)$ detected with ${ }^{15} \mathrm{~N}$-glutamine-amine labeling (Figure 4G). As glucose is the primary source of carbon for serine synthesis, we also utilized U- ${ }^{13} \mathrm{C}$-glucose tracing into serine. Despite robust (80\%) labeling of 3-phosphoglycerate $(m+3)$, the glycolytic intermediate precursor to the SSP, the fractional enrichment of glycolysis-derived labeled serine $(m+3)$ was only slightly increased with serine deprivation and still accounted for less than $1 \%$ of total cellular serine (Figure $\mathbf{4 H}$ ). This is in contrast to another product downstream of 3-phosphoglycerate, lactate, which was 
nearly $60 \% \mathrm{~m}+3$ labeled over the same duration. Together, these results indicate that primary hepatocytes in culture synthesize very little serine, even when the enzymes of the SSP pathway are elevated or cells are deprived of exogenous serine.

\subsection{ATF4 regulates methionine metabolism in hepatocytes}

As the above studies on nucleotide and serine synthesis revealed that hepatocytes are distinct from other cellular systems in the control of these processes, we employed unbiased steady state metabolomics to identify potential insulin-stimulated metabolic changes dependent on ATF4. This analysis revealed that several metabolites were significantly elevated $(p<0.05)$ upon insulin treatment in control hepatocytes but not those with siRNA-mediated knockdown of Atf4 (Figure 5A, with the top 6 metabolites shown graphically in Figure 5B, Supplementary Table 4). The metabolites induced most strongly with insulin in an ATF4-dependent manner included those related to the transsulfuration pathway and the methionine cycle, including cystathionine and S-adenosylmethionine (SAM) (Figure 5A-C). Indeed, enrichment analysis of the insulinATF4 regulated metabolites revealed an overrepresentation of KEGG metabolite sets for amino acid metabolism, including that of cysteine and methionine (Figure S5A). Additionally, we observed decreased levels of reduced glutathione upon ATF4 knockdown, with modestly increased levels of oxidized glutathione disulfide (GSSG) (Figure 5A, Figure S5B). We recently demonstrated that the mTORC1-ATF4 axis stimulates the production of glutathione through induction of SLC7A11 expression and increased cystine uptake in proliferating cells [26]. However, Slc7a11 expression was not regulated by insulin or ATF4 in primary hepatocytes, nor were the glutathione synthesis enzymes Gclc and Gclm (Figure S4C).

The methionine cycle produces SAM through the activity of the methionine adenosyltransferase (MAT) enzymes (MAT1 and MAT3 in liver [46]), and SAM is the methyl donor for a variety of cellular processes including histone, DNA, and protein methylation and phosphatidylcholine 
synthesis [47]. The methionine cycle is tightly coupled to both folate metabolism and the transsulfuration pathway (Figure 5C). Given the observed alterations in metabolites of the methionine cycle and transsulfuration pathway, we broadly assessed the expression of genes encoding enzymes within this metabolic network in response to insulin and Atf4 knockdown. Genes of the cytosolic THF and methionine cycles were insensitive to insulin and ATF4 depletion in primary hepatocytes (Figure 5D). Notably, the MAT enzymes were not found to be transcriptionally regulated in this setting, in contrast to proliferating cells, where MAT2A has recently been found to be regulated by mTORC1 signaling via c-Myc [48]. Interestingly, only Cth, encoding an enzyme of the transsulfuration pathway, also known as cystathionine gamma lyase (CGL), was found to be significantly induced with insulin in an ATF4-dependent manner (Figure 5D). Like other targets of the mTORC1-ATF4 axis in primary hepatocytes (Figure 1F), the insulin-stimulated expression of Cth was also found to peak at $4 \mathrm{~h}$ and be fully suppressed by rapamycin or Torin1 treatment (Figure S4D). The insulin- and mTORC1-mediated regulation of $C$ th expression was lost in primary hepatocytes derived from LAtf4 ${ }^{K O}$ mice, and Cth expression was restored to these cells with exogenous ATF4 (Figure S4E,F).

To determine if the insulin-mTORC1-ATF4 pathway influenced metabolic flux into the methionine cycle and transsulfuration pathways in primary hepatocytes, we utilized stableisotope tracing. Indeed, insulin stimulated $3-{ }^{13} \mathrm{C}_{1}$-serine tracing into labeled $\mathrm{SAM}(\mathrm{m}+1)$ and cystathionine $(m+1)$ in a manner that was attenuated by either mTOR inhibitors or siRNA knockdown of Atf4 (Figure 5E,F). Of note, no change in the labeling of other metabolites of the methionine cycle, including methionine, S-adenosylhomocysteine (SAH), and homocysteine, were detected in this experiment (data not shown). To further assess the point of regulation of insulin- and ATF4-mediated SAM synthesis, we employed ${ }^{13} \mathrm{C}_{5}$-methionine tracing to directly test involvement of methionine conversion to SAM via MAT isoforms. Robust SAM labeling $(m+5)$ was detected $(>70 \%$ fractional enrichment after $1 \mathrm{~h}$ labeling) but was unaffected in 
primary hepatocytes first stimulated with insulin or depleted of ATF4 (Figure 5G,H). Conversely, insulin stimulated an increase in labeling of the transsulfuration metabolites cystathionine $(m+4)$ and $\alpha$-ketobutyrate $(\mathrm{m}+4)$ from ${ }^{13} \mathrm{C}_{5}$-methionine in a manner blunted by Atf4 knockdown (Figure 5I and Figure S4G). Thus, insulin and ATF4 induce SAM synthesis through a mechanism independent of MAT regulation and stimulate the transsulfuration pathway downstream of the methionine cycle.

\subsection{Insulin induces hepatocyte aspartate and alanine synthesis via ATF4}

The steady state metabolomic profiling of primary hepatocytes with Atf4 knockdown also showed that the non-essential amino acids aspartate and alanine were increased with insulin in an ATF4-dependent manner (Figure 5A). To determine whether this change reflects a stimulated increase in the synthesis of these amino acids, we employed stable isotope tracing with ${ }^{15} \mathrm{~N}$-glutamine-amine labeling. Aspartate synthesis results from transamination of oxaloacetate by GOT1 in the cytosol and GOT2 in the mitochondria (Figure S5A). ${ }^{15} \mathrm{~N}$ glutamine-amine flux into labeled aspartate $(m+1)$ in primary hepatocytes was increased with insulin in a rapamycin-resistant, but Torin1-sensitive manner (Figure S5B), perhaps reflecting the more potent effects of Torin1 on ATF4 and its targets (Figure 1). One-hour treatment with cycloheximide increased the labeled pool of aspartate, indicating that a portion of newly synthesized aspartate is rapidly utilized for protein synthesis. Consistent with the steady state measurements (Figure 5A,B), stable-isotope tracing found that insulin stimulated an increase in aspartate synthesis in a manner ablated by siRNA knockdown of Atf4 (Figure S5C). However, unlike other cell types [26, 49], the expression of neither Got1 nor Got2 were affected by ATF4 depletion in hepatocytes (Figure S5D-E). We next assessed alanine synthesis in primary hepatocytes, which results from transamination of pyruvate by GPT1 in the cytosol and GPT2 in the mitochondria (Figure S5F). Much like aspartate synthesis, ${ }^{15} \mathrm{~N}$-glutamine-amine flux into labeled alanine $(m+1)$ was increased with insulin in rapamycin-resistant, but Torin1-sensitive 
manner in primary hepatocytes (Figure S6G). Of note, cycloheximide treatment had minimal effects on the free pool of newly synthesized alanine. Also similar to aspartate, stable-isotope tracing found that insulin stimulated an increase in alanine synthesis in an ATF4-dependent manner (Figure S6H). Previous studies have linked ATF4 to the transcriptional regulation of Gpt2 [26, 42, 49, 50], and Atf4 knockdown decreased hepatocyte Gpt2 expression under both basal and insulin-stimulated conditions (Figure S6H). However, unlike alanine synthesis, insulin did not stimulate a significant increase in Gpt2 transcripts, suggesting that these two observations may be unrelated. Together, these results indicate that insulin-mTORC1 signaling stimulates aspartate and alanine synthesis in primary hepatocytes via ATF4, albeit through a currently unknown mechanism.

\subsection{Feeding induces an ATF4-dependent transcriptional response in the mouse liver}

Previous studies have challenged the $L A t f 4^{K O}$ mice with stress stimuli that engage the ISR [28, 51], but there are no reports to date characterizing the transcriptional response to feeding in these mice. To first confirm that ATF4 was ablated in the livers of $L A t f 4^{K O}$ mice, and to screen commercially available antibodies for specific recognition of ATF4 in the mouse liver, we treated $A t f 4^{f l / f l}$ and $L A t f 4^{K O}$ mice with the ER stress-inducing agent tunicamycin to stimulate a robust increase in hepatic ATF4 levels (Figure S6A). Indeed, tunicamycin failed to induce detectable ATF4 protein in the livers of $L A t f 4^{K O}$ mice. It is also important to note that this analysis revealed that some widely used ATF4 antibodies recognize non-specific bands at the same molecular mass as ATF4 on immunoblots of mouse liver extracts. We next used the same daytime fasting and nighttime refeeding paradigm employed in Figure $1 A, B$. The $L A t f 4^{K O}$ livers displayed comparable levels of $\mathrm{mTORC} 1$ activation to the $A t f 4^{f / f t}$ control livers upon refeeding, which correlated with a robust increase in both full length and processed forms of SREBP1c, a known effector of hepatic mTORC1 with feeding [4, 6, 20] (Figure 6A). It is worth noting that this result is counter to what was observed previously in livers from whole body Atf4 ${ }^{-/}$mice, which were 
reported to display a decrease in hepatic SREBP1c activation, measured by expression of its transcriptional targets $[52,53]$. In addition to normal induction of mTORC1 signaling, the feeding-induced expression of the SREBP1c target Fasn and suppression of the gluconeogenic gene Pepck were similar between the $A t f 4^{f / f f l}$ and $L A t f 4^{K O}$ livers, indicative of a normal feeding response in the $L A t f 4^{K O}$ livers (Figure 6B). Gene expression analysis confirmed our previous observation that feeding induces the expression of the serine synthesis pathway genes (Phgdh, Psat1, and Psph) and Mtfhfd2 in the Atf4 ${ }^{f / f l}$ livers, and this response was blunted in the LAtf4 ${ }^{K O}$ livers, providing genetic evidence of their regulation by feeding through ATF4 activation.

Given that whole body Atf4-- mice display growth defects and lower body weights [54], we assessed physiological parameters in the LAtf4 ${ }^{K O}$ mice. We found that body weights of the $A t f 4^{f l / f l}$ and $L A t f 4^{K O}$ mice were similar at 8 weeks, however there was a small, but statistically significant, body weight reduction in the $L A t f 4^{K O}$ mice at 26 weeks of age (Figure S6B). Furthermore, there were no significant differences observed in systemic glucose and insulin tolerance between $A t f 4^{f / f t}$ and $L A t f 4^{K O}$ mice at 26 weeks of age on a chow diet (Figure S6C), consistent with previous observations [51].

To more broadly define the transcriptional output of ATF4 downstream of physiological mTORC1 activation, we performed RNA-seq analysis on Atf4//fl and LAtf4 ${ }^{K O}$ livers with fasting and refeeding. Principal component analysis of the normalized, differentially expressed transcripts revealed that transcriptional changes with fasting and feeding produce the most robust changes between groups, accounting for approximately $39 \%$ of the overall variance, whereas genotype accounted for approximately $10 \%$ of the variance (Figure 6C). We focused on the subset of genes that were significantly induced with feeding in the Atf4 ${ }^{f / f l}$ livers but not in the $L A t f 4^{K O}$ livers $\left(141\right.$ genes, $\log _{2} F C>1, p<0.05$; heatmap in Figure 6D and Supplementary Table 5). Gene set enrichment analysis revealed that previously defined ATF4 target genes 
involved in amino acid synthesis were enriched in the genes most downregulated in the LAtf4 ${ }^{K O}$ livers relative to the $A t f 4^{f / f f}$ livers with feeding (Figure 6E). These data indicate that the recently recognized role of ATF4 in amino acid metabolism downstream of mTORC1 signaling obtained from cell culture models is active in the liver in response to feeding [25, 26].

Notably, gene set enrichment analysis revealed that p53 target genes are enriched in the list of upregulated genes in $L A t f 4^{K O}$ livers relative to the $A t f 4^{f / f l}$ livers with feeding (Figure S7A). In line with this observation, $L A t f 4^{K O}$ livers from both fasted and fed mice displayed a robust increase p53 protein levels relative to the Atf4 ${ }^{f / f t}$ livers (Figure S7B). Moreover, the p53 target genes Cdkn1a (p21), Ddias, Phlda3, Psrc1, and Eda2r were all confirmed by qRT-PCR to be significantly upregulated in the LAtf4 ${ }^{K O}$ livers relative to the Atf4 $4^{f / f l}$ livers (Figure S7C). To determine if this activation of p53 was intrinsic to hepatocytes, we isolated primary hepatocytes from the livers of $A t f 4^{f / f l}$ and $L A t f 4^{K O}$ mice. Indeed, primary hepatocytes from $L A t f 4^{K O}$ mice also displayed upregulation of representative p53 target genes and p53 protein levels (Figure S7D,E). However, exogenous expression of ATF4 failed to suppress p53 or its gene targets in the $L A t f 4^{K O}$ hepatocytes, while predictably restoring expression of the canonical ATF4 target Asns (Figure S7D-E). Furthermore, the expression of p53 target genes was unaffected by siRNA-mediated knockdown of ATF4 in primary hepatocytes (Figure S7F). Thus, the strong induction of p53 and its transcriptional targets upon loss of liver ATF4 may not be an immediate or direct effect of ATF4 loss in hepatocytes.

\subsection{Secondary effects of Cre-mediated recombination at the Atf $4^{f / f l}$ locus}

Examination of the top ten most downregulated genes in the $L A t f 4^{K O}$ livers revealed that, along with established ATF4 targets (Psat, Asns, Fgf21), Rps19bp1, a previously described ribosomal protein S19-binding protein linked, among other things, to the regulation of protein synthesis [55], was second only to Atf4 among most downregulated genes (Figure 6F). Of note, siRNA- 
mediated knockdown of Rps19bp1 in primary hepatocytes did not alter the expression of p53 target genes (Figure S7F; discussed below). Five of the top ten most upregulated genes in the LAtf4 ${ }^{K O}$ livers relative to $A t f 4^{f / f f l}$ controls were established targets of $p 53$, but the most increased RNA-seq transcript reads were from the Cacna1i gene, encoding a brain-specific calcium transporter (Cav3.3) previously linked to schizophrenia [56]. Validation experiments demonstrated that Rps19bp1 expression was completely abrogated in the LAtf4 ${ }^{K O}$ livers, while Cacna $1 i$ is upregulated $\sim 400-500$ fold in the LAtf4 ${ }^{K O}$ livers relative to the control livers (Figure 6G). However, acute silencing of Atf4 with siRNAs in primary hepatocytes did not significantly alter Rps19bp1 or Cacna1i expression, suggesting that the effects are specific to the LAtf4 ${ }^{K O}$ context (Figure $6 \mathrm{H}$ ). Indeed, the gene expression findings from the liver were recapitulated in primary hepatocytes cultured from these mice, but exogenous re-expression of ATF4 in LAtf4 ${ }^{K O}$ hepatocytes failed to rescue the lost expression of Rps19bp1, while modestly but significantly reducing Cacna1i expression (Figure 6I). Examination of the Atf4 locus on mouse chromosome 15 revealed that both Rps19bp1 and Cacna1i were proximal to Atf4. The end of the Atf4 3'UTRencoding region is approximately $3 \mathrm{~kb}$ from that of the convergently transcribed Rps $19 \mathrm{bp} 1$ gene, an intergenic region where the 3' LoxP site is located in the Atf4 ${ }^{f / f l}$ allele (Figure 6J). In addition, the start codon of the Cacna1igene lies approximately $24 \mathrm{~kb}$ from that of the Rps19bp1 gene. It is worth noting that expression of genes in loci upstream of Atf4, such as Mgat3, were not perturbed by Cre-mediated recombination in the LAtf4 ${ }^{K O}$ livers (data not shown). Thus, the extreme alterations to Rps19bp 1 and Cacna1i expression upon Cre-mediated deletion of exons 2 and 3 of Atf 4 are likely a secondary result of disruptions to the chromosome 15 architecture in this region (discussed further below).

\section{Discussion}

Recent studies demonstrate that ATF4 is a downstream target of mTORC1 signaling in proliferating cells [24-26]. In non-proliferative settings such as the liver, ATF4 function has been 
largely studied in the context of stress, including amino acid limitation, ER stress, and in models of obesity and fatty liver [28, 51, 57-59]. Here, we define ATF4 as a novel metabolic effector of physiological mTORC1 activation with feeding in the liver and insulin in primary hepatocytes. While the mTORC1-ATF4 axis has recently been found to be activated in the pancreatic islets of mice with $\beta$ cell-specific genetic ablation of the secretory peptidase Furin [60], our findings place ATF4 as a downstream target of mTORC1 in a non-proliferative metabolic tissue activated in response to hormonal cues. Dynamic functional regulation of hepatic mTOR signaling with fasting and feeding was reported in neonatal pigs two decades ago, with protein synthesis as the primary metabolic output [8]. However, to date, only SREBP1c has been implicated as a downstream transcriptional effector of $\mathrm{mTORC} 1$ signaling in the liver activated in response to feeding and insulin $[4,6,20]$, which pales in comparison to the extensive transcriptional network described for mTORC1 signaling in proliferating cells [61]. While more work is necessary to fully define how mTORC1 alters hepatic metabolism in response to feeding, this current study demonstrates that the canonical stress-responsive transcription factor ATF4 can be alternatively activated downstream of $\mathrm{mTORC} 1$ the liver to control specific metabolic processes, including amino acid metabolism.

Given the robust mTORC1-ATF4-mediated regulation of genes of the serine/glycine synthesis and one-carbon metabolism pathways, which provide essential substrates for de novo purine synthesis, observed in the intact liver and isolated hepatocytes in this study, we hypothesized that this signaling axis would control these processes in response to insulin. Compared to other tissues, the liver has a massive protein synthetic capacity, as approximately $85-90 \%$ of the protein content of blood serum originates in this organ [62]. Such a demand for protein synthesis would necessitate a robust program of ribosome biogenesis that includes the synthesis of nucleotides essential for rRNA production. Indeed, we observed that mTORC1 promotes the de novo synthesis of both purine and pyrimidine nucleotides in primary 
hepatocytes, consistent with previous studies in proliferating cells [11, 12, 24]. However, insulin and mTORC1 stimulated these processes in an ATF4-independent manner. A previous study in cell culture models identified other transcription factors, in addition to ATF4, that stimulated de novo purine synthesis in response to mTORC1 activation, which could underlie this regulation in hepatocytes [63]. We provide evidence that both feeding and insulin stimulate hepatic CAD phosphorylation in an mTORC1-dependent manner, an activating modification on this enzyme leading to increased flux through the de novo pyrimidine synthesis pathway $[11,12]$. Further studies are necessary to better elucidate the mechanism(s) of mTORC1-driven de novo nucleotide synthesis in the liver, but the ability of insulin to stimulate this process occurs independent of ATF4, at least in primary hepatocytes. Lastly, it is worth noting that the fractional enrichment of de novo synthesized nucleotides in total hepatocyte RNA measured in our study was much lower than expected. Thus, factors influencing ribosome synthesis and turnover in the liver, along with specific effects on other RNA species such as tRNAs, which are also critical to support protein synthesis, are important areas for future investigation.

ATF4 is known to induce the expression of genes encoding nearly every enzyme of nonessential amino acid synthesis, a function documented downstream of both the ISR and mTORC1 activation in other settings [22, 24-26, 42]. Despite evident regulation of the serine synthesis genes PHGDH, PSAT1, and PSPH by insulin signaling through mTORC1 and ATF4 in primary hepatocytes, we failed to detect any regulated flux through the serine synthesis pathway, which was extremely low under all conditions tested. Our findings are consistent with data from a recent study that showed the fractional enrichment of serine $(m+3)$ synthesized from ${ }^{13} \mathrm{C}_{6}$-glucose in the liver was minimal $(<1 \%)$ [64]. Thus, despite clear regulation of the serine synthesis enzymes, hepatocytes might rely predominantly on exogenous serine. In contrast to serine synthesis, we did observe insulin and ATF4-dependent synthesis of aspartate and alanine, but the genes encoding the aminotransferase enzymes for aspartate and alanine 
synthesis, which have been established as transcriptional targets of ATF4 in other settings [25, $26,42,49]$ were not regulated by insulin or ATF4 in hepatocytes. These data highlight the importance of measuring metabolic flux, as changes in enzyme expression do not always reflect changes in metabolic activity, which is also strongly influenced by allosteric regulation and concentrations of metabolic substrates, cofactors, and products.-Future studies are warranted to determine the mechanism by which ATF4 influences aspartate and alanine synthesis in response to insulin and $\mathrm{mTORC} 1$ signaling in primary hepatocytes.

Unbiased metabolomics and subsequent metabolic flux analyses demonstrated that the hepatocyte insulin-mTORC1-ATF4 pathway stimulates SAM production within the methionine cycle, as well as the transsulfuration pathway, which shunts off of the methionine cycle. While a recent study demonstrated that $\mathrm{mTORC} 1$ regulates SAM synthesis in proliferating cells through c-Myc-dependent control of Mat2a expression [48], the levels of transcripts encoding the MAT enzymes were unaffected by insulin signaling in hepatocytes. Hepatic MAT activity can also be attenuated by reactive oxygen species [46], which are elevated in other models of ATF4 deficiency [22]. However, methionine tracing into SAM, as a more direct assay of MAT activity, indicated that this enzymatic step is unlikely to be the major point of regulation by insulin-ATF4 signaling in hepatocytes. Importantly, the methionine cycle is calibrated to the THF cycle via SAM allosterically inhibiting MTHFR, which serves as the entry point of serine-derived one carbon units into the cycle [47]. It is possible that the defect in insulin-stimulated SAM synthesis observed with ATF4 knockdown in hepatocytes stems from a perturbation in redox that affects MTHFR activity or from other defects in the THF cycle. We also observed a striking insulinstimulated increase in the synthesis of the transsulfuration pathway intermediate cystathionine downstream of mTORC1 and ATF4. Within the transsulfuration pathway, insulin, mTORC1, and ATF4 were found to regulate expression of the second enzyme in the pathway, cystathionine $\gamma$ lyase, encoded by $C t h$, but not the first enzyme off of the methionine cycle, cystathionine $\beta$ - 
synthase, encoded by Cbs, consistent with studies on ATF4 activated as part of the ISR [65]. Interestingly, CBS activity can be allosterically activated by SAM [66], raising the possibility that the SAM and cystathionine changes might be mechanistically linked. However, insulin signaling leading to ATF4 activation did not influence methionine tracing into SAM but did stimulate methionine tracing into cystathionine, indicating that the effects of ATF4 on these two metabolites are separable in hepatocytes. Given that the insulin-mTORC1-ATF4 pathway induces Cth expression in hepatocytes, it seems likely that the observed changes in steady state levels and metabolic flux into cystathionine are mediated through this regulation. This is also consistent with the fact that $\alpha$-ketobutyrate produced in the $\mathrm{CTH}$ reaction is similarly regulated. It should be noted that technical issues prevented us from reliably measuring the synthesis of cysteine, the other product of this reaction. As Cth was the only gene in these interconnected metabolic pathways found to be induced with insulin in an ATF4-dependent manner, it is possible that regulation of the transsulfuration pathway at the CTH step influences both the THF and methionine cycles to account for increased methionine disposal through this route. This regulation of the transsulfuration pathway has potentially important metabolic consequences in the liver, as its product cysteine is required for the synthesis of protein, glutathione, and taurine, the latter of which contributes to hepatic bile acid production [66, 67].

While we were able to identify and validate feeding-induced ATF4 target genes in the liver using the $L A t f 4^{K O}$ model, a limitation of our study is the dramatic alterations to Rps19bp1 and Cacna1i expression found to accompany Cre-mediated deletion of Atf4 in this model. These changes make it more difficult to definitively assign specific phenotypes to loss of ATF4 function in the liver of these mice. For example, while a p53-mediated stress response has been observed in other settings of ATF4 loss [68-70], it is possible that the robust upregulation of p53 and its gene targets observed in the $L A t f 4^{K O}$ livers and cultured hepatocytes could stem from loss of Rps 19bp1 expression. The protein encoded by this gene, also known as active regulator of 
SIRT1 (AROS), acts with SIRT1 to deacetylate and inactivate p53 [71]. However, transient knockdown of Rps19bp1/Aros was not sufficient to induce the expression of p53 target genes in primary hepatocytes in our study. Importantly, similar alterations to the expression of Rps $19 b p 1$ and Cacna1i have recently been observed in RNA-Seq studies of skeletal muscle from musclespecific Atf4 knockout mice ( $m A t f 4^{K O}$ ) (C.M. Adams, personal communication). It is worth noting that $m A t f 4^{K O}$ muscle does not exhibit increased expression of p53 or its gene targets $[72,73]$, indicating that p53 activation does not coincide with reduced Rps19bp1 expression in all settings. The apparent increase in Cacna1i transcripts in $m A t f 4^{K O}$ muscle has been characterized to result from a non-natural fusion transcript consisting of Atf4 exon 1 fused to Cacna1i exon 2, which produces an out-of-frame fusion transcript, without alterations to the normal full-length Cacna1i mRNA. Thus, it is unlikely that the effects on this transcript yield functional consequences. Nonetheless, future studies employing these and other mouse models that genetically ablate Atf4 should consider the potential for similar effects on neighboring genes.

In summary, we describe ATF4 as a novel metabolic effector of hepatic mTORC1 signaling in response to insulin. While there are likely other downstream mediators of the mTORC1dependent feeding response yet to be defined, this study advances our understanding of how mTORC1 exerts metabolic control in a physiological setting. Notably, hepatic mTORC1 and ATF4 are both upregulated in obesity [74-76]. It will be interesting in future studies to determine the relative contribution of stress signaling and mTORC1 signaling to ATF4 activation in this setting, perhaps revealing how adaptive regulation of ATF4 with feeding becomes maladaptive when chronically engaged in disease.

\section{Acknowledgements}


We thank Tracy G. Anthony for advice on ATF4 antibodies and mouse models and Tiffany Horng, Sudha Biddinger, Nada Kalaany, Gyan Prakash, Matthew Miller, and members of the Manning lab for advice and technical assistance. This research was supported by grants from the NIH: Joslin Diabetes Center T32-NK007260 (V.B.), R35-CA197459 (B.D.M.), P01CA120964 (B.D.M. and J.A.), and R01-AR071762 and R01-AG060637 (C.M.A.); the Congressionally Directed Medical Research Program on Tuberous Sclerosis Complex award no. W81XWH-18-1-0659 (B.D.M.); U.S. Department of Veteran Affairs grant I01BX00976 (C.M.A.); and a research grant from Zafgen (B.D.M.). These funders were not involved in the design, execution, or interpretation of this study. C.M.A. is a shareholder, director, and officer of Emmyon, Inc. B.D.M. is a shareholder and scientific advisory board member of Navitor Pharmaceuticals. All other authors declare no competing financial interests.

\section{Author Contributions}

V.B. conceived of the project, performed experiments and data analysis and wrote the manuscript. Y.C., K.K., G.H. and J.H.H performed experiments. V.B-B. and S.H.S. analyzed the RNA-sequencing. J.M.A. and I.B.S. performed mass spectrometry. C.M.A. supplied the Atff ${ }^{f / / f l}$ mice and critical insights into ATF4. B.D.M. conceived of and supervised the project and wrote the manuscript. 


\section{References}

[1] R. L. Smith, M. R. Soeters, R. C. I. Wüst, and R. H. Houtkooper, "Metabolic Flexibility as an Adaptation to Energy Resources and Requirements in Health and Disease," Endocrine Reviews, vol. 39, no. 4, pp. 489-517, 2018/04/24/ 2018, doi: 10.1210/er.201700211.

[2] J. J. Howell and B. D. Manning, "mTOR couples cellular nutrient sensing to organismal metabolic homeostasis," (in eng), Trends Endocrinol Metab, vol. 22, no. 3, pp. 94-102, Mar 2011, doi: 10.1016/j.tem.2010.12.003.

[3] R. A. Saxton and D. M. Sabatini, "mTOR Signaling in Growth, Metabolism, and Disease," (in en), Cell, vol. 168, no. 6, pp. 960-976, 2017/03// 2017, doi: 10.1016/j.cell.2017.02.004.

[4] Jessica L. Yecies et al., "Akt Stimulates Hepatic SREBP1c and Lipogenesis through Parallel mTORC1-Dependent and Independent Pathways," Cell Metabolism, vol. 14, no. 1, pp. 21-32, 2011/07/06/ 2011, doi: 10.1016/j.cmet.2011.06.002.

[5] S. Sengupta, T. R. Peterson, M. Laplante, S. Oh, and D. M. Sabatini, "mTORC1 controls fasting-induced ketogenesis and its modulation by ageing," (in eng), Nature, vol. 468, no. 7327, pp. 1100-4, Dec 2010, doi: 10.1038/nature09584.

[6] T. R. Peterson et al., "mTOR complex 1 regulates lipin 1 localization to control the SREBP pathway," (in eng), Cell, vol. 146, no. 3, pp. 408-20, Aug 2011, doi: 10.1016/j.cell.2011.06.034.

[7] T. Naito, A. Kuma, and N. Mizushima, "Differential contribution of insulin and amino acids to the mTORC1-autophagy pathway in the liver and muscle," (in eng), J Biol Chem, vol. 288, no. 29, pp. 21074-81, Jul 2013, doi: 10.1074/jbc.M113.456228.

[8] S. R. Kimball, L. S. Jefferson, H. V. Nguyen, A. Suryawan, J. A. Bush, and T. A. Davis, "Feeding stimulates protein synthesis in muscle and liver of neonatal pigs through an mTOR-dependent process," (in eng), Am J Physiol Endocrinol Metab, vol. 279, no. 5, pp. E1080-7, Nov 2000, doi: 10.1152/ajpendo.2000.279.5.E1080.

[9] X. M. Ma and J. Blenis, "Molecular mechanisms of mTOR-mediated translational control," (in eng), Nat Rev Mol Cell Biol, vol. 10, no. 5, pp. 307-18, May 2009, doi: 10.1038/nrm2672.

[10] A. J. Valvezan and B. D. Manning, "Molecular logic of mTORC1 signalling as a metabolic rheostat," (in En), Nature Metabolism, vol. 1, no. 3, p. 321, 2019/03// 2019, doi: 10.1038/s42255-019-0038-7. 
[11] I. Ben-Sahra, J. J. Howell, J. M. Asara, and B. D. Manning, "Stimulation of de Novo Pyrimidine Synthesis by Growth Signaling Through mTOR and S6K1," (in en), Science, vol. 339, no. 6125, pp. 1323-1328, 2013/03/15/ 2013, doi: 10.1126/science.1228792.

[12] A. M. Robitaille et al., "Quantitative Phosphoproteomics Reveal mTORC1 Activates de Novo Pyrimidine Synthesis," (in en), Science, vol. 339, no. 6125, pp. 1320-1323, 2013/03/15/ 2013, doi: 10.1126/science.1228771.

[13] J. B. Brugarolas, F. Vazquez, A. Reddy, W. R. Sellers, and W. G. Kaelin, "TSC2 regulates VEGF through mTOR-dependent and -independent pathways," (in eng), Cancer Cell, vol. 4, no. 2, pp. 147-58, Aug 2003.

[14] C. C. Hudson et al., "Regulation of hypoxia-inducible factor 1alpha expression and function by the mammalian target of rapamycin," (in eng), Mol Cell Biol, vol. 22, no. 20, pp. 7004-14, Oct 2002, doi: 10.1128/mcb.22.20.7004-7014.2002.

[15] K. Düvel et al., "Activation of a Metabolic Gene Regulatory Network Downstream of mTOR Complex 1," Molecular Cell, 10.1016/j.molcel.2010.06.022 vol. 39, no. 2, pp. 171 183, 2010, doi: papers3://publication/doi/10.1016/j.molcel.2010.06.022.

[16] L. He et al., "mTORC1 Promotes Metabolic Reprogramming by the Suppression of GSK3-Dependent Foxk1 Phosphorylation," (in eng), Mol Cell, vol. 70, no. 5, pp. 949960.e4, 06 2018, doi: 10.1016/j.molcel.2018.04.024.

[17] T. Porstmann et al., "SREBP activity is regulated by mTORC1 and contributes to Aktdependent cell growth," (in eng), Cell Metab, vol. 8, no. 3, pp. 224-36, Sep 2008, doi: 10.1016/j.cmet.2008.07.007.

[18] S. J. Ricoult, J. L. Yecies, I. Ben-Sahra, and B. D. Manning, "Oncogenic PI3K and K-Ras stimulate de novo lipid synthesis through mTORC1 and SREBP," (in eng), Oncogene, vol. 35 , no. 10 , pp. 1250-60, Mar 2016, doi: 10.1038/onc.2015.179.

[19] Y. Zhang et al., "Coordinated regulation of protein synthesis and degradation by mTORC1," (in eng), Nature, vol. 513, no. 7518, pp. 440-3, Sep 2014, doi: 10.1038/nature13492.

[20] S. Li, M. S. Brown, and J. L. Goldstein, "Bifurcation of insulin signaling pathway in rat liver: mTORC1 required for stimulation of lipogenesis, but not inhibition of gluconeogenesis," (in eng), Proc Natl Acad Sci U S A, vol. 107, no. 8, pp. 3441-6, Feb 2010, doi: 10.1073/pnas.0914798107.

[21] K. Pakos-Zebrucka, I. Koryga, K. Mnich, M. Ljujic, A. Samali, and A. M. Gorman, "The integrated stress response," (in eng), EMBO Rep, vol. 17, no. 10, pp. 1374-1395, Oct 2016, doi: 10.15252/embr.201642195. 
[22] H. P. Harding et al., "An integrated stress response regulates amino acid metabolism and resistance to oxidative stress," (in eng), Mol Cell, vol. 11, no. 3, pp. 619-33, Mar 2003.

[23] C. M. Adams, "Role of the transcription factor ATF4 in the anabolic actions of insulin and the anti-anabolic actions of glucocorticoids," (in eng), J Biol Chem, vol. 282, no. 23, pp. 16744-53, Jun 2007, doi: 10.1074/jbc.M610510200.

[24] I. Ben-Sahra, G. Hoxhaj, S. J. Ricoult, J. M. Asara, and B. D. Manning, "mTORC1 induces purine synthesis through control of the mitochondrial tetrahydrofolate cycle," (in eng), Science, vol. 351, no. 6274, pp. 728-33, Feb 2016, doi: 10.1126/science.aad0489.

[25] Y. Park, A. Reyna-Neyra, L. Philippe, and C. C. Thoreen, "mTORC1 Balances Cellular Amino Acid Supply with Demand for Protein Synthesis through Post-transcriptional Control of ATF4," (in en), Cell Reports, vol. 19, no. 6, pp. 1083-1090, 2017/05// 2017, doi: 10.1016/j.celrep.2017.04.042.

[26] M. E. Torrence et al., "The mTORC1-mediated activation of ATF4 promotes protein and glutathione synthesis downstream of growth signals," (in eng), Elife, vol. 10, Mar 2021, doi: $10.7554 /$ eLife.63326.

[27] S. M. Ebert et al., "Stress-induced skeletal muscle Gadd45a expression reprograms myonuclei and causes muscle atrophy," (in eng), J Biol Chem, vol. 287, no. 33, pp. 27290-301, Aug 2012, doi: 10.1074/jbc.M112.374777.

[28] M. E. Fusakio et al., "Transcription factor ATF4 directs basal and stress-induced gene expression in the unfolded protein response and cholesterol metabolism in the liver," (in eng), Mol Biol Cell, vol. 27, no. 9, pp. 1536-51, May 2016, doi: 10.1091/mbc.E16-010039.

[29] Y. Wang et al., "Hepatic ANGPTL3 regulates adipose tissue energy homeostasis," (in eng), Proc Natl Acad Sci U S A, vol. 112, no. 37, pp. 11630-5, Sep 2015, doi: 10.1073/pnas.1515374112.

[30] M. Yuan, S. B. Breitkopf, X. Yang, and J. M. Asara, "A positive/negative ion-switching, targeted mass spectrometry-based metabolomics platform for bodily fluids, cells, and fresh and fixed tissue," (in eng), Nat Protoc, vol. 7, no. 5, pp. 872-81, Apr 2012, doi: 10.1038/nprot.2012.024.

[31] M. Martin, "Cutadapt removes adapter sequencing from high-throughput sequencing reads.," EMBnet.journal, 2011.

[32] A. Dobin et al., "STAR: ultrafast universal RNA-seq aligner," (in eng), Bioinformatics, vol. 29, no. 1, pp. 15-21, Jan 2013, doi: 10.1093/bioinformatics/bts635. 
[33] M. I. Love, W. Huber, and S. Anders, "Moderated estimation of fold change and dispersion for RNA-seq data with DESeq2," (in eng), Genome Biol, vol. 15, no. 12, p. 550, 2014, doi: 10.1186/s13059-014-0550-8.

[34] G. Yu, L. G. Wang, Y. Han, and Q. Y. He, "clusterProfiler: an R package for comparing biological themes among gene clusters," (in eng), OMICS, vol. 16, no. 5, pp. 284-7, May 2012, doi: 10.1089/omi.2011.0118.

[35] C. C. Thoreen, L. Chantranupong, H. R. Keys, T. Wang, N. S. Gray, and D. M. Sabatini, "A unifying model for mTORC1-mediated regulation of mRNA translation," (in eng), Nature, vol. 485, no. 7396, pp. 109-13, May 2012, doi: 10.1038/nature11083.

[36] B. Selvarajah et al., "mTORC1 amplifies the ATF4-dependent de novo serine-glycine pathway to supply glycine during TGF- $\beta$," (in eng), Sci Signal, vol. 12, no. 582, May 2019, doi: 10.1126/scisignal.aav3048.

[37] J. Ye et al., "GCN2 sustains mTORC1 suppression upon amino acid deprivation by inducing Sestrin2," (in eng), Genes Dev, vol. 29, no. 22, pp. 2331-6, Nov 2015, doi: 10.1101/gad.269324.115.

[38] K. J. Condon et al., "Genome-wide CRISPR screens reveal multitiered mechanisms through which mTORC1 senses mitochondrial dysfunction," (in eng), Proc Natl Acad Sci U S A, vol. 118, no. 4, Jan 2021, doi: 10.1073/pnas.2022120118.

[39] D. Xu et al., "ATF4-Mediated Upregulation of REDD1 and Sestrin2 Suppresses mTORC1 Activity during Prolonged Leucine Deprivation," (in eng), J Nutr, vol. 150, no. 5, pp. 1022-1030, 05 2020, doi: 10.1093/jn/nxz309.

[40] L. Luo et al., "De-silencing Grb10 contributes to acute ER stress-induced steatosis in mouse liver," (in eng), J Mol Endocrinol, vol. 60, no. 4, pp. 285-297, 05 2018, doi: 10.1530/JME-18-0018.

[41] J. Pelletier, G. Thomas, and S. Volarević, "Ribosome biogenesis in cancer: new players and therapeutic avenues," (in eng), Nat Rev Cancer, vol. 18, no. 1, pp. 51-63, Jan 2018, doi: $10.1038 /$ nrc.2017.104.

[42] J. Han et al., "ER-stress-induced transcriptional regulation increases protein synthesis leading to cell death," Nature Cell Biology, 10.1038/ncb2738 vol. 15, no. 5, pp. 481-490, 2013, doi: papers3://publication/doi/10.1038/ncb2738.

[43] J. Ye et al., "Pyruvate kinase M2 promotes de novo serine synthesis to sustain mTORC1 activity and cell proliferation," (in eng), Proc Natl Acad Sci U S A, vol. 109, no. 18, pp. 6904-9, May 2012, doi: 10.1073/pnas.1204176109. 
[44] B. Chaneton et al., "Serine is a natural ligand and allosteric activator of pyruvate kinase M2," (in eng), Nature, vol. 491, no. 7424, pp. 458-462, Nov 2012, doi:

10.1038/nature11540.

[45] O. D. Maddocks et al., "Serine starvation induces stress and p53-dependent metabolic remodelling in cancer cells," (in eng), Nature, vol. 493, no. 7433, pp. 542-6, Jan 2013, doi: 10.1038/nature11743.

[46] F. J. Corrales et al., "Regulation of mammalian liver methionine adenosyltransferase," (in eng), J Nutr, vol. 132, no. 8 Suppl, pp. 2377S-2381S, 08 2002, doi: 10.1093/jn/132.8.2377S.

[47] S. M. Sanderson, X. Gao, Z. Dai, and J. W. Locasale, "Methionine metabolism in health and cancer: a nexus of diet and precision medicine," (in eng), Nat Rev Cancer, vol. 19, no. 11 , pp. 625-637, 11 2019, doi: 10.1038/s41568-019-0187-8.

[48] E. Villa et al., "mTORC1 stimulates cell growth through SAM synthesis and m," (in eng), Mol Cell, Mar 2021, doi: 10.1016/j.molcel.2021.03.009.

[49] X. Yang et al., "ATF4 Regulates CD4 + T Cell Immune Responses through Metabolic Reprogramming," (in en), Cell Reports, vol. 23, no. 6, pp. 1754-1766, 2018/05// 2018, doi: 10.1016/j.celrep.2018.04.032.

[50] Y. Park, A. Reyna-Neyra, L. Philippe, and C. C. Thoreen, "mTORC1 Balances Cellular Amino Acid Supply with Demand for Protein Synthesis through Post-transcriptional Control of ATF4," (in eng), Cell Rep, vol. 19, no. 6, pp. 1083-1090, 05 2017, doi: 10.1016/j.celrep.2017.04.042.

[51] I. A. Nikonorova et al., "Obesity challenges the hepatoprotective function of the integrated stress response to asparaginase exposure in mice," (in en), Journal of Biological Chemistry, vol. 292, no. 16, pp. 6786-6798, 2017/04/21/ 2017, doi: 10.1074/jbc.M116.768408.

[52] H. Li et al., "ATF4 deficiency protects mice from high-carbohydrate-diet-induced liver steatosis," (in eng), Biochem J, vol. 438, no. 2, pp. 283-9, Sep 2011, doi: 10.1042/BJ20110263.

[53] G. Xiao et al., "ATF4 Protein Deficiency Protects against High Fructose-induced Hypertriglyceridemia in Mice," (in en), Journal of Biological Chemistry, vol. 288, no. 35, pp. 25350-25361, 2013/08/30/ 2013, doi: 10.1074/jbc.M113.470526.

[54] H. C. Masuoka, "Targeted disruption of the activating transcription factor 4 gene results in severe fetal anemia in mice," (in en), Blood, vol. 99, no. 3, pp. 736-745, 2002/02/01/ 2002, doi: 10.1182/blood.V99.3.736. 
[55] J. R. Knight, A. E. Willis, and J. Milner, "Active regulator of SIRT1 is required for ribosome biogenesis and function," (in eng), Nucleic Acids Res, vol. 41, no. 7, pp. 418597, Apr 2013, doi: 10.1093/nar/gkt129.

[56] Y. Xie, D. Huang, L. Wei, and X. J. Luo, "Further evidence for the genetic association between CACNA1I and schizophrenia.," (in eng), Hereditas, vol. 155, p. 16, 2018, doi: 10.1186/s41065-017-0054-0.

[57] I. A. Nikonorova, E. T. Mirek, C. C. Signore, M. P. Goudie, R. C. Wek, and T. G. Anthony, "Time-resolved analysis of amino acid stress identifies elF2 phosphorylation as necessary to inhibit mTORC1 activity in liver," (in eng), J Biol Chem, vol. 293, no. 14, pp. 5005-5015, 04 2018, doi: 10.1074/jbc.RA117.001625.

[58] R. J. T. Al-Baghdadi et al., "Role of activating transcription factor 4 in the hepatic response to amino acid depletion by asparaginase," (in eng), Sci Rep, vol. 7, no. 1, p. 1272, 04 2017, doi: 10.1038/s41598-017-01041-7.

[59] K. Li et al., "Liver-specific Gene Inactivation of the Transcription Factor ATF4 Alleviates Alcoholic Liver Steatosis in Mice," Journal of Biological Chemistry, 10.1074/jbc.M116.726836 vol. 291, no. 35, pp. 18536-18546, 2016, doi: papers3://publication/doi/10.1074/jbc.M116.726836.

[60] B. Brouwers et al., "Loss of Furin in Beta Cells Induces an mTORC1-ATF4 Anabolic Pathway That Leads to Beta Cell Dysfunction," (in eng), Diabetes, vol. 70, no. 2, pp. 492-503, Feb 2021, doi: 10.2337/db20-0474.

[61] I. Ben-Sahra and B. D. Manning, "mTORC1 signaling and the metabolic control of cell growth," (in en), Current Opinion in Cell Biology, vol. 45, pp. 72-82, 2017/04// 2017, doi: 10.1016/j.ceb.2017.02.012.

[62] E. Trefts, M. Gannon, and D. H. Wasserman, "The liver," (in English), Current Biology, vol. 27, no. 21, pp. R1147-R1151, 2017/11/06/ 2017, doi: 10.1016/j.cub.2017.09.019.

[63] J. T. Cunningham, M. V. Moreno, A. Lodi, S. M. Ronen, and D. Ruggero, "Protein and nucleotide biosynthesis are coupled by a single rate-limiting enzyme, PRPS2, to drive cancer," (in eng), Cell, vol. 157, no. 5, pp. 1088-103, May 2014, doi: 10.1016/j.cell.2014.03.052.

[64] Y. P. Kang et al., "PHGDH supports liver ceramide synthesis and sustains lipid homeostasis," (in eng), Cancer Metab, vol. 8, p. 6, 2020, doi: 10.1186/s40170-02000212-x.

[65] J. G. Dickhout et al., "Integrated stress response modulates cellular redox state via induction of cystathionine $\mathrm{y}$-lyase: cross-talk between integrated stress response and thiol metabolism," (in eng), J Biol Chem, vol. 287, no. 10, pp. 7603-14, Mar 2012, doi: 10.1074/jbc.M111.304576. 
[66] J. I. Sbodio, S. H. Snyder, and B. D. Paul, "Regulators of the transsulfuration pathway," (in eng), Br J Pharmacol, vol. 176, no. 4, pp. 583-593, 02 2019, doi: 10.1111/bph.14446.

[67] H. Ripps and W. Shen, "Review: taurine: a "very essential" amino acid," (in eng), Mol Vis, vol. 18, pp. 2673-86, 2012.

[68] T. Hettmann, K. Barton, and J. M. Leiden, "Microphthalmia due to p53-mediated apoptosis of anterior lens epithelial cells in mice lacking the CREB-2 transcription factor," (in eng), Dev Biol, vol. 222, no. 1, pp. 110-23, Jun 2000, doi: 10.1006/dbio.2000.9699.

[69] M. Horiguchi, S. Koyanagi, A. Okamoto, S. O. Suzuki, N. Matsunaga, and S. Ohdo, "Stress-regulated transcription factor ATF4 promotes neoplastic transformation by suppressing expression of the INK4a/ARF cell senescence factors," (in eng), Cancer Res, vol. 72, no. 2, pp. 395-401, Jan 2012, doi: 10.1158/0008-5472.CAN-11-1891.

[70] M. Horiguchi et al., "Rhythmic control of the ARF-MDM2 pathway by ATF4 underlies circadian accumulation of p53 in malignant cells," (in eng), Cancer Res, vol. 73, no. 8, pp. 2639-49, Apr 2013, doi: 10.1158/0008-5472.CAN-12-2492.

[71] E. J. Kim, J. H. Kho, M. R. Kang, and S. J. Um, "Active regulator of SIRT1 cooperates with SIRT1 and facilitates suppression of p53 activity," (in eng), Mol Cell, vol. 28, no. 2, pp. 277-90, Oct 2007, doi: 10.1016/j.molcel.2007.08.030.

[72] D. K. Fox et al., "p53 and ATF4 mediate distinct and additive pathways to skeletal muscle atrophy during limb immobilization," (in eng), Am J Physiol Endocrinol Metab, vol. 307, no. 3, pp. E245-61, Aug 2014, doi: 10.1152/ajpendo.00010.2014.

[73] S. M. Ebert et al., "Identification and Small Molecule Inhibition of an Activating Transcription Factor 4 (ATF4)-dependent Pathway to Age-related Skeletal Muscle Weakness and Atrophy," (in eng), J Biol Chem, vol. 290, no. 42, pp. 25497-511, Oct 2015, doi: 10.1074/jbc.M115.681445.

[74] L. Khamzina, A. Veilleux, S. Bergeron, and A. Marette, "Increased activation of the mammalian target of rapamycin pathway in liver and skeletal muscle of obese rats: possible involvement in obesity-linked insulin resistance," (in eng), Endocrinology, vol. 146, no. 3, pp. 1473-81, Mar 2005, doi: 10.1210/en.2004-0921.

[75] U. Ozcan et al., "Endoplasmic reticulum stress links obesity, insulin action, and type 2 diabetes," (in eng), Science, vol. 306, no. 5695, pp. 457-61, Oct 2004, doi: 10.1126/science.1103160.

[76] L. Ozcan, J. Cristina de Souza, Alp A. Harari, J. Backs, Eric N. Olson, and I. Tabas, "Activation of Calcium/Calmodulin-Dependent Protein Kinase II in Obesity Mediates Suppression of Hepatic Insulin Signaling," (in en), Cell Metabolism, vol. 18, no. 6, pp. 803-815, 2013/12// 2013, doi: 10.1016/j.cmet.2013.10.011. 


\section{Figure Legends}

Figure 1. Feeding and insulin induce hepatic ATF4 and established gene targets via mTORC1 signaling.

$(A, B)$ Eight-week old male mice were fasted for $12 \mathrm{~h}$ and refed a high carbohydrate diet for $6 \mathrm{~h}$ following pretreatment with vehicle or $10 \mathrm{mg} / \mathrm{kg}$ rapamycin ( $\mathrm{n}=6 / \mathrm{group}$ ). (A) Liver lysates were immunoblotted to assess mTORC1 signaling and ATF4, with mean ATF4 protein to tubulin quantification plotted \pm SEM and normalized to the fasted group (right). (B) Liver gene expression plotted as mean \pm SEM relative to the fasted group. (C) Immunoblot analysis of primary mouse hepatocytes serum starved overnight and treated with 100nM insulin following a 30-minute pretreatment with vehicle (DMSO), 20nM rapamycin, or 750nM Torin1 for the indicated time points. (D) Immunoblot analysis of primary mouse hepatocytes transfected with control (siCT) or a combination of Eif4ebp1 (4ebp1) and Eif4ebp2 (4ebp2) siRNAs, followed by overnight serum starvation and treatment for $8 \mathrm{~h}$ with $100 \mathrm{nM}$ insulin following a 30-minute pretreatment with vehicle, 20nM rapamycin or 500nm Torin1. (E) Immunoblot analysis of primary mouse hepatocytes treated for the indicated time points as in (C). (F) Gene expression in cells treated as in (E) plotted as mean \pm SEM relative to serum-starved cells ( $n=4$ independent experiments). (G) Immunoblot analysis of primary human hepatocytes serum starved overnight and subsequently treated with 100nM insulin following a 30-minute pretreatment with vehicle (DMSO), 20nM rapamycin, or 500nM Torin1 for the indicated time points. $(H)$ Gene expression in cells treated as in $(G)$ plotted as mean \pm SEM $(n=3)$ relative to the serum-starved cells. ${ }^{*} p<0.05,{ }^{* *} p<0.01,{ }^{* * *} p<0.001,{ }^{* * *} p<0.0001$ (one-way ANOVA).

Figure 2. ATF4 is both necessary and sufficient for insulin-mTORC1 signaling to induce select gene targets 
(A) Immunoblot analysis of primary mouse hepatocytes transfected with control (siCT) or Atf4targetting siRNAs followed by overnight serum starvation and treatment with 100nM insulin for 8h. (B) Gene expression in cells treated as in (A) plotted as mean \pm SEM relative to the siCTtransfected serum-starved cells ( $n=3$ independent experiments). (C) Immunoblot analysis of $A t f 4^{f l / f l}$ and $L A t f 4^{K O}$ primary mouse hepatocytes serum starved overnight and subsequently treated with $100 \mathrm{nM}$ insulin for $6 \mathrm{~h}$ following a 30-minute pretreatment with vehicle (DMSO) or 20nM rapamycin. (D) Gene expression in cells treated as in (C) plotted as mean \pm SEM relative

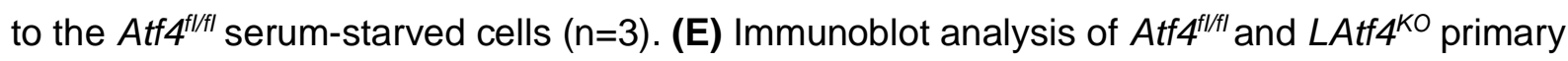
mouse hepatocytes infected with AdGFP or AdATF4 (MOI=10) for 24h. (F) Gene expression from a representative experiment of cells treated as in $(E)$ plotted as mean \pm SEM relative to the Atf4 ${ }^{f / f f l}$ AdGFP sample ( $\left.n=3\right)$. (G) Immunoblot analysis of WT primary mouse hepatocytes infected with AdGFP or AdATF4 (MOI=10) for $24 \mathrm{~h}$ followed by treatment with vehicle (DMSO) or 20nM rapamycin for $8 \mathrm{~h} .{ }^{*} \mathrm{p}<0.05,{ }^{* *} \mathrm{p}<0.01,{ }^{* * *} \mathrm{p}<0.001,{ }^{* * *} \mathrm{p}<0.0001$ (two-way ANOVA (B,D) or one-way ANOVA (F)).

\section{Figure 3. Insulin-mTORC1 signaling induces hepatic de novo nucleotide synthesis in an}

\section{ATF4-independent manner}

(A) Schematic of purine nucleotide highlighting carbon and nitrogen sources for de novo synthesis. (B) Protein normalized peak areas of ${ }^{15} \mathrm{~N}$-glutamine-amide tracing $(2 \mathrm{mM}$, last 30 minutes) into free labeled purine nucleotide pools from primary mouse hepatocytes serum starved overnight and subsequently treated with 100nM insulin following a 30-minute pretreatment with vehicle (DMSO), 20nM rapamycin, or 750nM Torin1 for 8h. Data are plotted as mean $\pm S D$ and are representative of two independent experiments performed in quadruplicate. (C) Fractional enrichment of ${ }^{15} \mathrm{~N}$-glutamine-amide tracing into RNA-derived purine nucleotide isotopologues in primary mouse hepatocytes serum starved overnight and 
subsequently treated with $10 \mathrm{nM}$ insulin following a 30 minute pretreatment with vehicle (DMSO), 20nM rapamycin, or 500nM Torin1 for $8 \mathrm{~h}$ in medium containing $2 \mathrm{mM}{ }^{15} \mathrm{~N}$-glutamine-amide tracer. Data are plotted as mean \pm SD and are representative of two independent experiments performed in triplicate. (D) Protein normalized peak areas of ${ }^{15} \mathrm{~N}$-glutamine-amide tracing (2mM, last 30 minutes) into free labeled purine nucleotide pools from primary mouse hepatocytes transfected with control (siCT) or Atf4-targetting siRNAs 4h after plating, followed by overnight serum starvation and overnight treatment with $10 \mathrm{nM}$ insulin. Data are plotted as mean \pm SD and are representative of two independent experiments performed in quadruplicate. (E) Fractional enrichment of ${ }^{15} \mathrm{~N}$-glutamine-amide tracing into RNA-derived purine nucleotide isotopologues in cells transfected as in (D), followed by overnight serum starvation and treatment with $10 \mathrm{nM}$ insulin in medium containing $2 \mathrm{mM}^{15} \mathrm{~N}$-glutamine-amide tracer for $6 \mathrm{~h}$. Data are plotted as mean $\pm \mathrm{SD}$ and is representative of three independent experiments performed in triplicate. (F) Schematic of pyrimidine nucleotide highlighting carbon and nitrogen sources for de novo synthesis. (G) ${ }^{15} \mathrm{~N}$-glutamine-amide tracing (2mM, last 30 minutes) into free labeled pyrimidine nucleotide pools in primary mouse hepatocytes treated and plotted as in (B). (H) Protein normalized peak areas of ${ }^{15} \mathrm{~N}$-glutamine-amide tracing $(2 \mathrm{mM}$, last 30 minutes $)$ into free labeled pyrimidine nucleotide pools from primary mouse hepatocytes transfected with control (siCT) or Atf4-targetting siRNAs $4 \mathrm{~h}$ after plating, followed by overnight serum starvation and overnight treatment with 100nM insulin. Data are plotted as mean \pm SD and are representative of two independent experiments performed in quadruplicate.

\section{Figure 4. Lack of evidence for regulated serine synthesis in hepatocytes}

(A) Schematic of the serine synthesis pathway. (B) Protein normalized peak areas of ${ }^{15} \mathrm{~N}$ glutamine-amine $(2 \mathrm{mM}$, last $1 \mathrm{~h})$ tracing into labeled serine in primary mouse hepatocytes serum starved overnight and subsequently treated with $100 \mathrm{nM}$ insulin following a 30 -minute 
pretreatment with vehicle (DMSO), 20nM rapamycin, or 750nM Torin1 for $8 \mathrm{~h}$, or where indicated, cycloheximide $(50 \mu \mathrm{M})$ was added with tracer for the last $1 \mathrm{~h}$. Data are plotted as mean $\pm \mathrm{SD}$ and are representative of two independent experiments performed in quadruplicate. (C) Fractional enrichment of serine isotopologues from the experiment in (B). (D) Protein normalized peak areas of ${ }^{15} \mathrm{~N}$-glutamine-amine $(2 \mathrm{mM}$, last $1 \mathrm{~h})$ tracing into labeled serine in primary mouse hepatocytes transfected with control (siCT) or Atf4-targetting siRNAs, followed by overnight serum starvation and overnight treatment with 10nM insulin. Data are plotted as mean \pm SD and are representative of two independent experiments performed in quadruplicate. (E) Fractional enrichment of serine isotopologues from the experiment in (D), plotted as mean \pm SD. (F) Protein normalized peak areas of intracellular serine from primary mouse hepatocytes following incubation in serine and/or glycine-free medium plus $2.5 \%$ dialyzed FBS for $24 \mathrm{~h}$. Data are plotted as mean \pm SD and are representative of two independent experiments performed in triplicate. (G) Fractional enrichment of serine isotopologues in primary mouse hepatocytes treated as in $(F)$ and labeled with ${ }^{15} \mathrm{~N}$-glutamine-amine $(2 \mathrm{mM}$, last $1 \mathrm{~h})$. Data are plotted as mean $\pm \mathrm{SD}$ and is representative of two independent experiments performed in triplicate. $(\mathbf{H})$

Fractional enrichment of isotopologues from primary mouse hepatocytes following incubation in serine-rich or serine-free medium $+2.5 \%$ dialyzed FBS for $20 \mathrm{~h}$ with $10 \mathrm{mM}{ }^{13} \mathrm{C}_{6}$-glucose. Data plotted as mean \pm SD and is representative of two independent experiments performed in quadruplicate.

Figure 5. Insulin stimulates metabolic flux into the methionine cycle and transsulfuration pathway through ATF4.

(A) Steady state metabolomic profiling from primary mouse hepatocytes transfected with control (siCT) or Atf4-targetting siRNAs followed by $100 \mathrm{nM}$ insulin stimulation for $6 \mathrm{~h}$ performed in quadruplicate. The heat map displays a rank order of metabolites significantly $(p<0.05)$ induced 
with insulin in the control cells but not those with Atf4 knockdown. (B) Protein normalized peak areas of the top 6 metabolites $(A)$ plotted as mean \pm SD. (C) Schematic of the interconnections between serine and tetrahydrofolate metabolism, the methionine cycle and the transsulfuration pathway. Metabolites found to be significantly induced by insulin in an ATF4-dependent manner in (A) are highlighted in red. (D) Gene expression analysis of primary mouse hepatocytes transfected with control (siCT) or Atf4-targetting siRNAs followed by overnight serum starvation and treatment with $100 \mathrm{nM}$ insulin for $8 \mathrm{~h}$. Data are plotted as mean $\pm \mathrm{SEM}$ relative to siCTtransfected serum starved cells ( $n=3$ independent experiments). (E) Protein normalized peak areas of $3-{ }^{13} \mathrm{C}$-serine $(400 \mu \mathrm{M}$, last $1 \mathrm{~h})$ tracing into labeled SAM and cystathionine in primary mouse hepatocytes serum starved overnight and subsequently treated with 100nM insulin following a 30-minute pretreatment with vehicle (DMSO), 20nM rapamycin, or 500nM Torin1 for 8h. Data are plotted as mean $\pm S D$ and are representative of two independent experiments performed in triplicate. (F) Protein normalized peak areas of $3-{ }^{13} \mathrm{C}$-serine tracing $(400 \mu \mathrm{M}$, last 1h) in primary mouse hepatocytes transfected with control (siCT) or Atf4-targetting siRNAs followed by overnight serum starvation and treatment with $100 \mathrm{nM}$ insulin for $8 \mathrm{~h}$. Data are plotted as mean $\pm S D$ and are representative of two independent experiments performed in triplicate.

(G) Protein normalized peak areas of labeled SAM from ${ }^{13} \mathrm{C}_{5}$-methionine $(200 \mu \mathrm{M}$, last $1 \mathrm{~h})$ tracing of cells treated as in $(\mathrm{F})$. (H) Fractional enrichment of SAM isotopologues from ${ }^{13} \mathrm{C}_{5^{-}}$ methionine tracing as in (G). (I) Protein normalized peak areas of labeled cystathionine from ${ }^{13} \mathrm{C}_{5}$-methionine tracing as in $(\mathrm{G})$. Data are plotted as mean $\pm \mathrm{SD}$ for tracing studies and are representative of two independent experiments performed in triplicate. ${ }^{*} p<0.05,{ }^{* *} p<0.01$, ${ }^{* * *} p<0.001,{ }^{* * * *} p<0.0001$ (two-way ANOVA).

Figure 6. Characterization of feeding induced transcriptional response in LAtf4 ${ }^{K O}$ mice 
$(\mathrm{A}, \mathrm{B})$ Eight-week old $A t f 4^{f / / f l}$ and $L A t f 4^{K O}$ male mice were fasted for $12 \mathrm{~h}$ and refed a high carbohydrate diet for $6 \mathrm{~h}$ ( $n=5 /$ group). Immunoblots $(A)$ and gene expression analysis $(B)$ of $A t f 4^{f / f l}$ and $L A t f 4^{K O}$ livers. Gene expression data are plotted as mean \pm SEM relative to the Atf4 ${ }^{f / f l}$ fasted group ( $n=5$ mice/group). (C) Principal component analysis of normalized transcripts from RNA-seq of $A t f 4^{f / f l}$ and $L A t f 4^{K O}$ livers treated as in (A). (D) Heat map of normalized transcripts from RNA-seq data significantly $\left(p_{a d j}<0.05\right)$ induced with feeding in the Atff ${ }^{f / / f I}$, but not in the $L A t f 4^{K O}$ livers. (E) Gene set enrichment analysis of the differentially expressed transcripts in fed $L A t f 4^{K O}$ versus $A t f 4^{f / / f l}$ livers. (F) Top-10 most upregulated and down-regulated transcripts plotted as $\log _{2}$ fold change in $L A t f 4^{K O}$ versus $A t f 4^{f / f t}$ livers from fed mice. Asterisks indicate top hits. (G) Gene expression analysis of $A t f 4^{f / / f l}$ and $L A t f 4^{K O}$ livers treated and plotted as in $(\mathrm{A})$. (H) Gene expression analysis of primary mouse hepatocytes transfected with control (siCT) or Atf4targetting siRNAs followed by overnight serum starvation and treatment with 100nM insulin for 8h. Data are plotted as mean \pm SEM relative to the $s i C T$-transfected cells $(n=3$ independent experiments). (I) Gene expression analysis of primary mouse hepatocytes from Atf4 $4^{f / f l}$ and $L A t f 4^{K O}$ mice infected with AdGFP or AdATF4 $(\mathrm{MOI}=10)$ for $24 \mathrm{~h}$. Data from a representative experiment are plotted as mean \pm SEM relative to the $A t f 4^{f / f l}$ AdGFP cells $(n=3)$. (J) Schematic of the Atf4 locus and proximal genes on mouse chromosome 15, along with the modified Atf4 fl/fl locus. ${ }^{*} p<0.05,{ }^{* *} p<0.01,{ }^{* * *} p<0.001,{ }^{* * *} p<0.0001$ (two-way ANOVA (B, G, I) or one-way $\operatorname{ANOVA}(\mathrm{H}))$.

\section{Supplementary Figure Legends}

\section{Supplementary Figure 1. Feeding and insulin induce hepatic ATF4 and established gene}

targets via mTORC1 signaling. (A) Eight-week old male mice were fasted for $12 \mathrm{~h}$ and refed a high carbohydrate diet for $12 \mathrm{~h}$ following pretreatment with vehicle or $10 \mathrm{mg} / \mathrm{kg}$ rapamycin ( $n=6 /$ group). Liver lysates were immunoblotted to assess mTORC1 signaling and ATF4 protein 
levels. (B) LICOR quantification of immunoblots from primary mouse hepatocytes serum starved overnight and subsequently treated with $100 \mathrm{nM}$ insulin following a 30 -minute pretreatment with vehicle (DMSO), 20nM rapamycin, or 750nM Torin1 for the indicated time points. Data are plotted as \pm SEM relative to the serum starved samples ( $n=4$ independent experiments). (C,D) Hepa1-6 and AML-12 cells were serum starved overnight and treated with 100nM insulin following a 30-minute pretreatment with vehicle (DMSO), 20nM rapamycin, or 250nM Torin1 for the indicated time points. ${ }^{*} p<0.05,{ }^{* *} p<0.01,{ }^{* *} p<0.001,{ }^{* * *} p<0.0001$. In panel $\mathrm{C}$, ${ }^{*}$ denote comparisons to serum starved samples at each insulin time point and ${ }^{\#} p<0.05,{ }^{\#} p<0.01$,

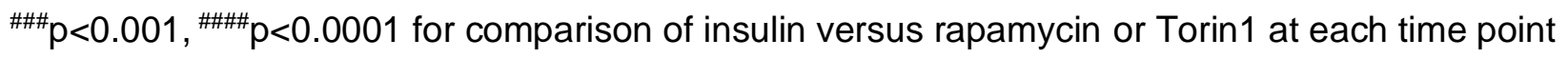
(one-way ANOVA).

\section{Supplementary Figure 2. mTORC1 controls de novo nucleotide synthesis in an ATF4- independent manner}

(A) Protein normalized peak areas of $3-{ }^{13} \mathrm{C}$-serine tracing $(400 \mu \mathrm{M}$, last $1 \mathrm{~h})$ into labeled purine nucleotide pools in primary mouse hepatocytes serum starved overnight and subsequently treated with $100 \mathrm{nM}$ insulin following a 30-minute pretreatment with vehicle (DMSO), 20nM rapamycin, or $500 \mathrm{nM}$ Torin1 for $8 \mathrm{~h}$. Data are plotted as mean \pm SD and are representative of two independent experiments performed in triplicate. (B) Protein normalized peak areas of 3${ }^{13} \mathrm{C}$-serine tracing $(400 \mu \mathrm{M}$, last $1 \mathrm{~h})$ into labeled purine nucleotide pools in primary mouse hepatocytes transfected with control (siCT) siRNAs or those targeting Atf4 or Mthfd2 followed by overnight serum starvation and treatment with $100 \mathrm{nM}$ insulin for $8 \mathrm{~h}$. Data are plotted as mean \pm SD and are representative of two independent experiments performed in triplicate. (C) ${ }^{15} \mathrm{~N}$ glutamine-amide tracing into RNA-derived pyrimidine nucleotide isotopologues in primary mouse hepatocytes serum starved overnight and subsequently treated with 10nM insulin following a 30 minute pretreatment with vehicle (DMSO), 20nM rapamycin, or 500nM Torin1 for 
$8 \mathrm{~h}$ in medium containing $2 \mathrm{mM}^{15} \mathrm{~N}$-glutamine-amide tracer. Fractional enrichment is plotted as mean \pm SD and is representative of two independent experiments performed in triplicate. (D) ${ }^{15} \mathrm{~N}$-glutamine-amide tracing into RNA-derived purine nucleotide isotopologues in primary mouse hepatocytes transfected with control (siCT) or Atf4-targetting siRNAs followed by overnight serum and treatment with $10 \mathrm{nM}$ insulin medium containing $2 \mathrm{mM}^{15} \mathrm{~N}$-glutamine-amide tracer for $8 \mathrm{~h}$. Fractional enrichment is plotted as mean \pm SD and is representative of three independent experiments performed in triplicate.

\section{Supplementary Figure 3. Effects of labeling time on measurements of serine synthesis in primary hepatocytes}

(A-B) Protein normalized peak areas of ${ }^{15} \mathrm{~N}$-glutamine-amine tracing into serine in primary mouse hepatocytes serum starved overnight and subsequently treated with 100nM insulin for 8h. Tracing with $2 \mathrm{mM}^{15} \mathrm{~N}$-glutamine-amine was performed for indicated time points. Peak areas and fractional enrichment of serine isotopologues are plotted as mean $\pm \mathrm{SD}$.

\section{Supplementary Figure 4. Insulin-mTORC1-ATF4 signaling regulates $C$ th and the transsulfuration pathway in primary hepatocytes}

(A) Enrichment analysis of metabolites from Figure 4A, showing the enrichment score for the top 25 KEGG metabolite sets. (B) Steady state levels of glutathione and glutathione disulfide from primary mouse hepatocytes transfected with control (siCT) or Atf4-targetting siRNAs followed by overnight serum starvation and treatment with $100 \mathrm{nM}$ of insulin for $6 \mathrm{~h}$. (C) Gene expression analysis in primary mouse hepatocytes transfected and in (B) followed by overnight serum starvation and treatment with $100 \mathrm{nM}$ insulin for $8 \mathrm{~h}$. Data are plotted as mean \pm SEM relative to siCT serum-starved cells ( $n=3$ independent experiments). (D) Gene expression analysis of primary mouse hepatocytes serum starved overnight and subsequently treated with 
100nM insulin following a 30-minute pretreatment with vehicle (DMSO), 20nM rapamycin, or 750nM Torin1 for the indicated time points. Data are plotted as mean \pm SEM relative to serumstarved cells ( $n=4$ independent experiments). (E) Gene expression analysis of $A t f 4^{f / f l}$ and LAtf4 ${ }^{K O}$ primary mouse hepatocytes serum starved overnight and subsequently treated with $100 \mathrm{nM}$ insulin for $6 \mathrm{~h}$ following a 30-minute pretreatment with vehicle (DMSO) or 20nM rapamycin. Data from a representative experiment are plotted as mean $\pm \operatorname{SEM}(n=3)$ relative to serum-starved $A t f 4^{f l / f l}$ cells. (F) Gene expression analysis of $A t f 4^{f / f l}$ and $L A t f 4^{K O}$ primary mouse hepatocytes infected with AdGFP or AdATF4 (MOI=10) for 24h. Data from a representative experiment are plotted as mean $\pm \operatorname{SEM}(n=3)$ relative to Atff ${ }^{f / / f l}$ AdGFP cells. (G) Protein normalized peak areas of labeled alpha-ketobutyrate $(m+4)$ from the ${ }^{13} \mathrm{C}_{5}$-methionine tracing experiment in Figure 5. Data are plotted as mean \pm SD performed in triplicate. ${ }^{*} p<0.05$, ${ }^{* *} \mathrm{p}<0.01,{ }^{* * *} \mathrm{p}<0.001,{ }^{* * *} \mathrm{p}<0.0001$ (two-way ANOVA $(\mathrm{C}, \mathrm{E})$ or one-way ANOVA $(\mathrm{D}, \mathrm{F})$ ).

\section{Supplementary Figure 5. Insulin and ATF4 induce aspartate and alanine synthesis in}

\section{primary hepatocytes}

(A) Schematic of the glutamic-oxaloacetic transaminase (GOT) reaction to synthesize aspartate.

(B) ${ }^{15} \mathrm{~N}$-glutamine-amine tracing $(2 \mathrm{mM}$, last $1 \mathrm{~h})$ into labeled aspartate $(\mathrm{m}+1)$ in primary mouse hepatocytes serum starved overnight and subsequently treated with 100nM insulin following a 30-minute pretreatment with vehicle (DMSO), 20nM rapamycin, or 750nM Torin1 for 8h, or cycloheximide $(50 \mu \mathrm{M})$ for the last $1 \mathrm{~h}$. Data are plotted as mean $\pm \mathrm{SD}$ and are representative of two independent experiments performed in quadruplicate. (C) Protein normalized peak areas of ${ }^{15} \mathrm{~N}$-glutamine-amine tracing $(2 \mathrm{mM}$, last $1 \mathrm{~h})$ into labeled aspartate $(\mathrm{m}+1)$ in primary mouse hepatocytes transfected with control (siCT) or Atf4-targetting siRNAs followed by overnight serum starvation then treatment with $100 \mathrm{nM}$ insulin. Data are plotted as mean \pm SD and are representative of two independent experiments performed in quadruplicate. (D) Gene 
expression analysis in primary mouse hepatocytes transfected with control (siCT) or Atf4targetting siRNAs followed by overnight serum starvation and treatment with $100 \mathrm{nM}$ insulin for 8h. Data are plotted as mean \pm SEM relative to serum-starved $\operatorname{siCT}$ cells $(\mathrm{n}=3$ independent experiments). (E) Immunoblot analysis of primary mouse hepatocytes treated as in (D). (F) Schematic of the glutamic-pyruvic transamination (GPT) reaction to synthesize alanine. (G) Protein normalized peak areas of ${ }^{15} \mathrm{~N}$-glutamine-amine tracing $(2 \mathrm{mM}$, last $1 \mathrm{~h})$ into labeled alanine $(m+1)$ in primary mouse hepatocytes treated, and with data plotted, as in (B). (H) Protein normalized peak areas of ${ }^{15} \mathrm{~N}$-glutamine-amine tracing into labeled alanine $(\mathrm{m}+1)$ in primary mouse hepatocytes treated, and with data plotted, as in (C). Gene expression analysis in primary mouse hepatocytes treated, and with data plotted, as in (D). ${ }^{*} p<0.05,{ }^{* *} p<0.01$, ${ }^{* * *} p<0.001,{ }^{* * * *} p<0.0001$ (two-way ANOVA).

\section{Supplementary Figure 6. Physiological characterization of LAtf4 ${ }^{K O}$ mice}

(A) Immunoblot analysis of livers from $A t f 4^{f / / f l}$ and $L A t f 4^{K O}$ mice injected with vehicle or $1 \mathrm{mg} / \mathrm{kg}$ tunicamycin for $6 \mathrm{~h}$ to test specificity of the indicated commercially available ATF4 antibodies. (B) Mean \pm SEM body weights of $A t f 4^{f / / f l}$ and $L A t f 4^{K O}$ male mice at 8 weeks ( $\mathrm{n}=15$ mice/group) and 26 weeks of age ( $n=6$ Atf4 $\left.4^{f / f t}, n=7 L A t f 4^{K O}\right)$ are plotted. (C) Glucose and insulin tolerance tests in male $\operatorname{Atf}^{f / / f I}(\mathrm{n}=6)$ and $\operatorname{LAtf} 4^{K O}(\mathrm{n}=7)$ mice at 26 weeks of age. ${ }^{*} \mathrm{p}<0.05$, Students t-test (B).

\section{Supplementary Figure 7. Upregulation of p53 and p53-dependent gene expression in}

\section{LAtf4 $^{K O}$ livers and primary hepatocytes}

(A) Gene set enrichment analysis of the differentially expressed transcripts in fed $L A t f 4^{K O}$ versus Atf $4^{f / f l}$ livers. (B) Immunoblot of livers from eight-week old $A t f 4^{f / f l}$ and $L A t f 4^{K O}$ mice subjected to a $12 \mathrm{~h}$ fast followed by $6 \mathrm{~h}$ refeeding ( $\mathrm{n}=5$ mice/group). (C) Gene expression analysis in livers from 
the mice in $(E)$. Data are plotted as mean \pm SEM relative to the $A t f 4^{f / f f l}$ fasted group $(n=5$

mice/group). (D) Gene expression analysis of primary mouse hepatocytes from Atf $4^{f / f f}$ and $L A t f 4^{K O}$ mice infected with AdGFP or AdATF4 $(\mathrm{MOI}=10)$ for $24 \mathrm{~h}$. Data from a representative experiment are plotted as mean \pm SEM relative to $\operatorname{Atf}^{f / / f l}$ AdGFP cells $(n=3)$. (E) Immunoblot analysis of cells treated as in (G). (F) Gene expression analysis in primary mouse hepatocytes transfected with control (siCT), Atf4, or Rps19bp1-targeting siRNAs for 48h. Data are plotted as mean \pm SEM relative to siCT cells (data are representative of two independent experiments). ${ }^{*} \mathrm{p}<0.05,{ }^{* *} \mathrm{p}<0.01,{ }^{* * *} \mathrm{p}<0.001,{ }^{* * *} \mathrm{p}<0.0001$ (two-way ANOVA (F) or one-way ANOVA (G)).

Supplementary Table 1. List of commercially available ATF4 antibodies tested in in vitro and in vivo.

Supplementary Table 2. Selected reaction monitoring (SRM) table for stable isotope tracing experiments.

Supplementary Table 3. Primer sequences used for qPCR analysis.

Supplementary Table 4. Peak areas and p-values for metabolites in Figure 5A heatmap. Data sorted by fold change of siCT + Insulin/siCT -Insulin. P-value determined by Student's t-test.

Supplementary Table 5. List of genes induced with feeding in the $A t f 4^{f / f t}\left(\log _{2} F C>1, p_{a d j}<0.05\right)$, but not the $L A t f 4^{K O}$ mouse livers. 
Figure 1 Rxiv preprint doi: https://doi.org/10.1101/2021.05.02.442369; this version posted May 3, 2021. The copyright holder for this preprint (which
was not certified by peer review) is the author/funder. All rights reserved. No reuse allowed without permission.

A.

Mouse Livers

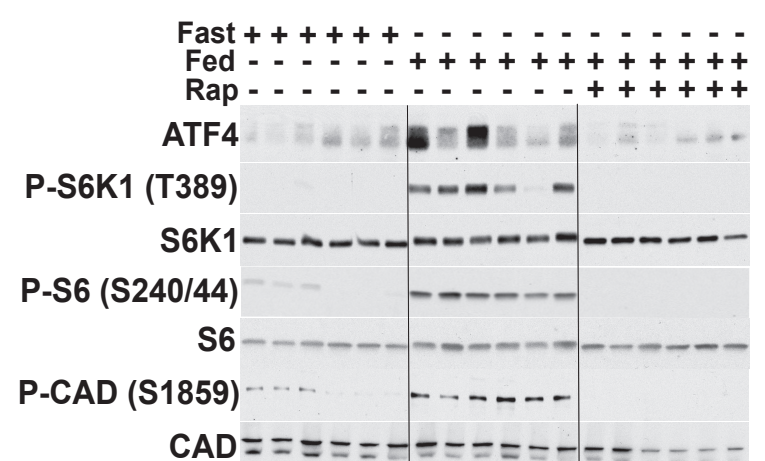

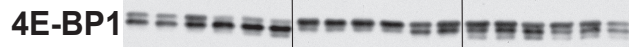

P-elF2 $\alpha$ (S51)

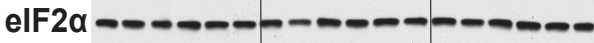

Tubulin

C. Primary Mouse Hepatocytes 0 5' 15'30'1h 2h 5'15'30'1h 2h 5' 15'30'1 $2 \mathrm{~h}$ Insulin +++++++++++++++

Rap - - - - - + + + + - - - -

Torin1 - - - - . - - + + + + ATF4 (light)

ATF4 (dark)

P-S6K1 (T389)

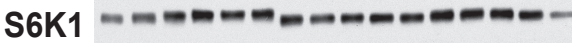

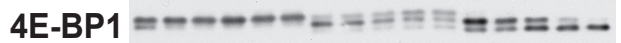

P-AKT (S473)

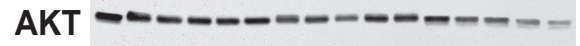

P-elF2 $\alpha(\mathrm{S} 51)$

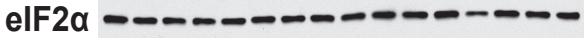

Tubulin - -
B.

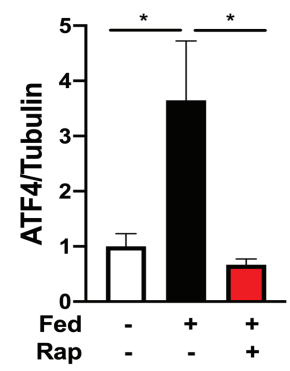

Mthfd2

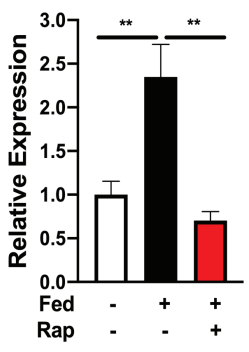

Psph
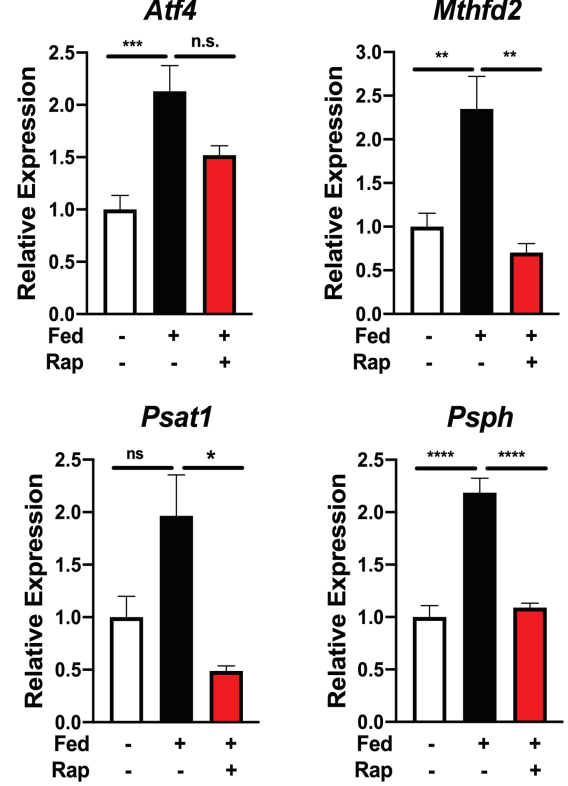

Phgdh

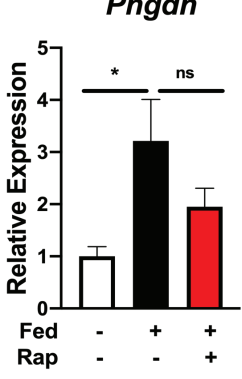

Pepck

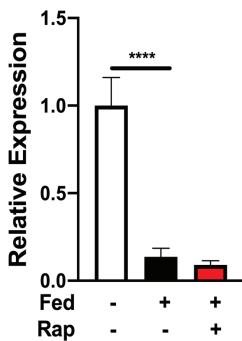

D. Primary Mouse Hepatocytes

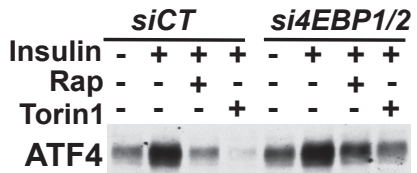

P-S6K1 (T389) - -

S6K1 $=--m-$ 4E-BP1 (light)

4E-BP1 (dark) $=-$

4E-BP2

P-elF2a (S51) - - - -

elF2 $\alpha-0-0-0-$

Tubulin -ோ-ைーー
E.

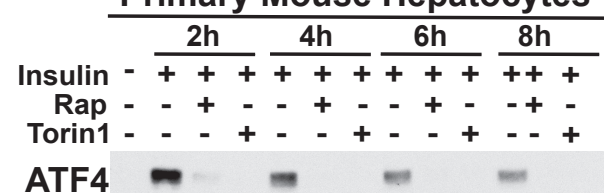

MTHFD2

PHGDH

PSAT1

PSPH

P-AKT (S473) - -

AKT $-----=--\div$

P-S6K1 (T389)

S6K1

P-CAD (S1859)

CADニーニニーのこーこーここニ

4E-BP1 $=-=-=--=-=$

P-elF2 $\alpha$ (S51)

elF2 $\alpha$

Tubulin
F.
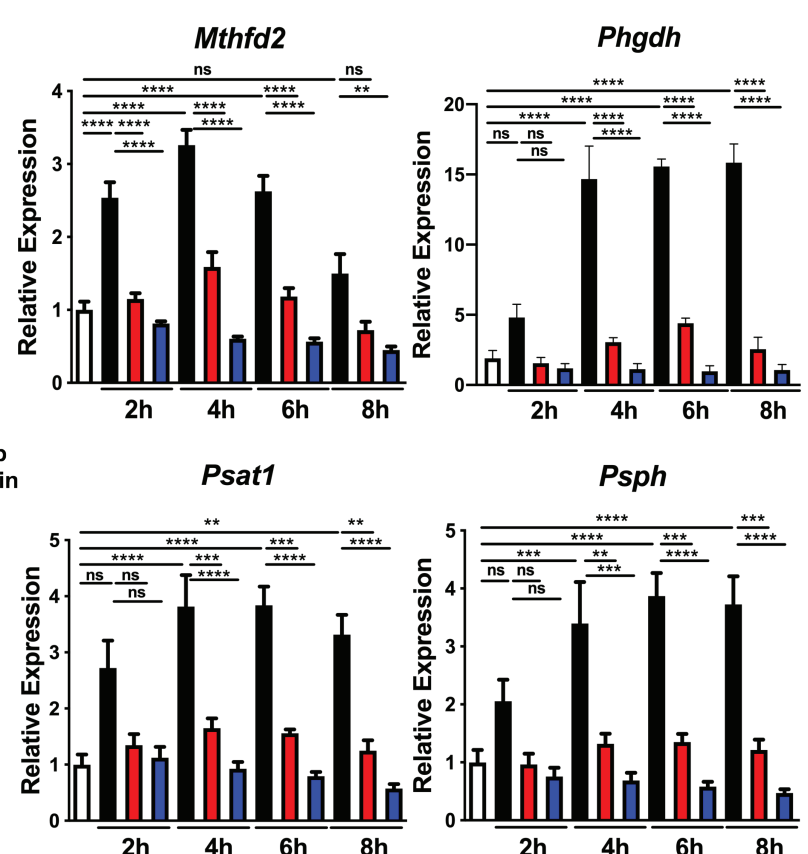

G. Primary Human Hepatocytes $\mathbf{H}$.

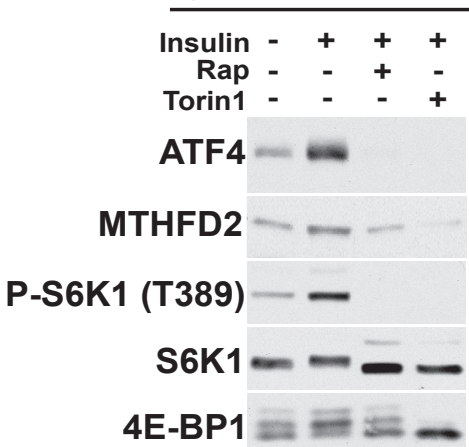

P-elF2a (S51) _

elF2 $\alpha-\infty-$

Tubulin - -
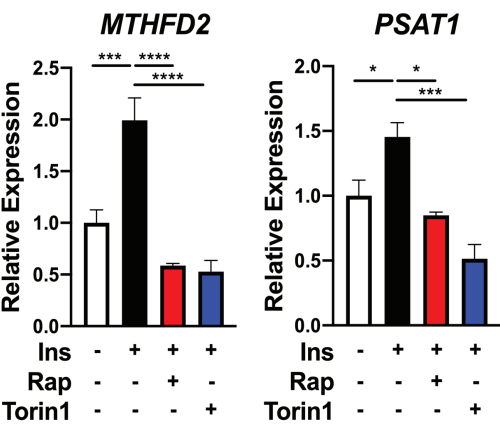

PSPH

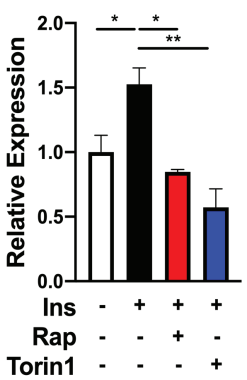



Figure $\begin{array}{r}\text { bioRxiv preprint dol: https://doi.org/10.1101/2021.05.02.442369; this version posted May } 3,2021 . \text { The copyright holder for this preprint (which } \\ \text { was not certified by peer review) is the author/funder. All rights reserved. No reuse allowed without permission. }\end{array}$

$$
\begin{aligned}
& \text { Insulin } \frac{\text { seCT }}{-+} \frac{\text { siAtf4 }}{-+} \\
& \text { ATF4 } \\
& \text { MTHFD2 - - } \\
& \text { PHGDH - - } \\
& \text { PSAT1 - - } \\
& \text { PSPH - - } \\
& \text { P-S6K1 (T389) - - } \\
& \mathrm{S} 6 \mathrm{~K} 1-\infty-= \\
& \text { 4E-BP1 }== \\
& \text { Tubulin - - }
\end{aligned}
$$

c.

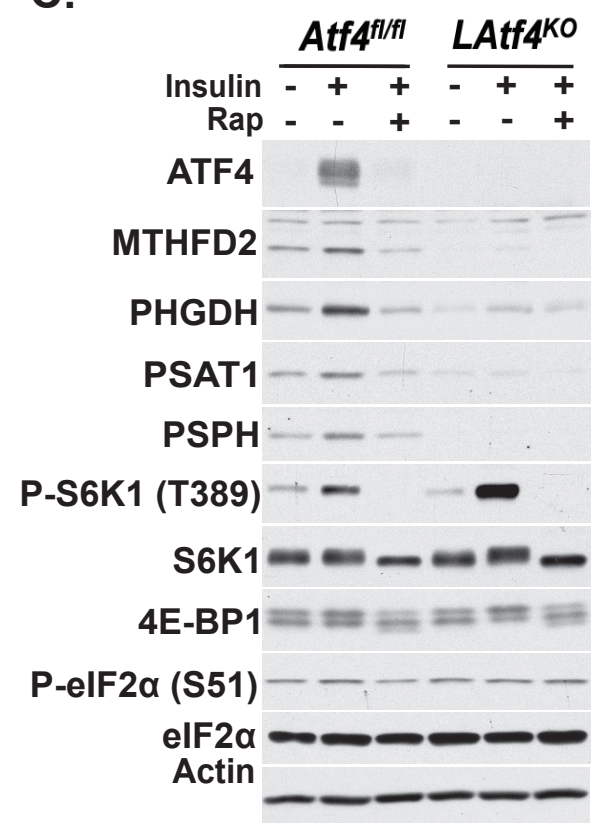

F.

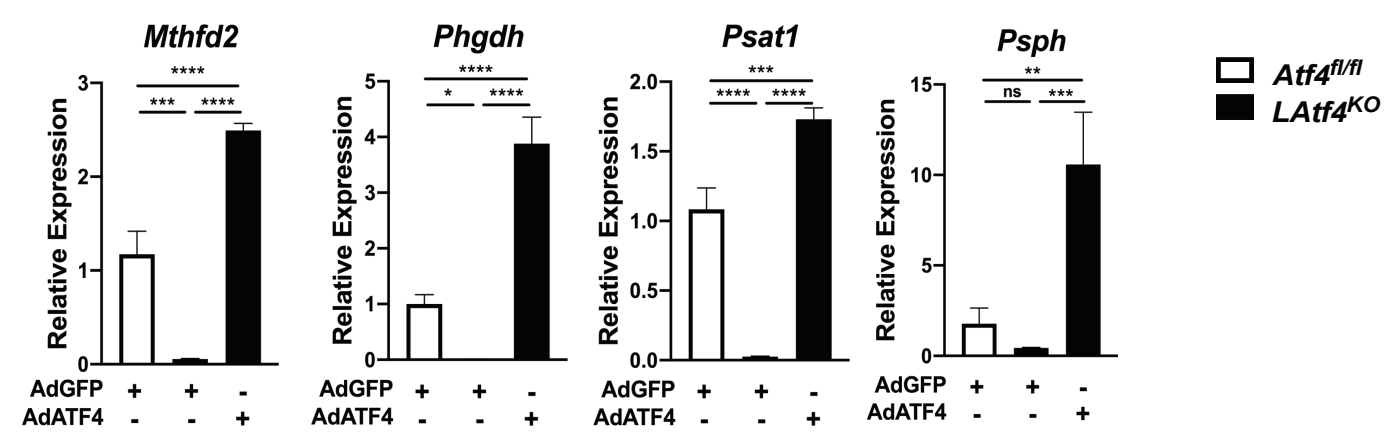

C.
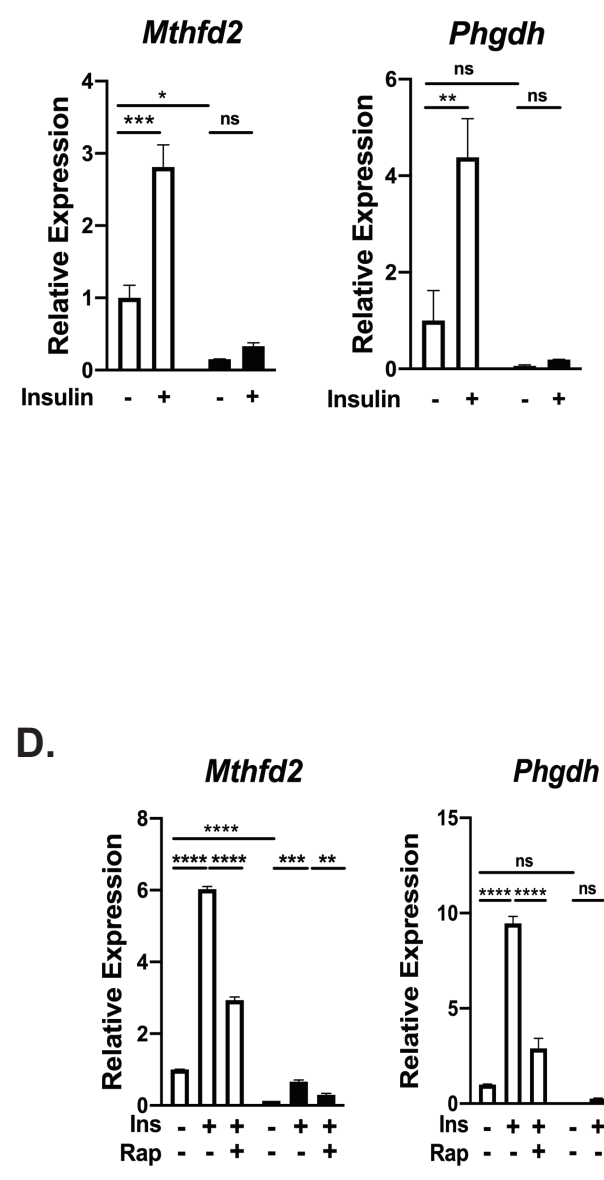
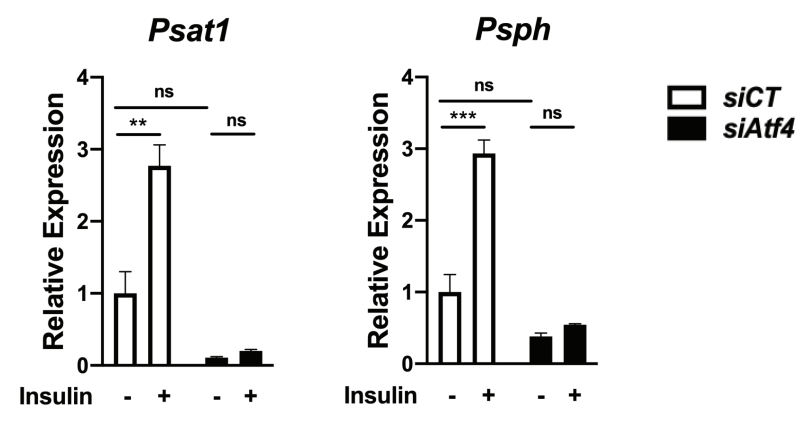

D.
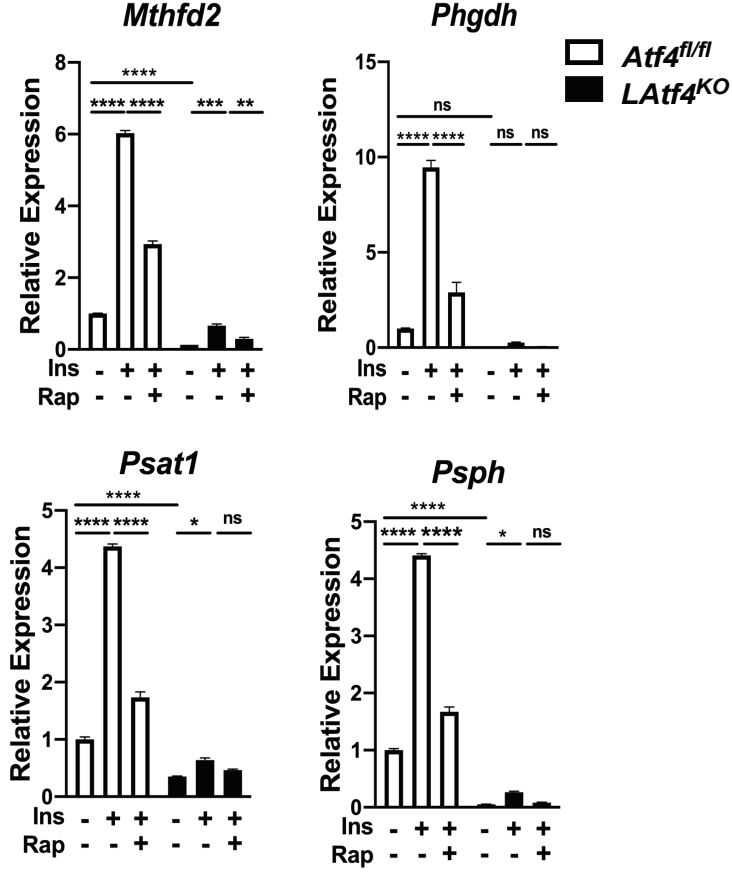

G.

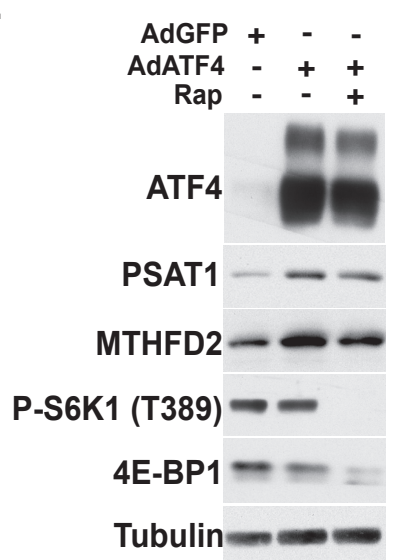

E.

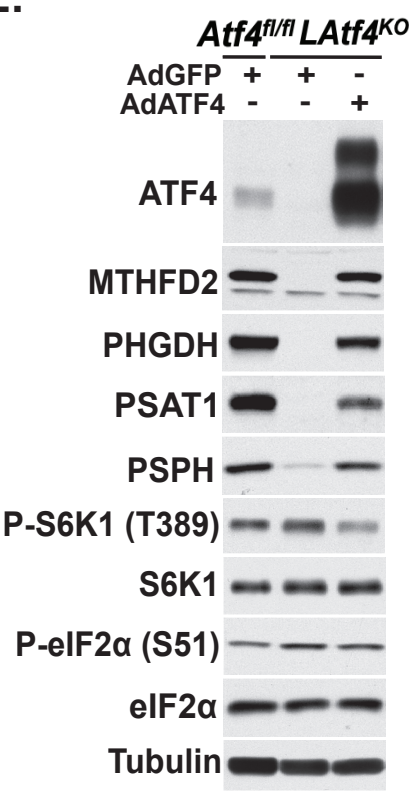



Figure boRxiv preprint doi: https://doi.org/10.1101/2021.05.02.442369; this version posted May 3, 2021. The copyright holder for this preprint (which
was not certified by peer review) is the author/funder. All rights reserved. No reuse allowed without permission.

${ }^{15} \mathrm{~N}$-Glutamine
A.

Purine Nucleotide

Bicarbonate

(Amide)

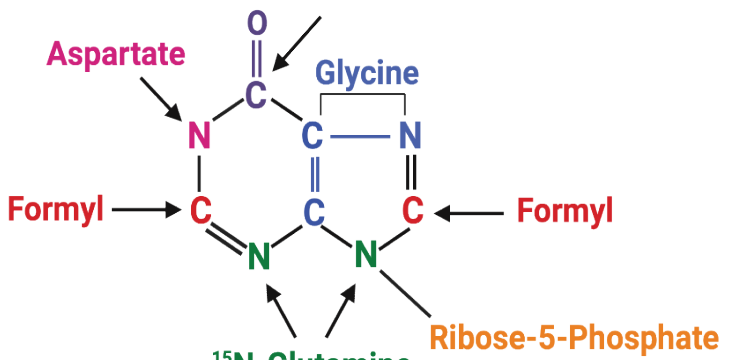

B.

$\left[{ }^{15} \mathrm{~N}_{2}\right]-\mathrm{IMP}$

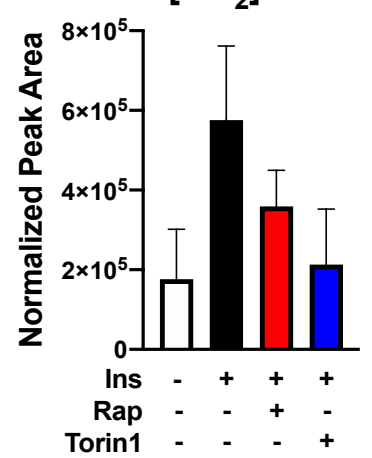

D.

C. RNA-Derived Nucleotides

AMP GMP

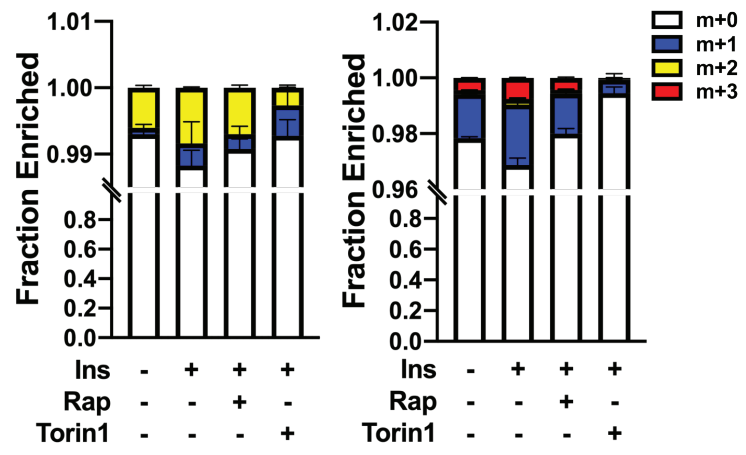

E. RNA-Derived Nucleotides

AMP

GMP
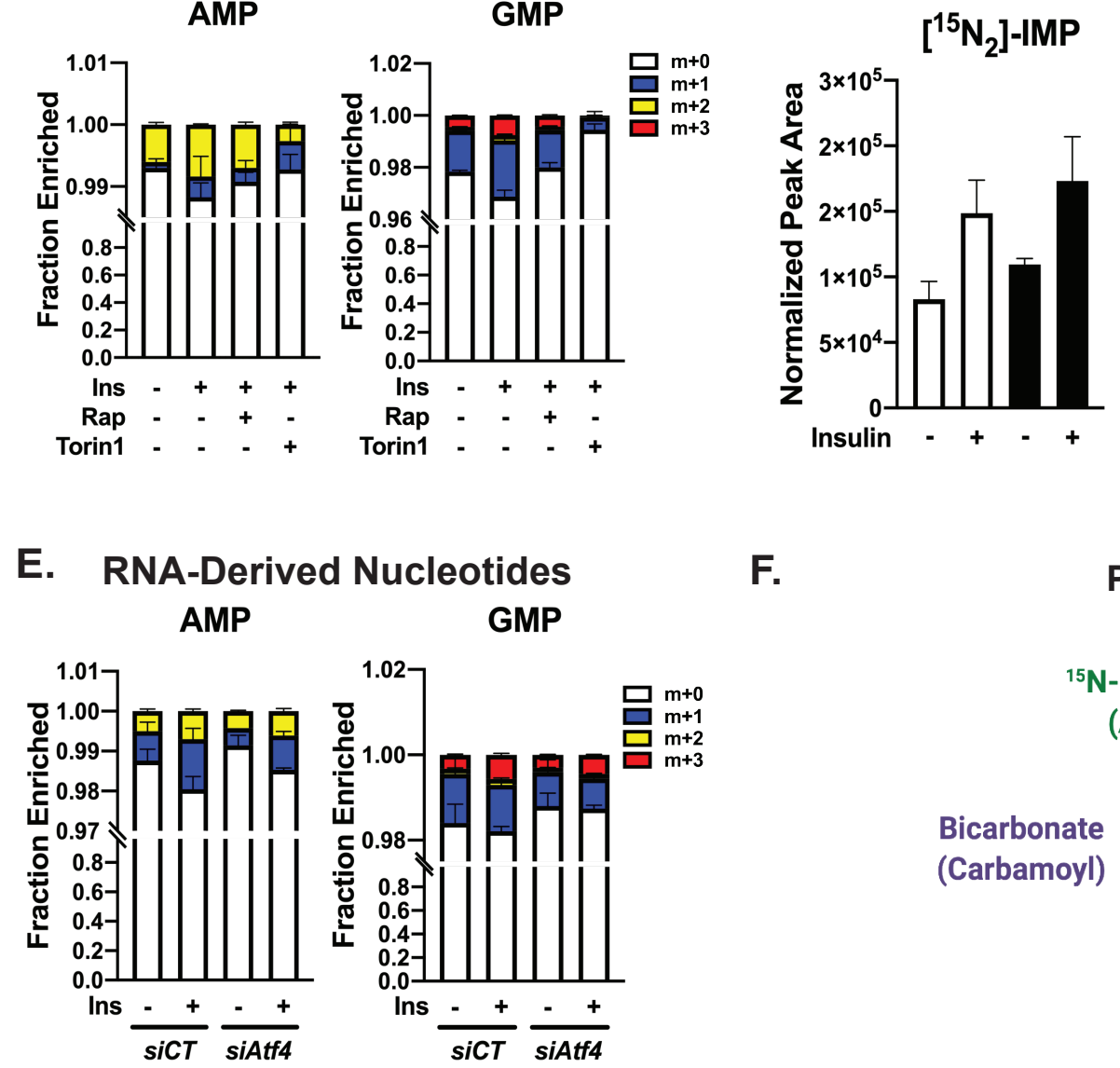

F.
Free Nucleotide Pool
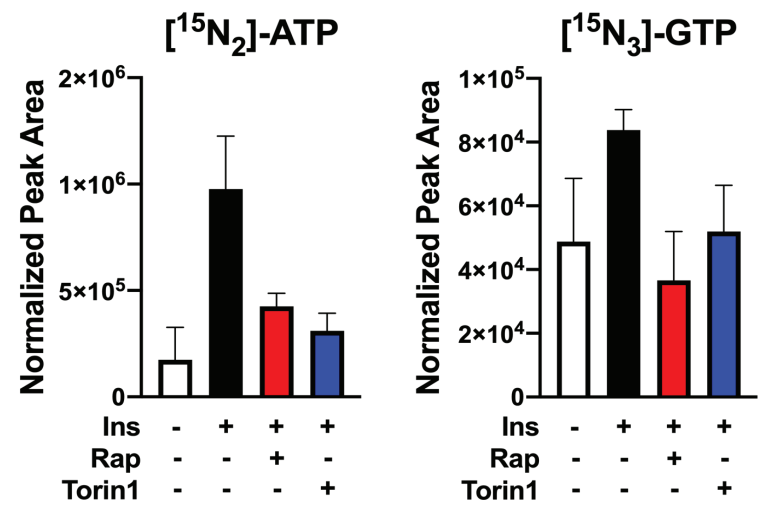

Free Nucleotide Pool
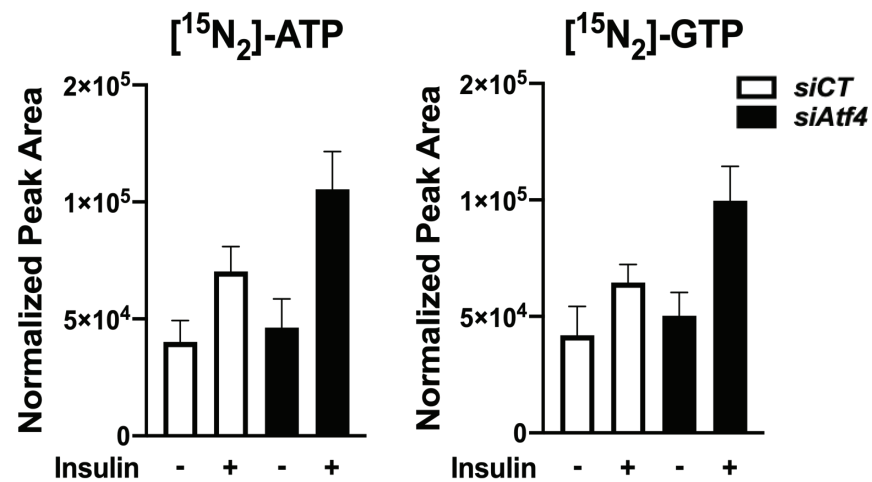

Pyrimidine Nucleotide

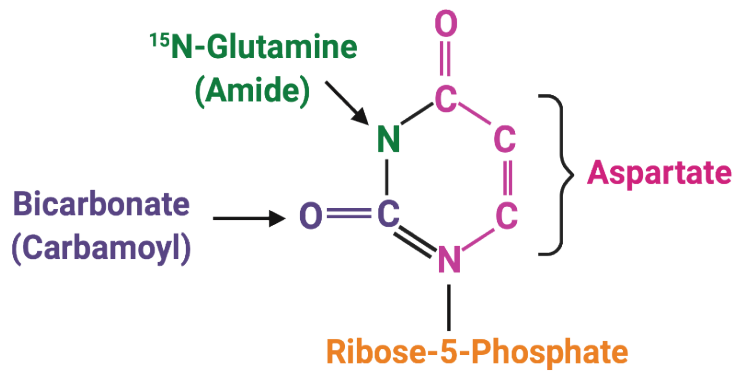

H.

Free Nucleotide Pool

$\left[{ }^{15} \mathrm{~N}_{1}\right]$-UTP

$\left[{ }^{15} \mathrm{~N}_{2}\right]-\mathrm{CTP}$
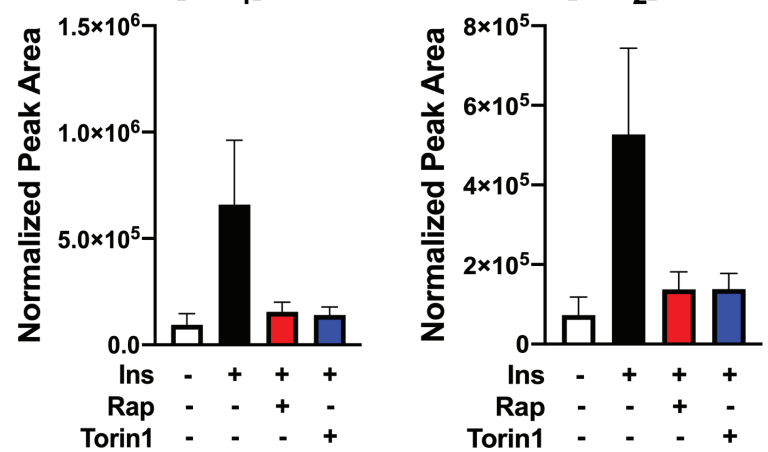

$\left[{ }^{15} \mathrm{~N}_{1}\right]$-UTP
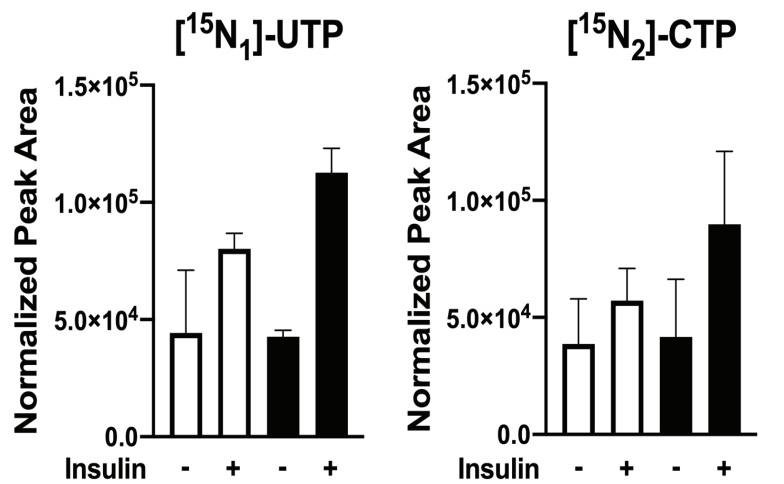

siCT

siAtf4 
Figure 4 Rxiv preprint doi: https://doi.org/10.1101/2021.05.02.442369; this version posted May 3, 2021. The copyright holder for this preprint (which was not certified by peer review) is the author/funder. All rights reserved. No reuse allowed without permission.

A.

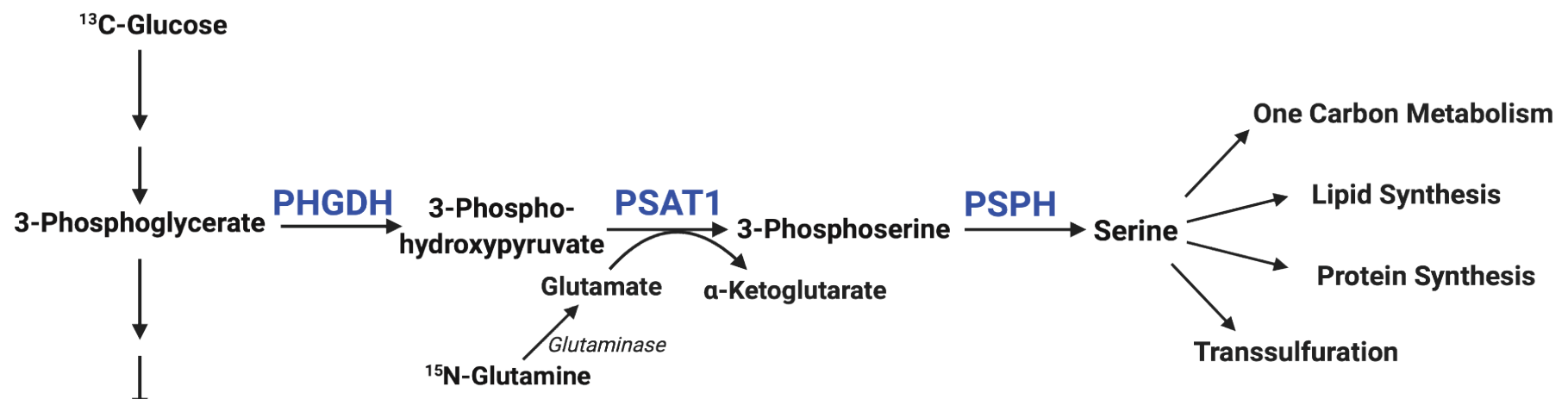

Lactate

\section{${ }^{15} \mathrm{~N}-$ Glutamine-Amine Tracing}

B.

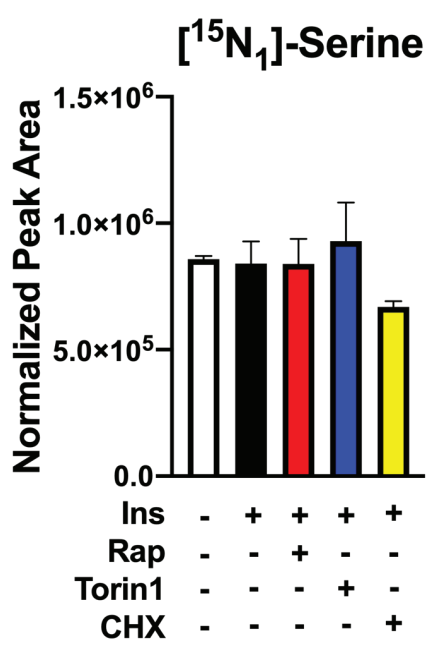

C.

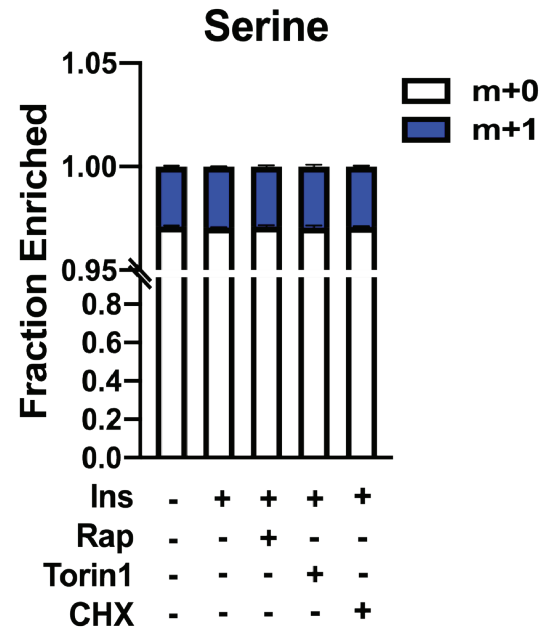

F.

Serine

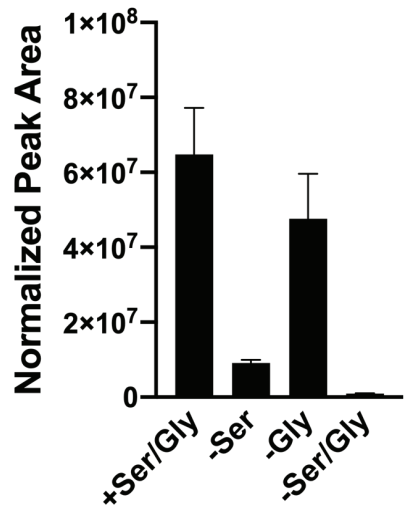

U-13C-Glucose Tracing

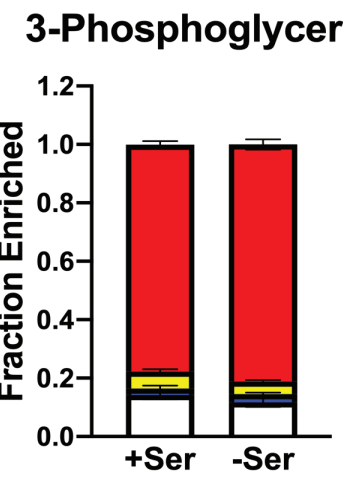

D.

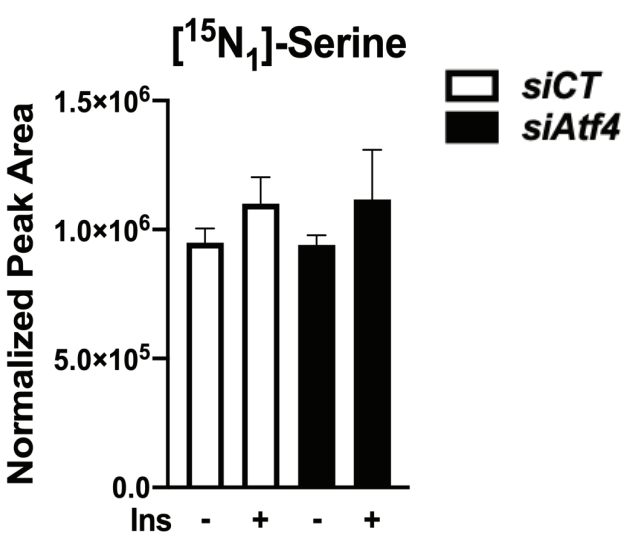

G.

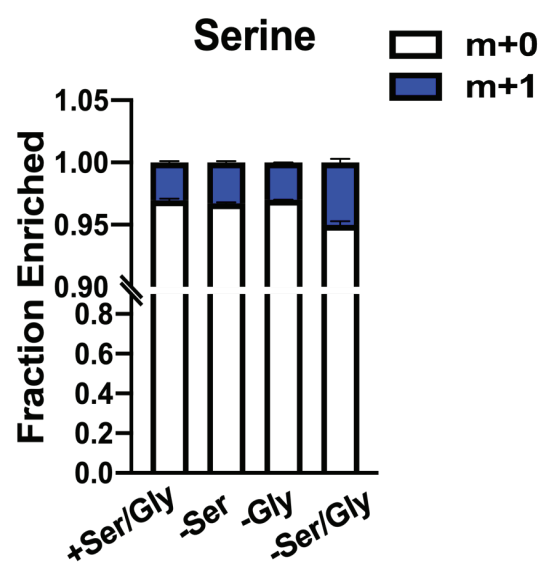

H.

Serine

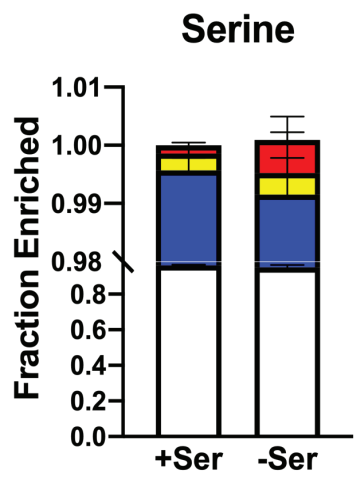

$m+0$

$m+1$

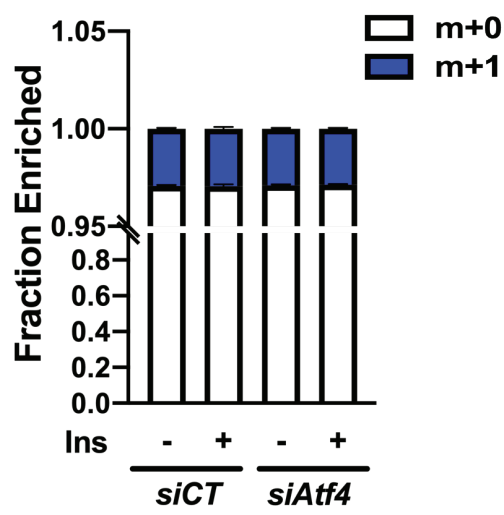

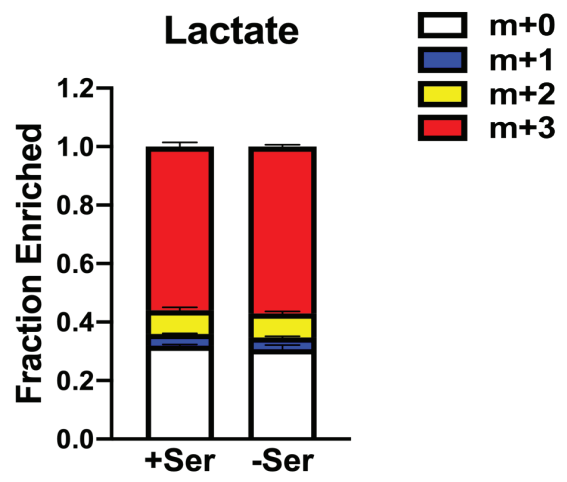



Figure GioRxiv preprint doi: https://doi.org/10.1101/2021.05.02.442369; this version posted May 3, 2021. The copyright holder for this preprint (which
was not certified by peer review) is the author/funder. All rights reserved. No reuse allowed without permission.

A.

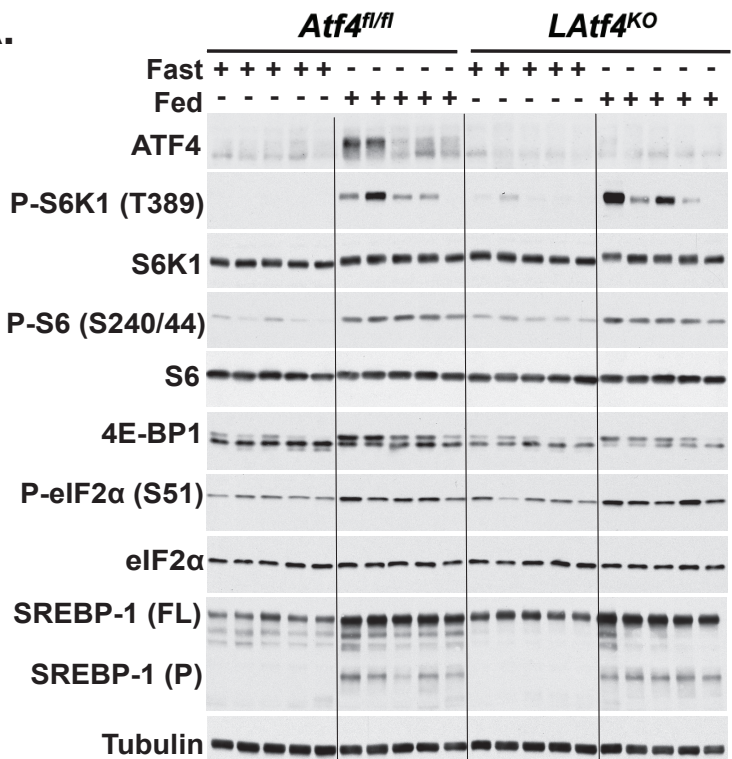

C.

Principal Component Analysis

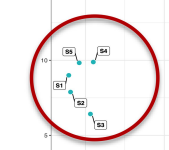

○े Atf4 ${ }^{f / f I}$ Fast

ปั

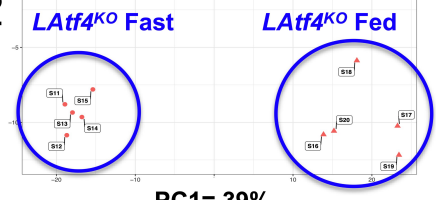

$\mathrm{PC} 1=39 \%$
B.
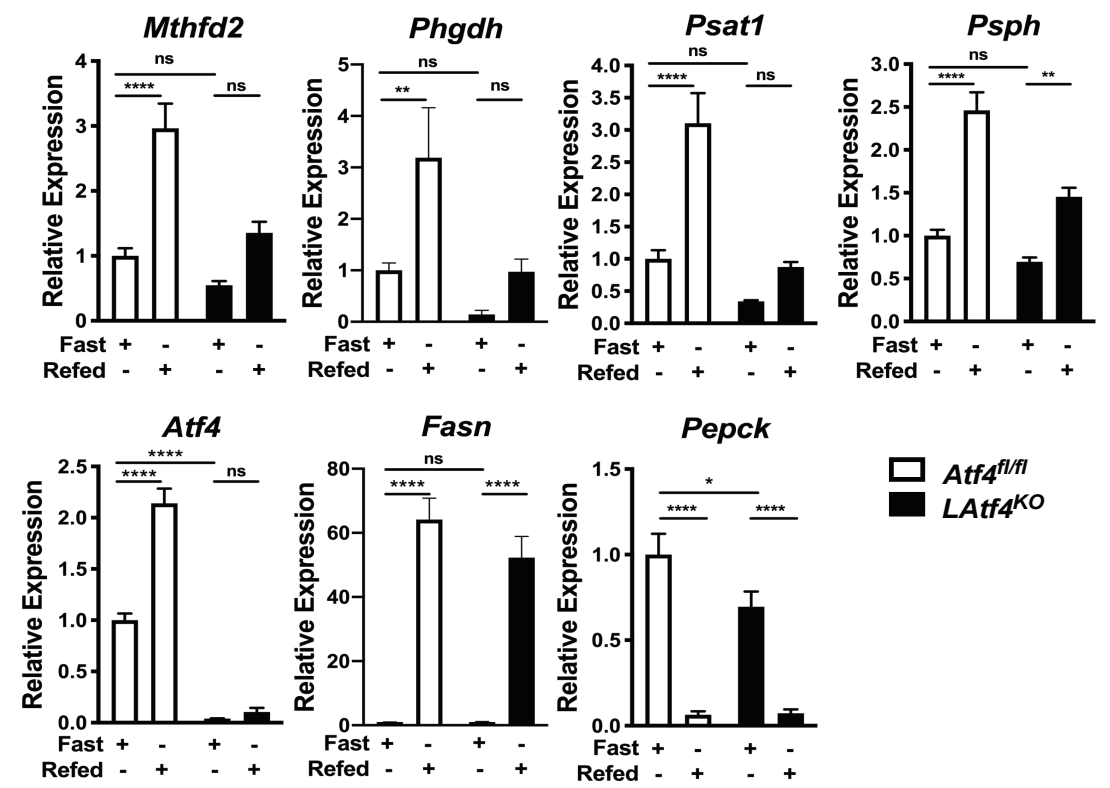

Pepck

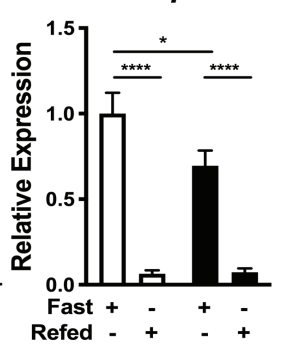

E.

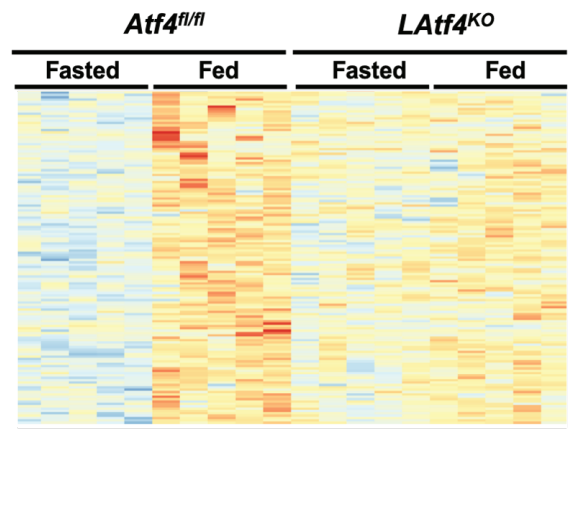

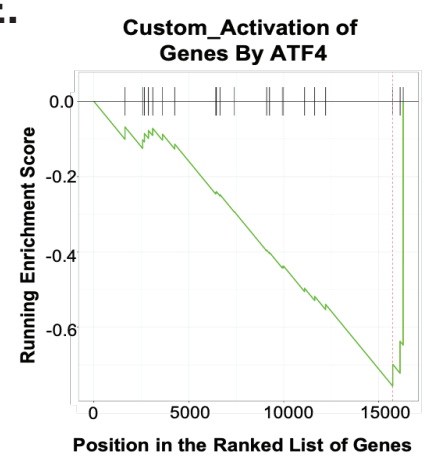

KEGG_Amino Acid Synthesis

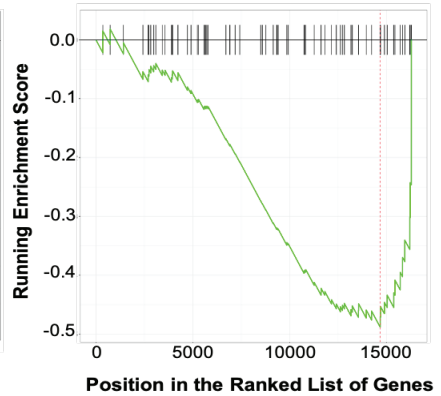

F. LAtf4 ${ }^{K O}$ versus Attf $4^{f / f}$ Fed Livers G.

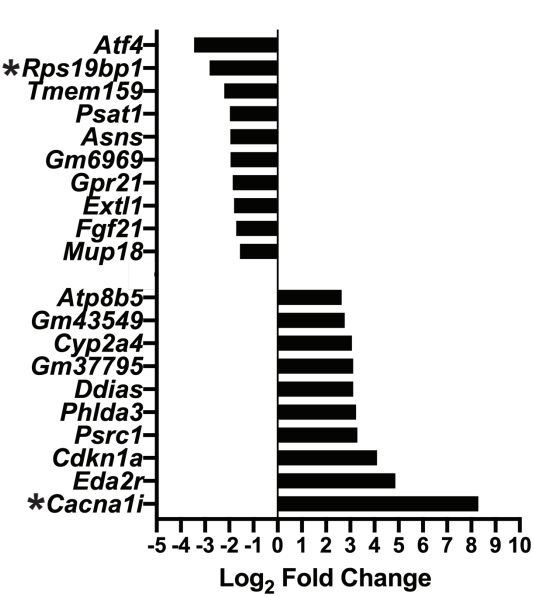

.

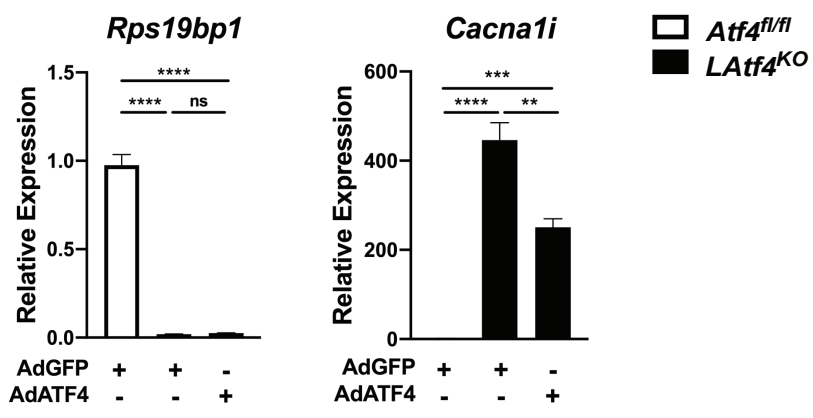

Rps19bp1

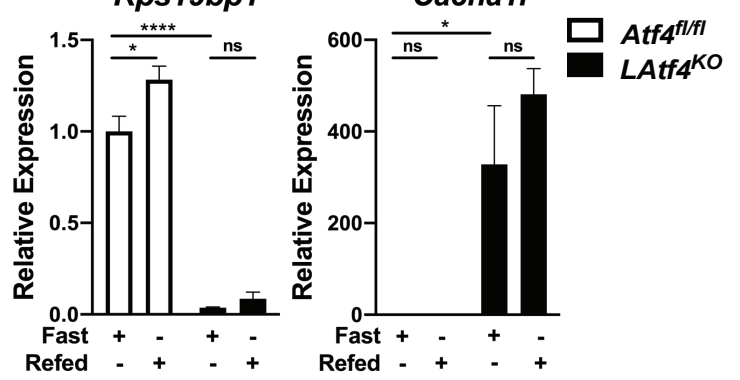

Rps19bp1

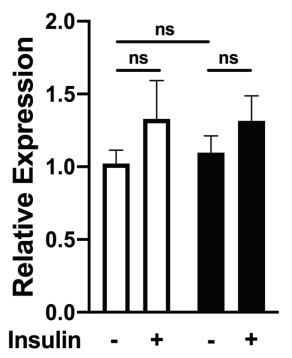

Cacna1i

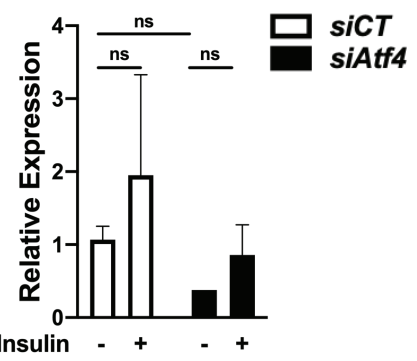

J.

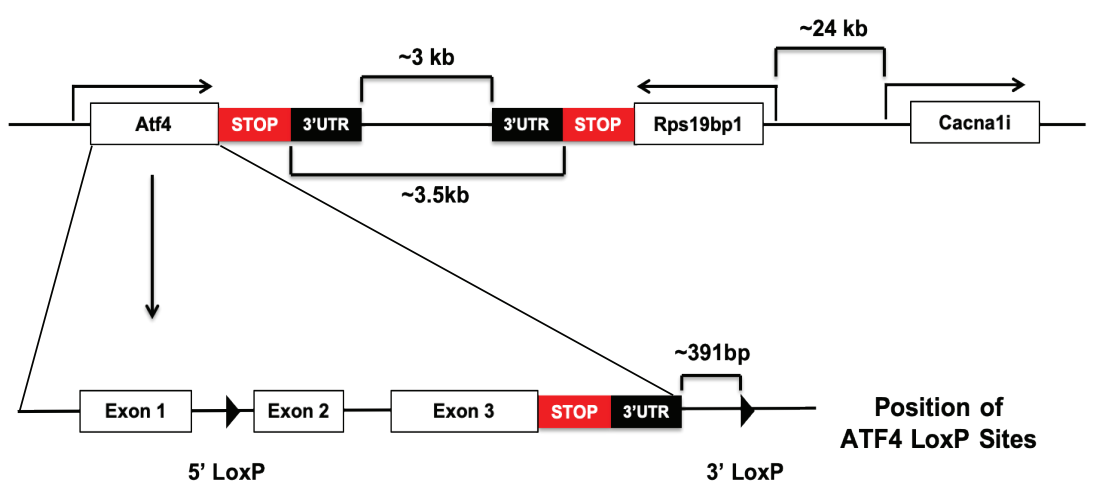


A.

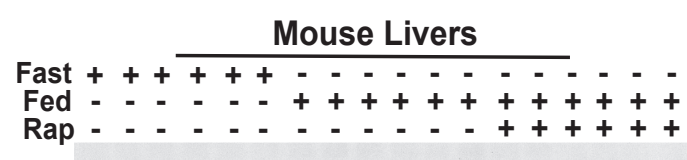

ATF4 (BioLegend)

ATF4 (ProteinTech)

P-S6 (S240/44)

P-S6K1 (T389)

en. $-5-\infty$

S6K1 - - - - - - - - - - -

C.

$\square$ ins

Ins

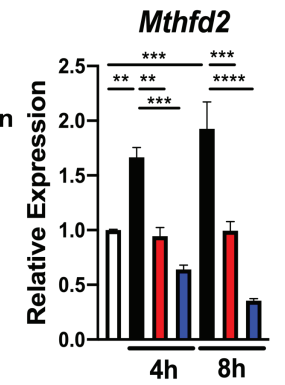

Hepa1-6
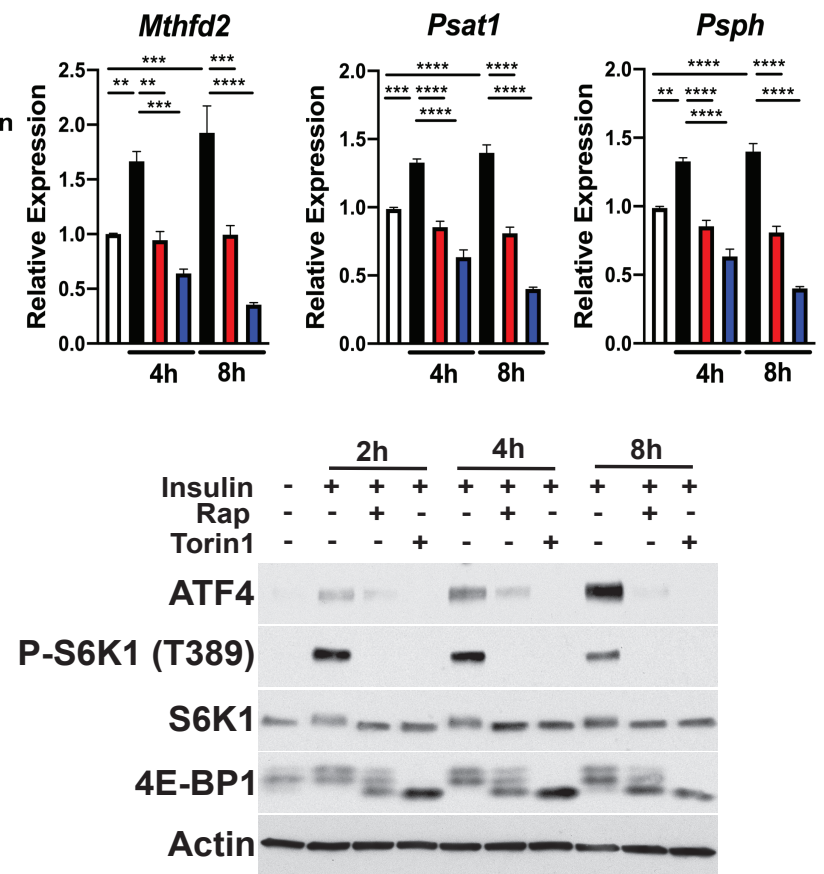

B.

\section{Primary Mouse Hepatocytes}

MTHFD2

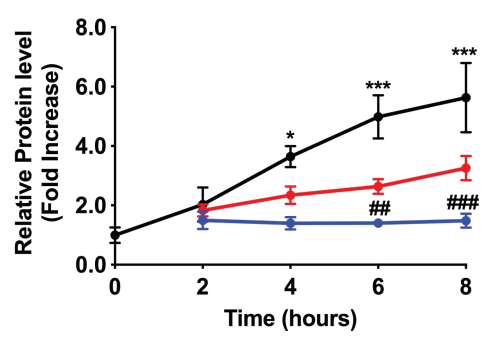

PSAT1
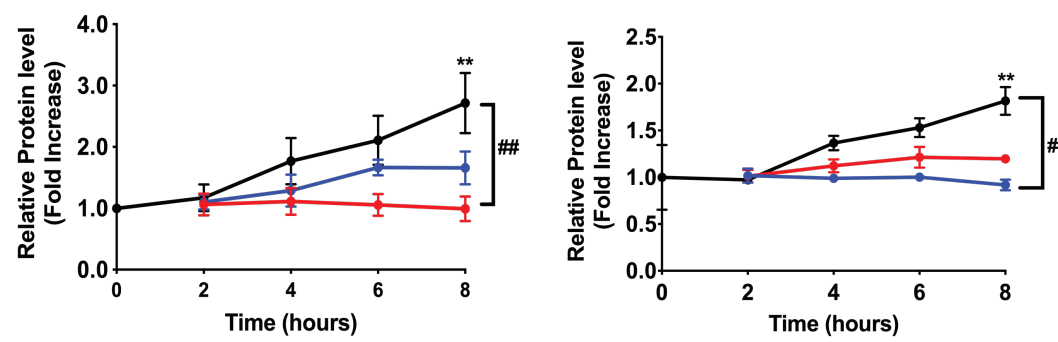

D.
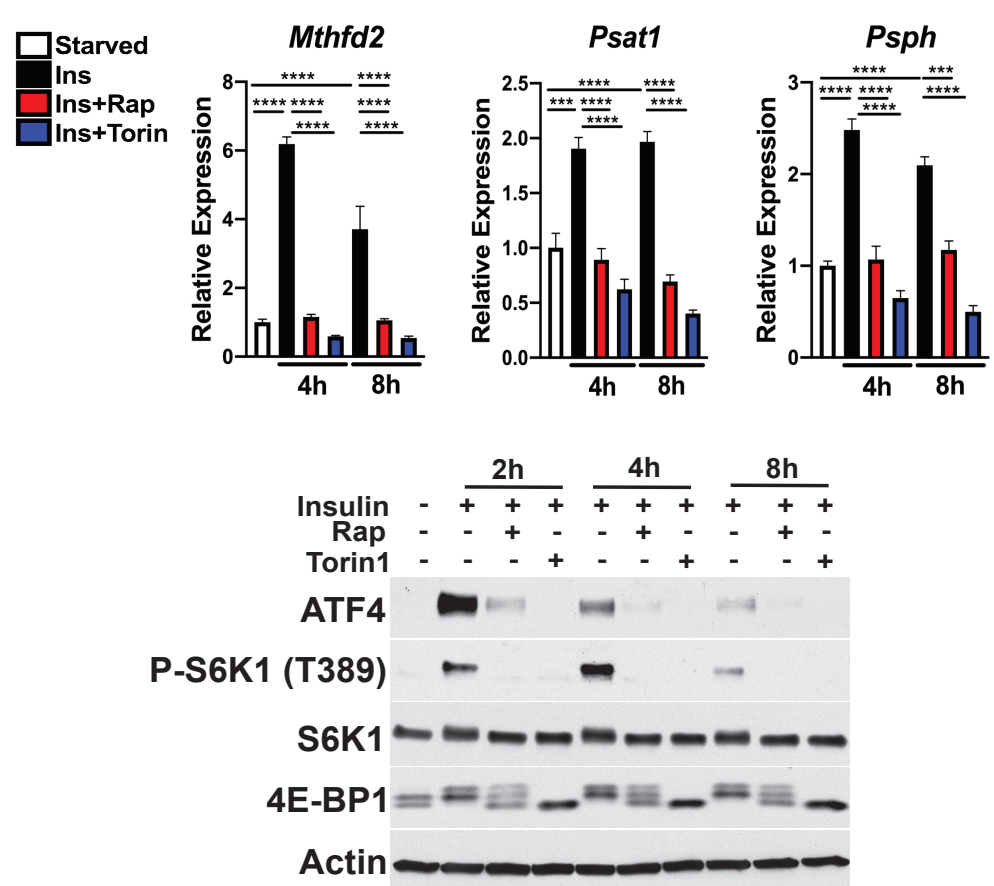

- Insulin

- Insulin+Rap

PHGDH

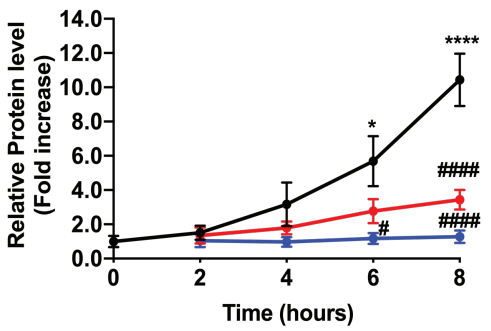

PSPH

$\underline{A M L-12}$

Actin 
A.<smiles>[14CH3][124IH]</smiles>

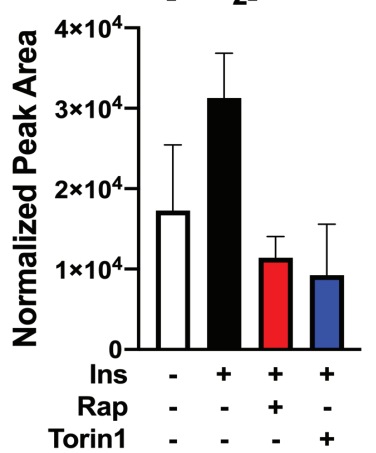

C.

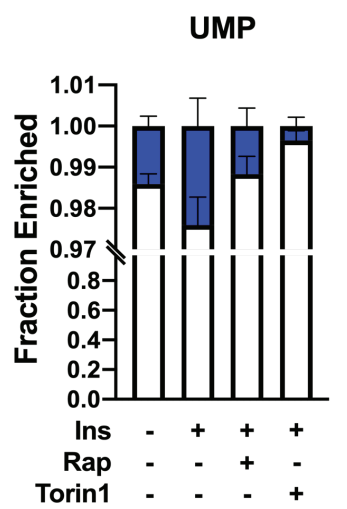

$\left[{ }^{13} C_{2}\right]$-AMP
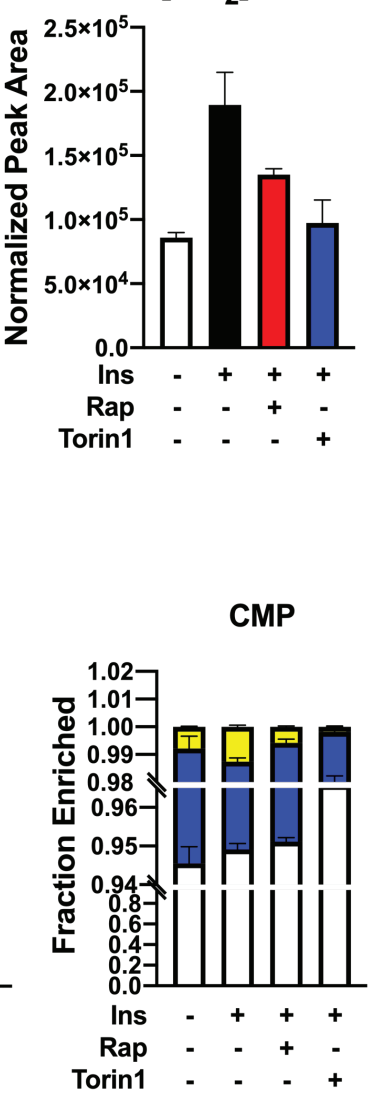

B.
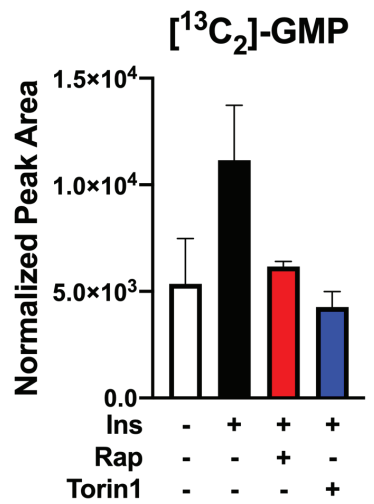

$\left[{ }^{13} \mathrm{C}_{2}\right]$-AMP

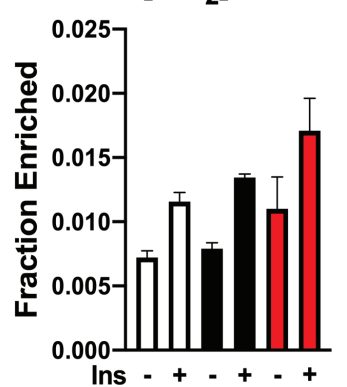

$\left[{ }^{13} \mathrm{C}_{2}\right]$-GMP

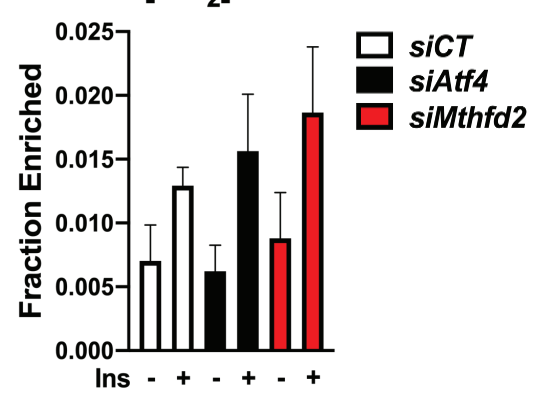

D.

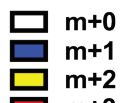

UMP
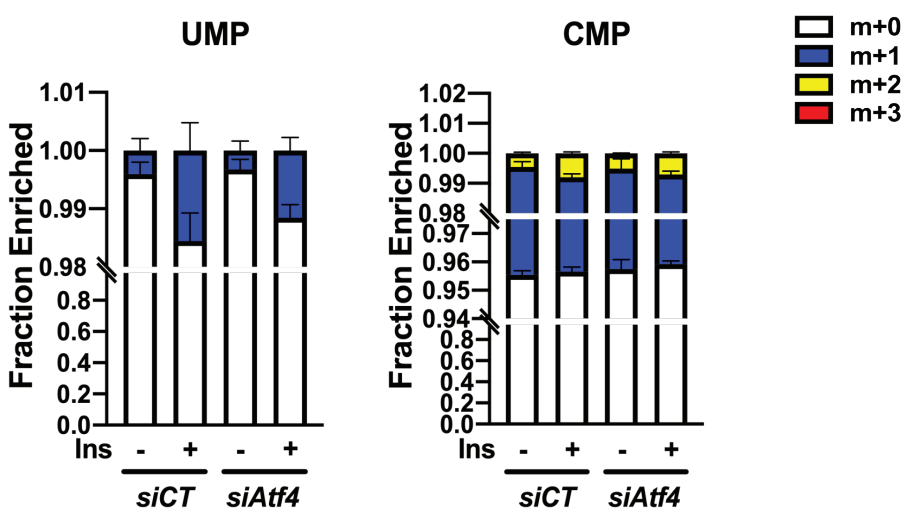
A.

KEGG Metabolite Sets Enrichment (Top 25)

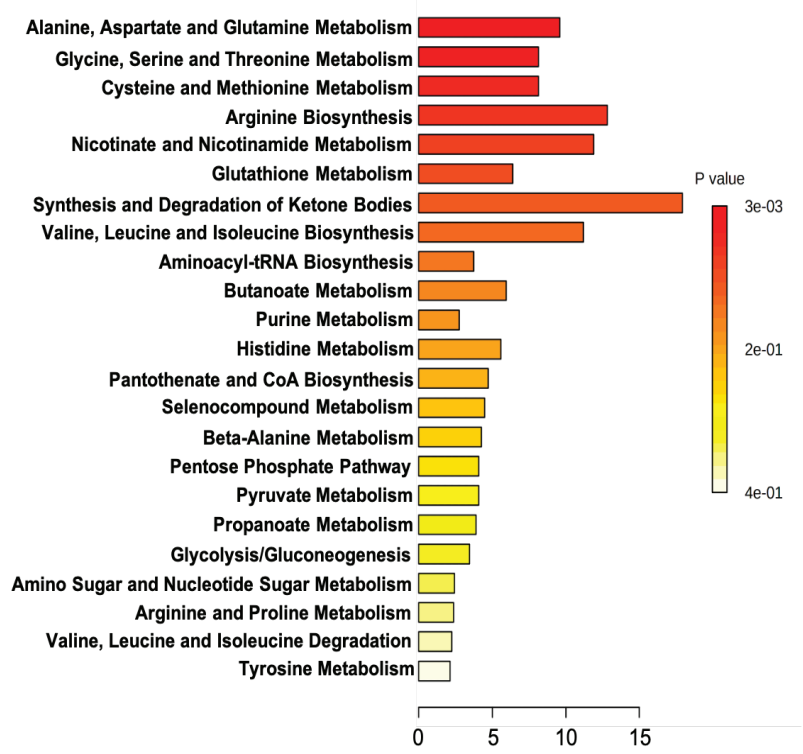

B.

Glutathione

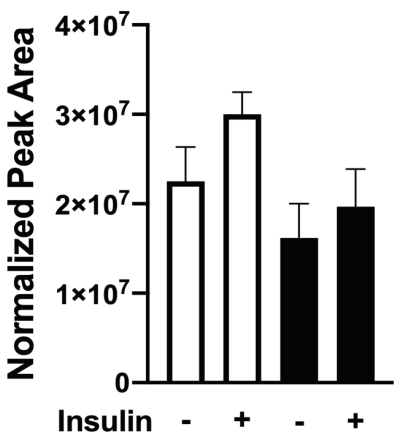

Glutathione Disulfide

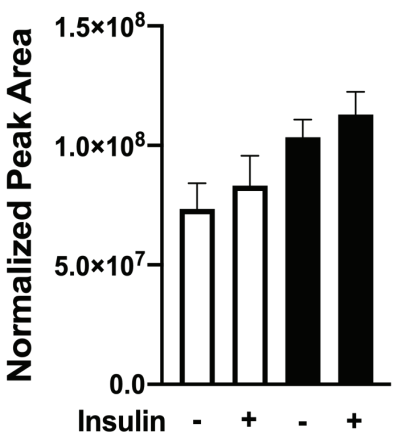

C.

SIc7a11

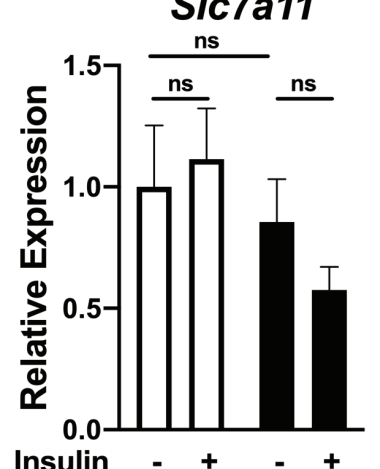

E.

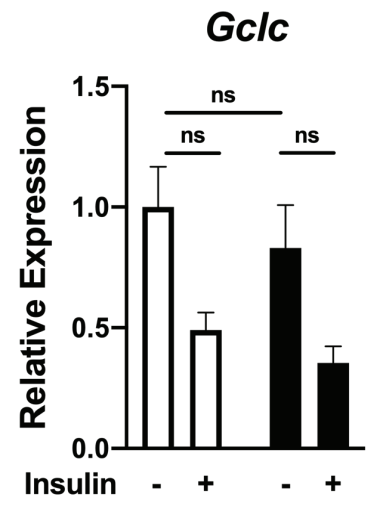

F.

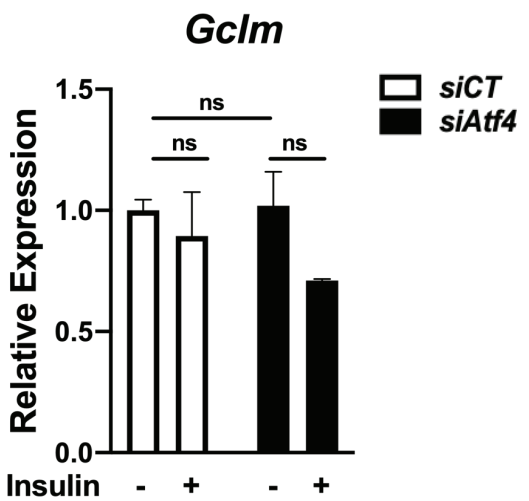

\section{Cth}

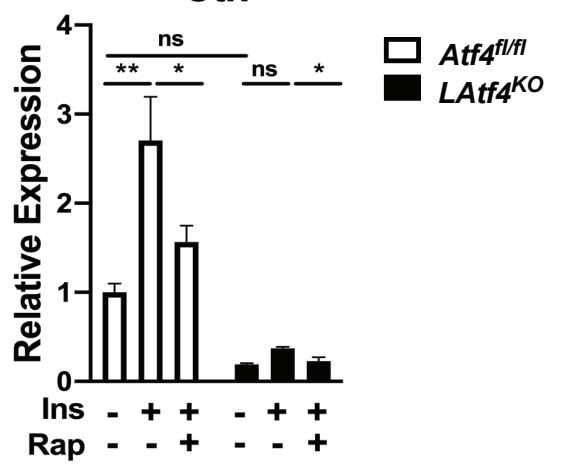

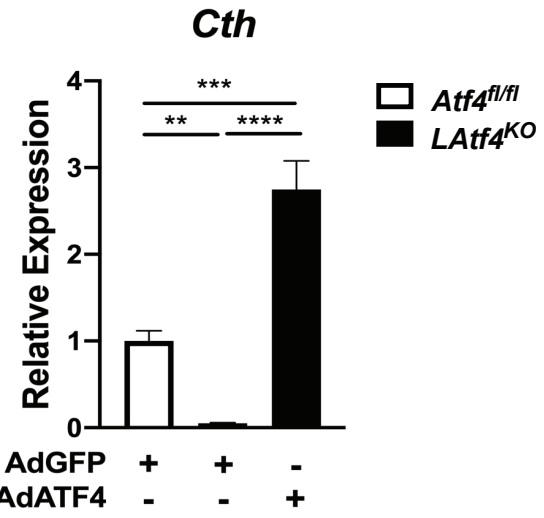

D.

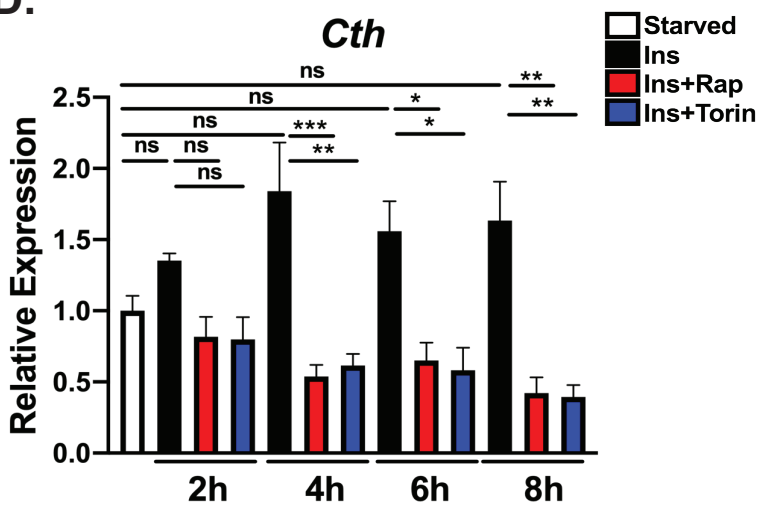

G.

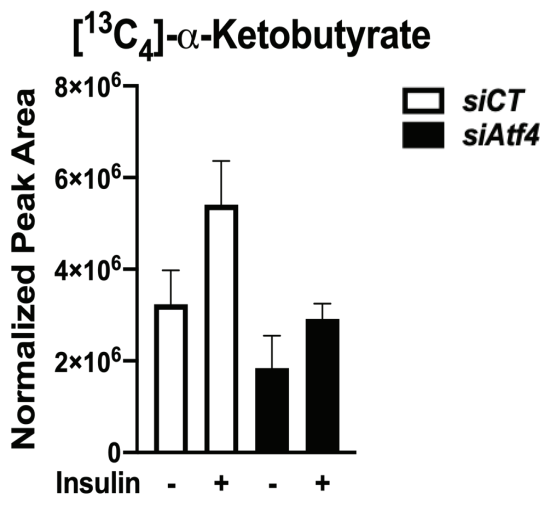


A.

Oxaloacetate $\underset{\text { Glutamate }}{\stackrel{\mathrm{GOT} 1 / 2}{\longrightarrow} \text { A-Ketoglutarate }}$

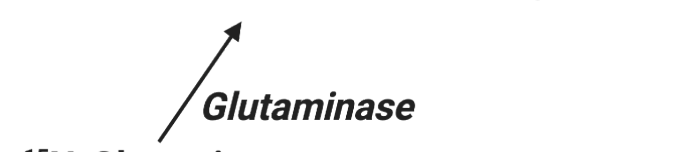

${ }^{15} \mathrm{~N}$-Glutamine
B.

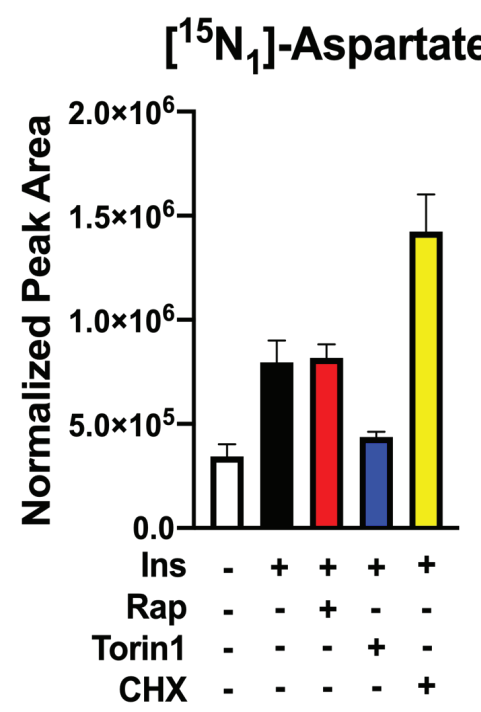

${ }^{15} \mathrm{~N}$-Glutamine-Amine Tracing

D.
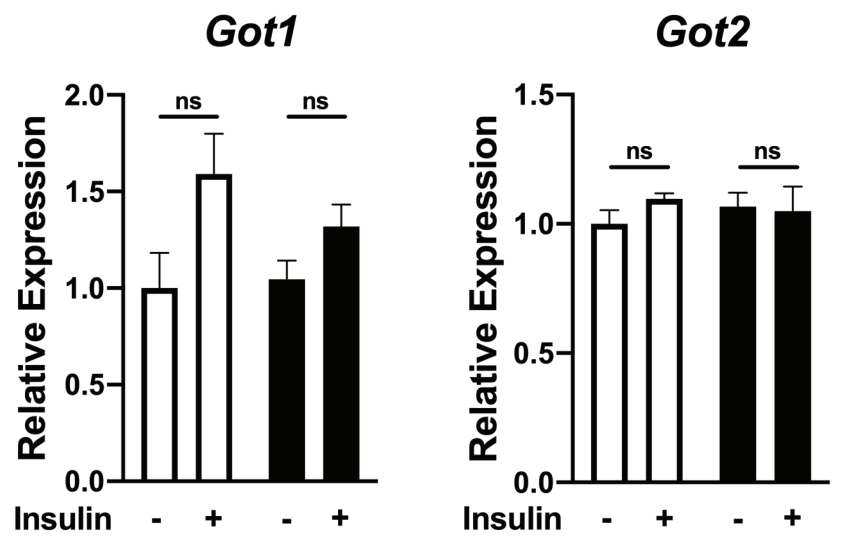

E.

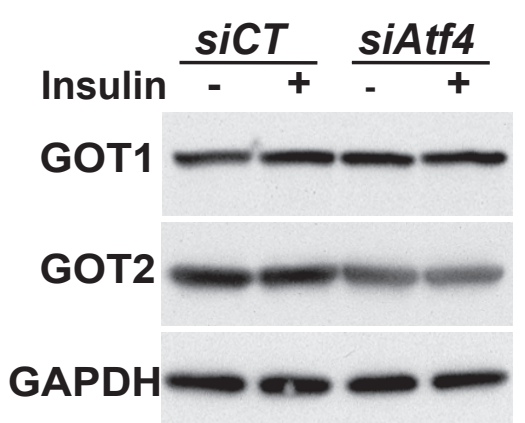

${ }^{15} \mathrm{~N}$-Glutamine-Amine Tracing

F.

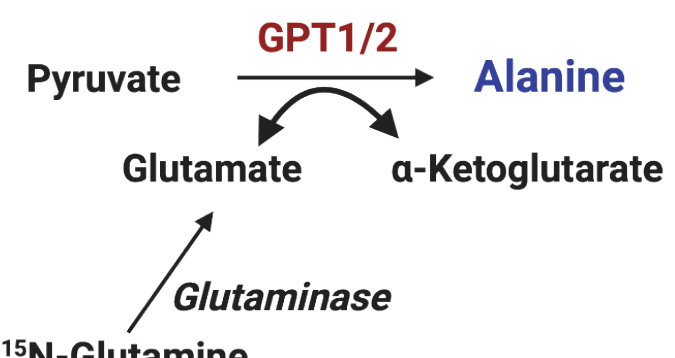

${ }^{15} \mathrm{~N}$-Glutamine

I.

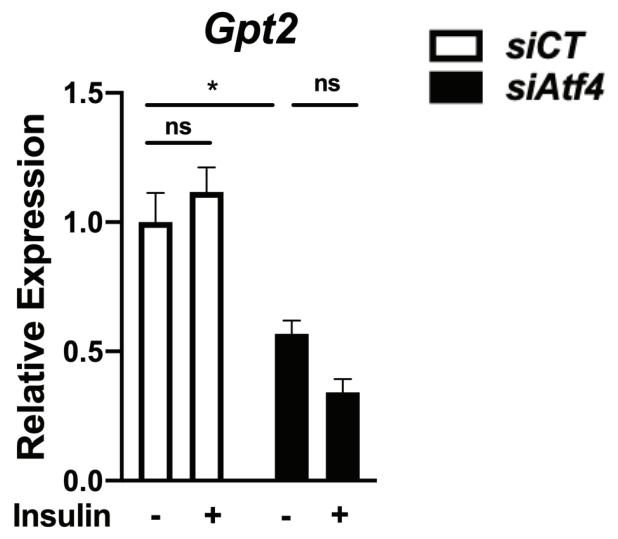

G.

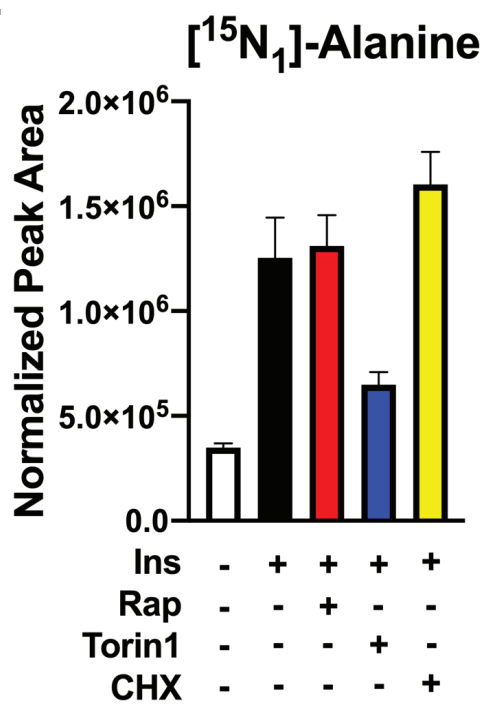

C.

$\left[{ }^{15} \mathrm{~N}_{1}\right]$-Aspartate

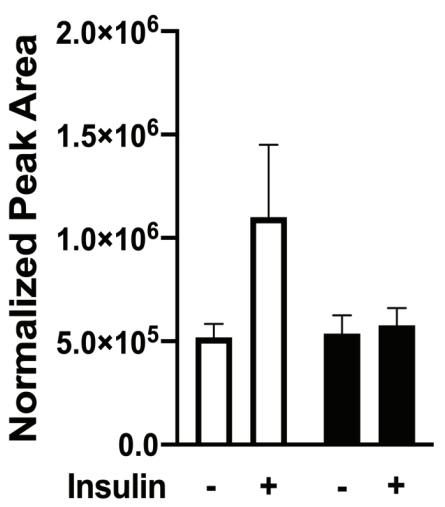

siCT

siAtf4
H.

$\left[{ }^{15} \mathrm{~N}_{1}\right]$-Alanine

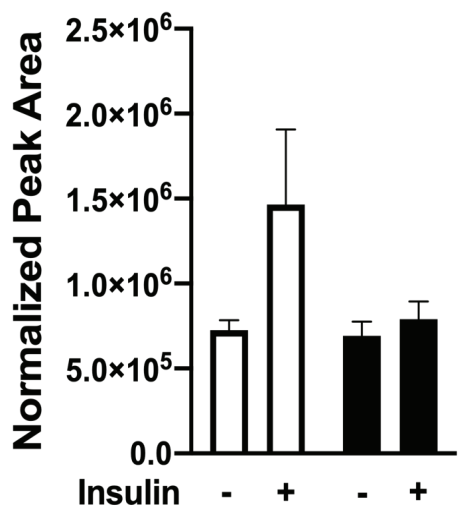




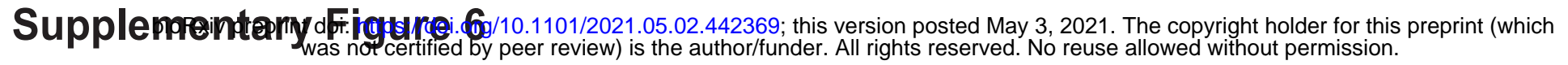

A.

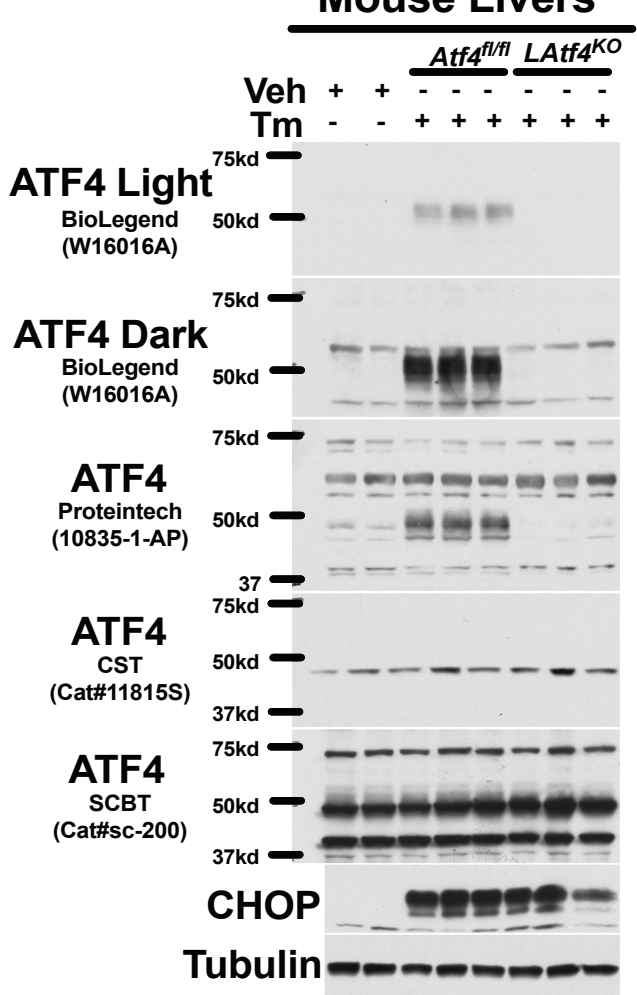

$B$.

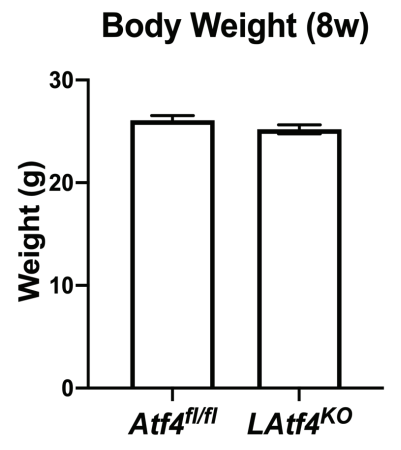

C.

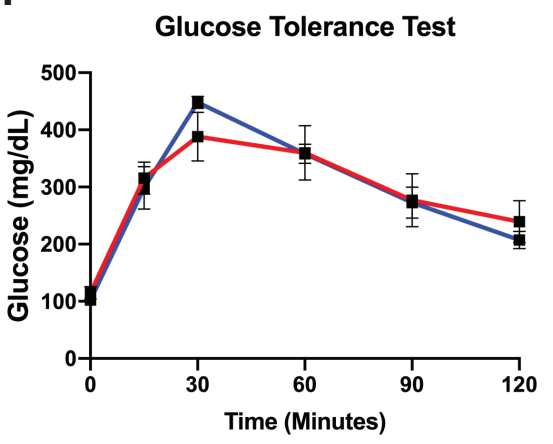

Body Weight (26w)
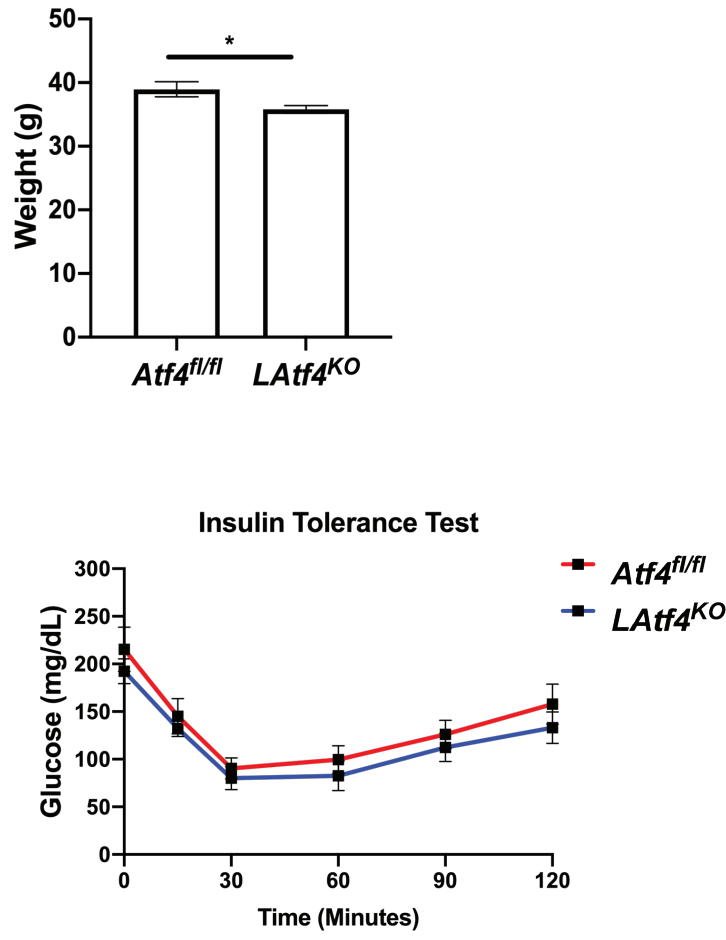
A.

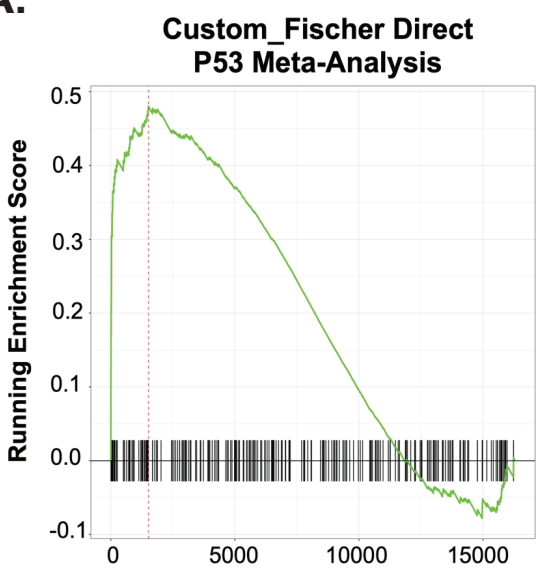

Position in the Ranked List of Genes

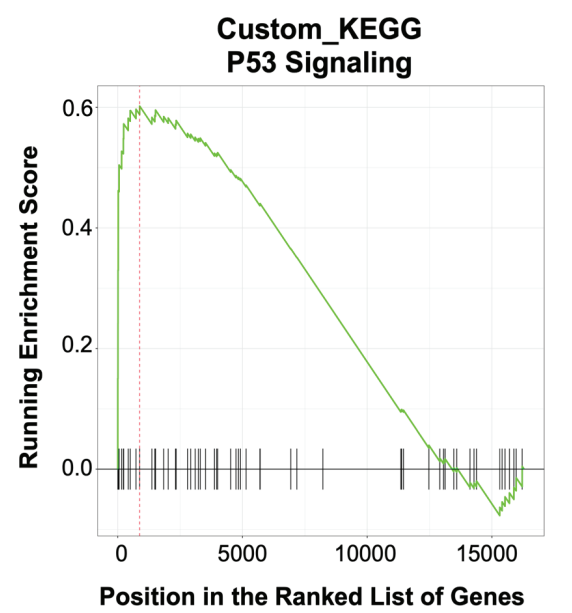

B.

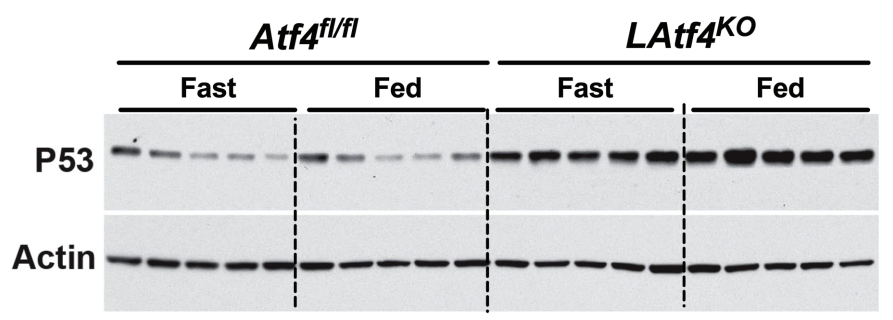

C.

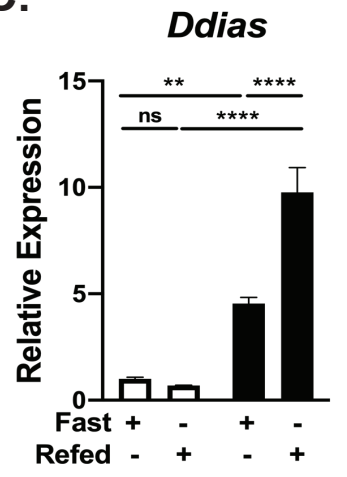

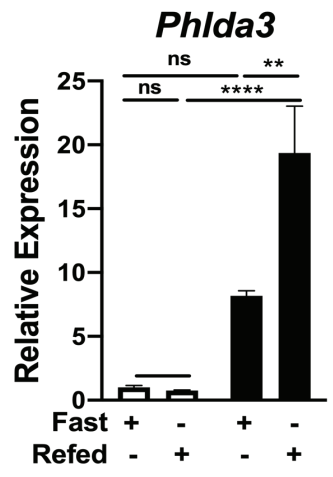

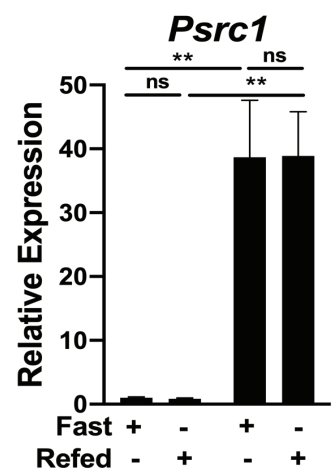

Eda2r

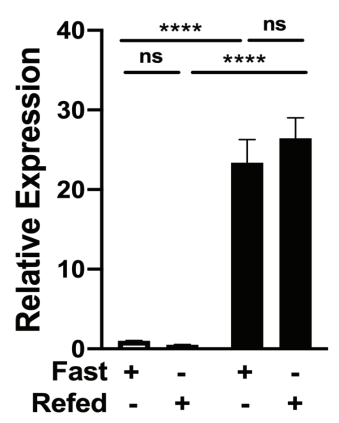

Cdkn1a (p21)

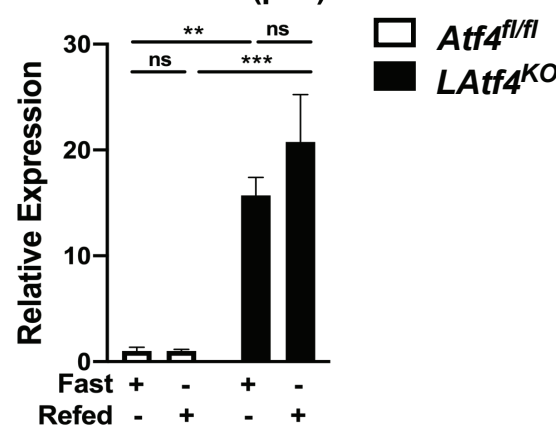

D.

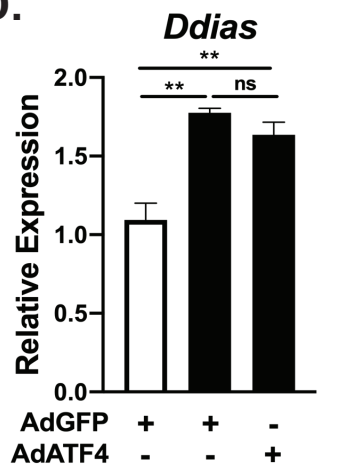

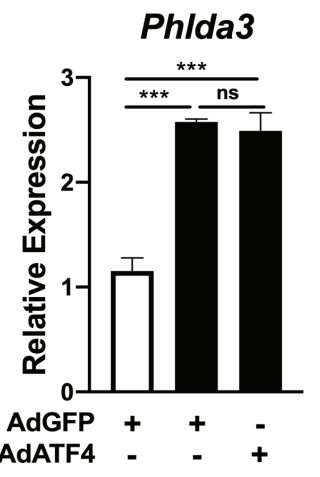
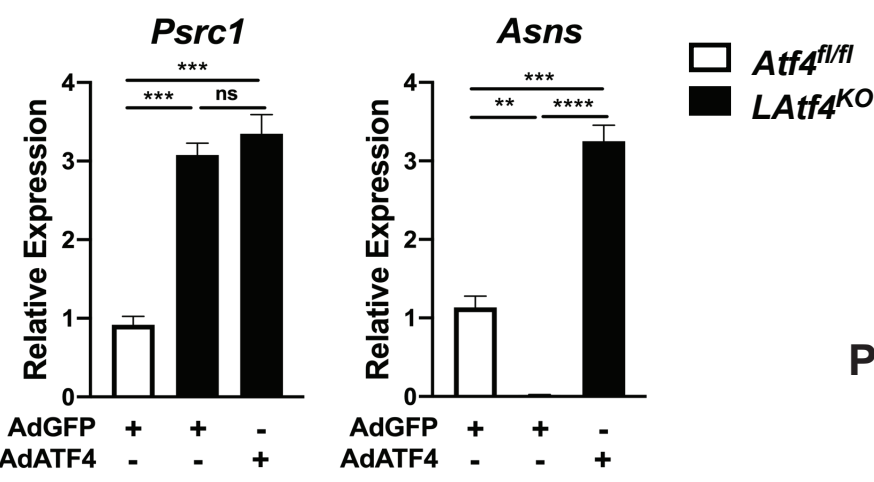

E.

$A t f 4^{f / f I} L A t f 4^{K O}$ AdGFP + + AdATF4 - - +

ATF4

P53

P-elF2 $\alpha$ (S51)

eIF2 $\alpha$

Tubulin

F.

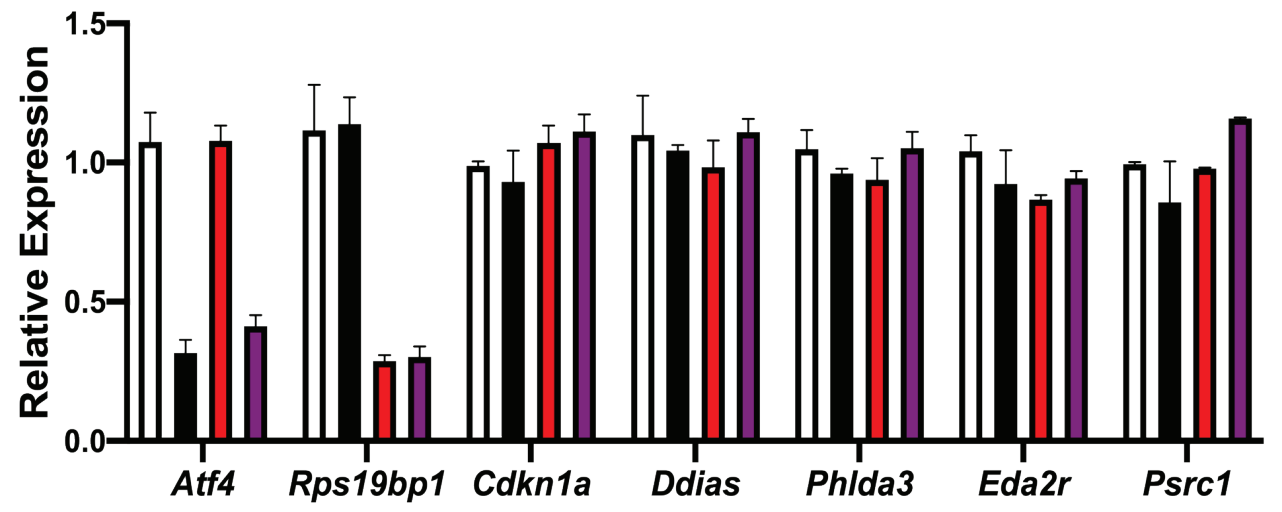

siCT

siAtf4

siRps19bp1

siAtf4+siRps19bp1 